Aus der Abteilung Psychiatrie und Psychotherapie

(Prof. Dr. med. P. Falkai)

im Zentrum Psychosoziale Medizin

der Medizinischen Fakultät der Universität Göttingen

\title{
Vergleichende MR- volumetrische Untersuchung des orbitofrontalen Kortex bei Schizophrenie, bipolarer Störung, Zwangserkrankung und gesunden Kontrollpersonen
}

\author{
INAUGURAL-DISSERTATION \\ zur Erlangung des Doktorgrades \\ der Medizinischen Fakultät \\ der Georg-August-Universität zu Göttingen
}
vorgelegt von
Kristina Meyer
aus

Hannover

Göttingen 2010 
Dekan:

1. Berichterstatter:

2. Berichterstatter:

3. Berichterstatter:
Prof. Dr. med. C. Frömmel

Priv.-Doz. Dr. med. T. Wobrock Priv.-Doz. Dr. rer. nat. Dechent Priv.-Doz. Dr. med. Schmidt

Tag der mündlichen Prüfung: 08. März 2011 



\section{Inhaltsverzeichnis}

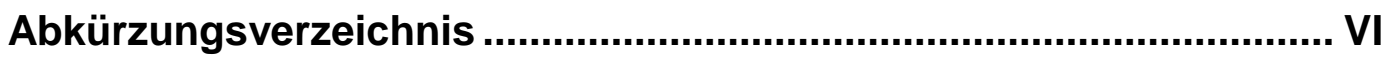

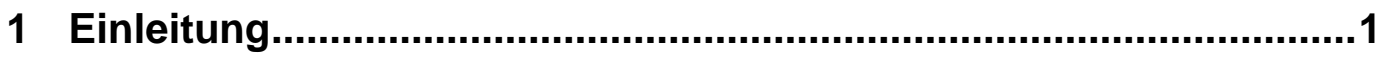

1.1 Neuroanatomie des orbitofrontalen Kortex................................

1.1.1 Projektionen ...........................................................

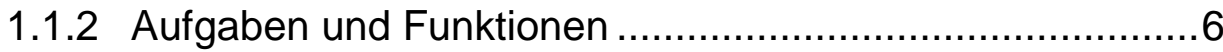

1.2 Der orbitofrontale Kortex im Kontext psychiatrischer

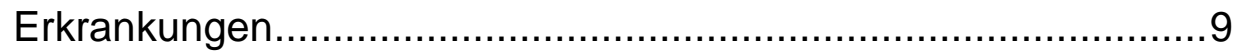

1.2.1 Schizophrenie .........................................................

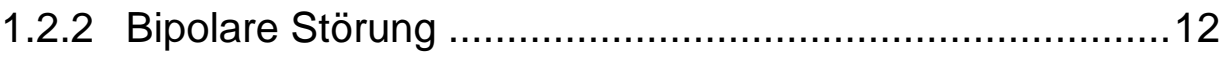

1.2.3 Zwangsstörung ..........................................................13

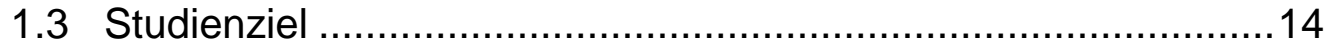

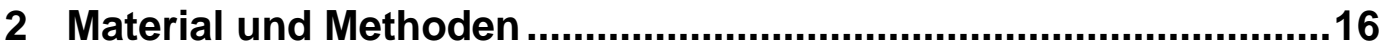

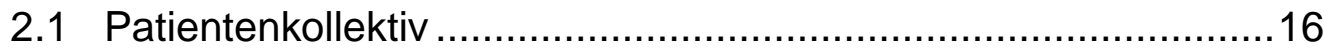

2.2 Beschreibung der psychopathologischen Skalen......................18

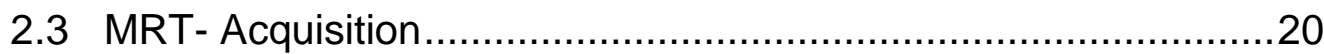

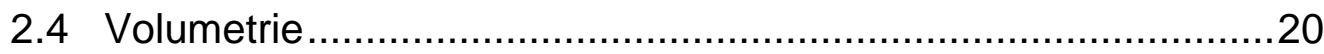

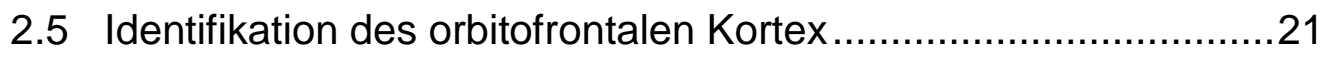

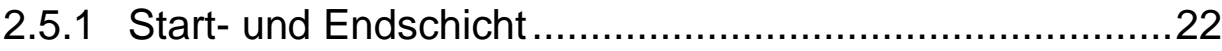

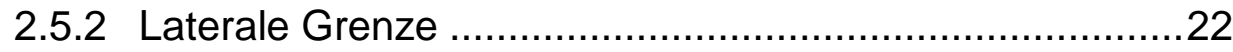

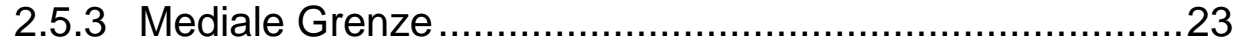

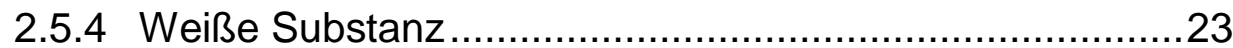

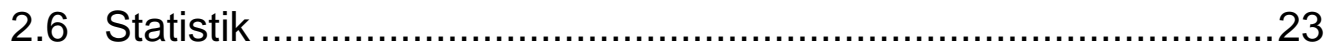

3 Ergebnisse

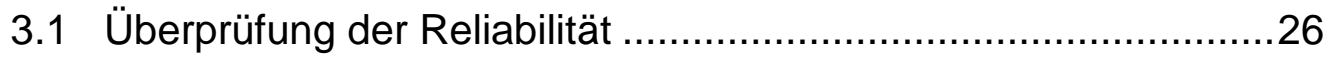

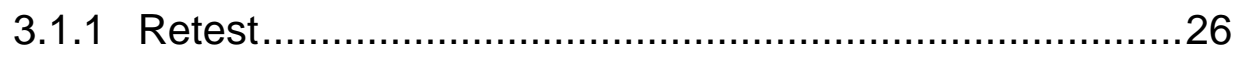

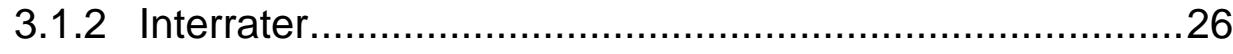

3.2 Vergleich der volumetrischen Größe des orbitofrontalen Kortex bei den verschiedenen Patientengruppen ...................................27

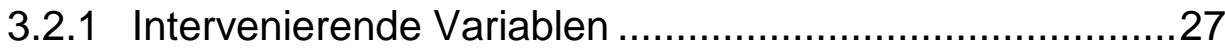

3.2.2 Subgruppenanalyse .................................................. 31 
3.2.2.1 Schizophrenie ........................................... 31

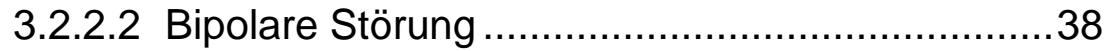

3.2.2.3 Zwangsstörung .....................................43

3.2.3 Diagnose - übergreifende Analysen .........................47

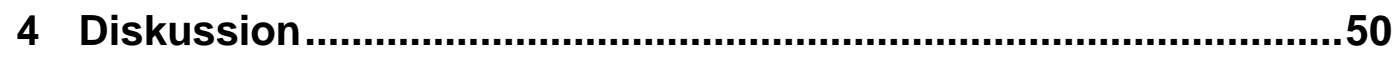

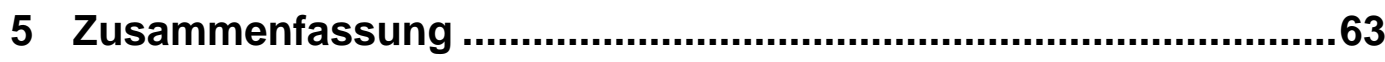

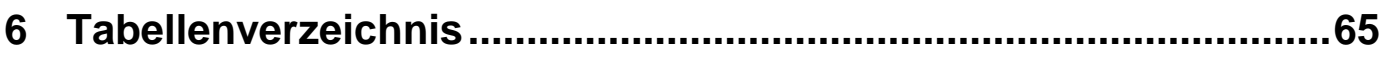

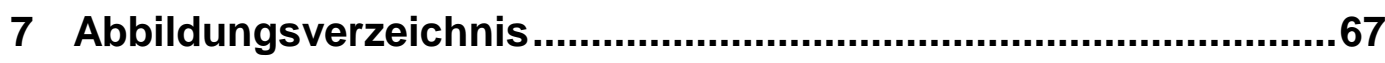

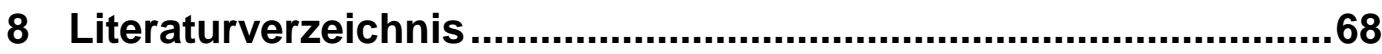

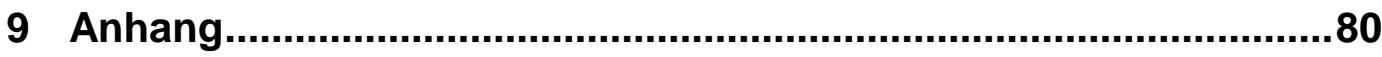

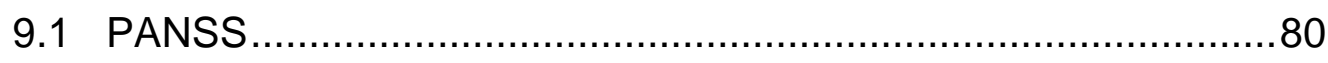

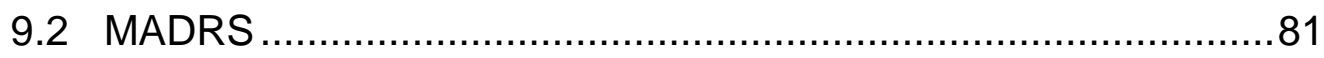

9.3 CGI (Clinical Global Impressions) .................................83

9.4 GAF (Global Assessment of Functioning) ......................... 84 


\section{Abkürzungsverzeichnis}

\begin{tabular}{|c|c|}
\hline$A B$ & Abitur \\
\hline ANCOVA & Analysis Of Covariance \\
\hline ANOVA & Analysis Of Variance \\
\hline Asymm. - Koeff. & Asymmetrie - Koeffizient \\
\hline $\mathrm{Bcl}-2$ & B- Zell Lymphom Protein 2 \\
\hline CGI & Clinical Global Impressions \\
\hline CPZ & Chlorpromazin \\
\hline CSI & Circular insular Sulcus \\
\hline $\mathrm{df}$ & Freiheitsgrade \\
\hline DSM-IV & $\begin{array}{l}\text { Diagnostisches und Statistisches Manual Psychischer } \\
\text { Störungen, vierte Revision }\end{array}$ \\
\hline $\mathrm{F}$ & F- Statsitik \\
\hline FMS & Frontomarginaler Sulcus \\
\hline GAF & Global Assessment of Functioning \\
\hline GFAP & Saures Gliafaserprotein (glial fibrillary acidic protein) \\
\hline GS & Graue Substanz \\
\hline GSK-3 $\beta$ & Glykogen Synthase Kinase- $3 \beta$ \\
\hline HS & Hauptschule \\
\hline ICC & Intraklassen-Korrelationskoeffizient \\
\hline ICD-10 & $\begin{array}{l}\text { International Statistical Classification of Diseases and Related } \\
\text { Health Problems, zehnte Auflage }\end{array}$ \\
\hline LOS & Sulcus orbitalis lateralis \\
\hline MADRS & Montgomery-Asberg depression rating scale \\
\hline MANCOVA & Multivariate Analysis of Covariance \\
\hline MMSE & Mini Mental State Examination \\
\hline MPRAGE & Magnetization Prepared Rapid Gradient Echo \\
\hline MRT & Magnetresonanztomographie \\
\hline OCD & Obsessive Compulsive Disorder \\
\hline OFC & Orbitofrontaler Kortex \\
\hline
\end{tabular}




\begin{tabular}{|l|l|}
\hline$p$ & Fehlerwahrscheinlichkeit der ersten Art \\
\hline$p . c$. & Partielle Korrelation \\
\hline PANSS & Positive And Negative Syndrome Scale \\
\hline PCM 1 & Pericentriolar Material 1 \\
\hline PFC & Präfrontaler Kortex \\
\hline ROI & Region of Interest \\
\hline RS & Realschule \\
\hline SRS & Sulcus rostralis superior \\
\hline ST & Studium \\
\hline TGS & Totale graue Substanz \\
\hline TWS & Totale weiße Substanz \\
\hline VS. & versus \\
\hline WS & Weiße Substanz \\
\hline YMRS & Young Mania Rating Scale \\
\hline
\end{tabular}




\section{$1 \quad$ Einleitung}

\subsection{Neuroanatomie des orbitofrontalen Kortex}

Der orbitofrontale Kortex nimmt im Primatenhirn die ventrale Seite des Frontallappens ein und wird zum Präfrontalhirn gezählt. Von den anderen Teilen des Präfrontalhirns kann er dadurch abgegrenzt werden, dass er als einziger Projektionen der Pars magnocellularis des Nucleus mediodorsalis thalamici erhält (Fuster 1980).

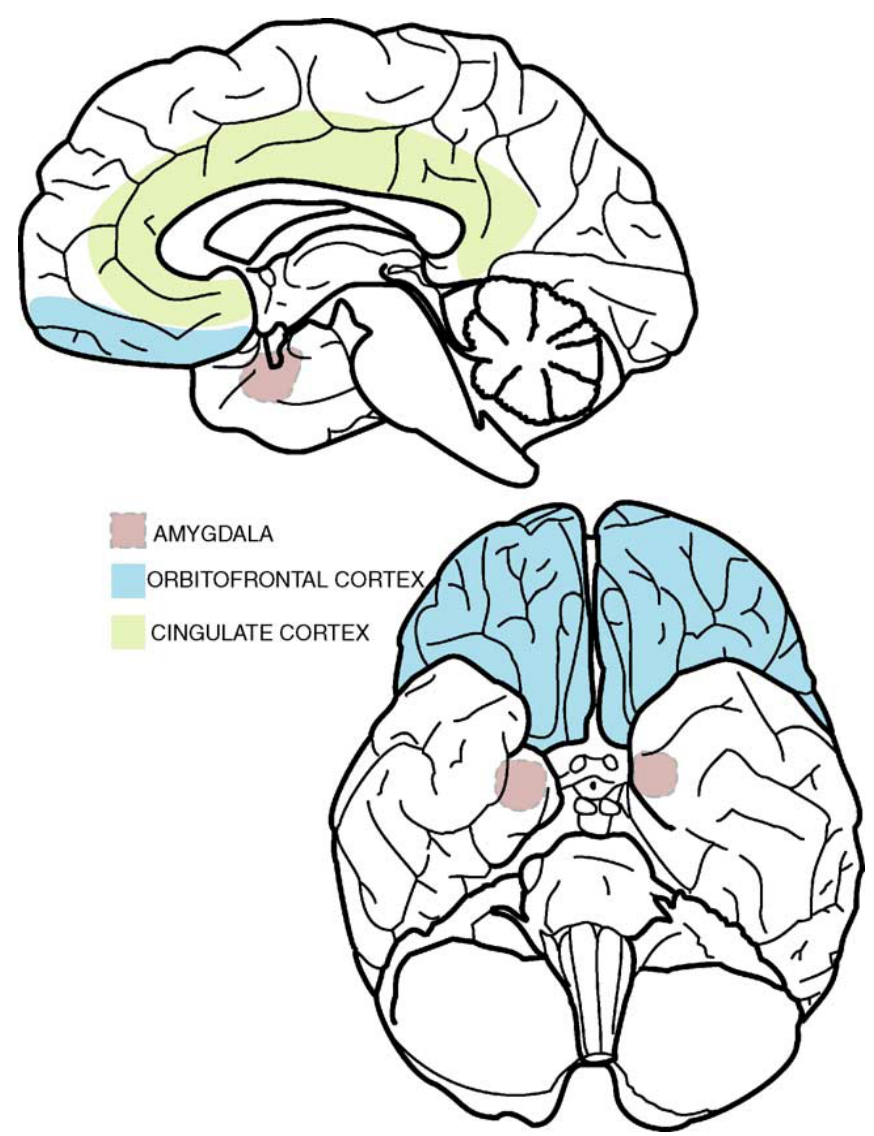

Abbildung 1: Lage des orbitofrontalen Kortex (Kringelbach und Rolls 2004, S.343)

Nach Analyse des Cercopithecus Affen erstellte Brodmann (1909) als Erster eine anatomische Einteilung des präfrontalen Kortex und gliederte den orbitofrontalen Kortex in die Areale 10, 11 und 47. Diese Unterteilung war jedoch nicht zufriedenstellend, vor allem, weil sie sich nicht problemlos auf das menschliche Gehirn übertragen ließ. Weitere Klarheit schuf Walker mit der Untersuchung der Affenspezies Macaca fascicularis (Walker 1940). Er schlug eine differenziertere 
Gliederung in die veränderten Areale 10, 11, 12, 13 und 14 vor. Walkers Area 12 bildet dabei die laterale, Area 13 die mediale Oberfläche. Area 14 liegt an der konvexen Grenze zum Gyrus rectus, der Frontalpol beherbergt Area 10 und als Area 11 wird der restliche anteriore Bereich bezeichnet. Der Kritikpunkt an dieser Einteilung war, dass die humane Area 47 in der neuen Einteilung keine Erwähnung fand. Petrides und Pandya (2002) brachten die humane und nichthumane Karte schließlich in Einklang, indem sie den lateralen Teil der orbitofrontalen Gyri als Area 47/12 bezeichneten (Siehe Abbildung 2).
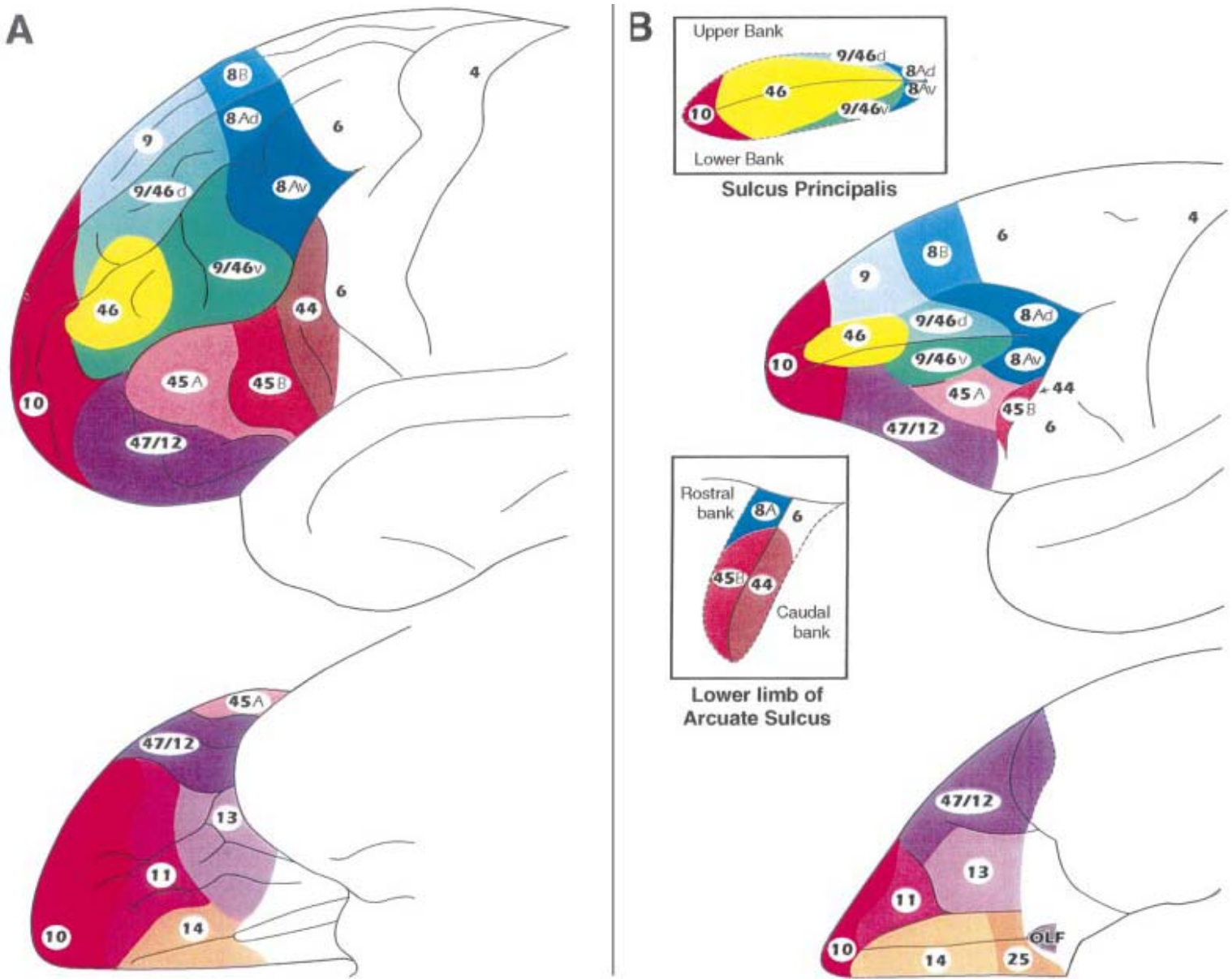

Abbildung 2: Zytoarchitektonische Karte der lateralen und orbitalen Oberfläche des Menschen $(A)$ und des Makake - Affens (B) (Petrides und Pandya 2002, S.294)

Weiterhin wurde vorgeschlagen, der orbitofrontale Kortex solle aufgrund zytoarchitektonischer und funktioneller Beschaffenheiten als ein Teil des „orbitalen und medialen präfrontalen Kortex" angesehen werden (Ongur und Price 2000). 
Dieses Netzwerk besteht sowohl aus dem orbitofrontalen Kortex als auch aus Teilen des Gyrus cinguli.

Eine wichtige anatomische Besonderheit des orbitofrontalen Kortex stellen die interindividuellen Unterschiede im makroskopischen Aufbau dar. Beim Menschen wurden bisher mindestens drei sulkogyrale Muster identifiziert, wobei sich innerhalb dieser drei Typen noch weitere Subtypen differenzieren lassen. Grundsätzlich kann man von dem Vorhandensein von vier Sulci ausgehen: dem olfaktorischen, medialen, lateralen und transversalen orbitalen Sulcus (Chiavaras und Petrides 2000). Das Muster der Variabilitäten der orbitalen Sulci korreliert mit dem Zeitpunkt der embryonalen Entwicklung. So zeigen die Sulci, die sich zu einem späten Gestationszeitpunkt entwickeln, ein höheres Maß an interindividuellen Variabilitäten als solche, die sich früher entwickeln. Daraus lässt sich ein kaudorostraler Trend sowie ein mediolateraler Trend in der Sulkusvariabilität nachweisen, nach welchen die Variabilität der Muster von medial nach lateral sowie von kaudal nach rostral zunimmt (Chiavaras et al. 2001). Der Sulcus olfactorius unterliegt hierbei der geringsten Variabilität (Chiavaras und Petrides 2000).

Neben diesen Unterschieden im makroskopischen Aufbau, lassen sich ebenfalls welche in der Mikroskopie finden. Der histologische Aufbau stellt sich insofern als inhomogen dar, als dass die rostralen Anteile im Gegensatz zu den kaudalen Anteilen über eine granuläre Lamina IV verfügen. Diese granuläre Lamina im präfrontalen Kortex weisen nur Hominiden auf, die größte Ausprägung findet sich beim Menschen (Förstl 2005; Ongur und Price 2000).

Der orbitofrontale Kortex gehört jenem Teil des menschlichen Gehirns an, welches sich in der Ontogenese als letztes entwickelt. Zum Zeitpunkt der Geburt ist der präfrontale Kortex noch unreif, es lassen sich im Vergleich zum erwachsenen Gehirn eine höhere Zelldichte sowie dünnere kortikale Schichten nachweisen. Das Präfrontalhirn gehört zu den Gebieten, in denen der Prozess der Myelinisierung als letztes einsetzt, so finden sich hier beim Neugeborenen noch gar keine Myelinscheiden (Fuster 1980). 


\subsubsection{Projektionen}

Der orbitofrontale Kortex hat ausgeprägte reziproke Verbindungen mit sensorischen, limbischen, präfrontalen und motorischen Arealen (Cavada et al. 2000).

Unter den sensorischen Afferenzen lassen sich auditorische (Frey et al. 2004), gustatorische (Small et al. 1999), olfaktorische (Carmichael et al. 1994), somatosensorische (Rolls et al. 2003) und visuelle (Carmichael und Price 1995a) unterscheiden. Die zu einer Modalität gehörigen Afferenzen erreichen dabei jeweils separierte Areale des Kortexbereiches (Carmichael und Price 1996).

Interessant ist hierbei vor allem der Weg der gustatorischen Afferenzen. Diese nehmen ihren Ursprung von primären gustatorischen Arealen wie dem orbitofrontalen Operculum oder bestimmten Inselkortexarealen. Diese wiederum erhalten ihrerseits viszerale Afferenzen der Pars parvocellularis des Nucleus ventralis posteromedialis des Thalamus (Pritchard et al. 1986). Zusammen mit den gustatorischen Projektionen können also auch viszerale Afferenzen über diese Bahnen den orbitofrontalen Kortex erreichen (Carmichael und Price 1995b).

Es lassen sich verschiedenste Verbindungen mit Komponenten des limbischen Systems finden. Diese beinhalten unter anderem den Inselkortex, den Gyrus cinguli (Pandya et al. 1981), die entorhinalen und perirhinalen Kortizes (Carmichael und Price 1995b) sowie den Gyrus parahippocampalis (Barbas und Blatt 1995; Carmichael und Price 1995b; Morecraft et al. 1992). Die prominenteste aller Verbindungen zum limbischen System stellt allerdings ein Geflecht aus Projektionen zum Nucleus basalis der Amygdalakerngruppe dar (Carmichael und Price 1995b, 1996). Diese Projektionen sind topografisch organisiert und beinahe ausschließlich reziprok (Cavada et al. 2000).

Der orbitofrontale Kortex tauscht über drei verschiedene Wege Informationen mit dem Hippokampus aus. Erstens über die Verbindung mit dem entorhinalen Kortex (Carmichael und Price 1995b; Morecraft et al. 1992), zweitens über die Verbindung mit dem perirhinalen Kortex und dem Gyrus parahippocampalis (Carmichael und Price 1995b; Suzuki und Amaral 1994) und drittens besteht eine direkte, ipsilaterale Verbindung zwischen Hippokampus, Prosubikulum und 
orbitofrontalem Kortex. Hauptziel der hippokampalen Afferenzen stellt dabei der mediale Anteil des orbitofrontalen Kortex dar (Cavada et al. 2000).

Weiterhin lässt sich ein komplexes Verbindungsmuster mit den Thalamuskernen darstellen. Einige thalamische Kerne projizieren in den orbitofrontalen Kortex, die am besten untersuchte Verbindung ist hierbei die ipsilaterale Projektion der Pars magnocellularis des Nucleus mediodorsalis, die den orbitofrontalen Kortex von anderen präfrontalen Arealen abgrenzt (Cavada et al. 2000; Ray und Price 1993).

Der orbitofrontale Kortex unterliegt einer spezifischen Modulation von dopaminergen, noradrenergen und adrenergen Einflüssen der Thalamuskerne (Rico und Cavada 1998).

Die Verbindungen des orbitofrontalen Kortex mit motorischen Arealen sind weniger intensiv. Es finden sich Projektionen zu dem motorischen Teil des Gyrus cinguli (Carmichael und Price 1996) sowie mit den Kortexarealen, die die Augenmuskulatur repräsentieren (Mitz und Godschalk 1989). Weiterhin lassen sich Verbindungen zu den Basalganglien Claustrum, Striatum (Morecraft et al. 1992) und Substantia nigra (Ongur und Price 2000) finden.

Ferner gibt es Nervenbahnen zwischen dem orbitofrontalen Kortex und Hypothalamus (Ongur et al. 1998; Rempel-Clower und Barbas 1998) sowie dem Hirnstamm (Ongur und Price 2000). Auch andere präfrontale Kortexareale weisen reziproke Verbindungen mit dem orbitofrontalen Kortex auf (Barbas und Pandya 1989; Carmichael und Price 1995a).

Der orbitofrontale Kortex ist, so wie andere kortikale Regionen auch, innerviert von cholinergen und aminergen subkortikalen Fasern (Morecraft et al. 1992). Diese cholinergen Fasen stammen größtenteils aus dem Nucleus basalis Meynert. Der orbitofrontale Kortex zählt dabei, gemeinsam mit einigen wenigen Komponenten des limbischen Systems (Mesulam und Mufson 1984), zu den wenigen Strukturen, die ihrerseits selbst efferent in den Nucleus basalis Meynert projizieren und dadurch die Möglichkeit besitzen, den cholinergen Input in den gesamten cerebralen Kortex zu modifizieren (Mesulam und Mufson 1984; Ongur et al. 1998). 


\subsubsection{Aufgaben und Funktionen}

Der orbitofrontale Kortex gilt als Knotenpunkt der sensorischen Integration und Modulation autonomer Reaktionen. Er stellt einen wichtigen assoziativen Part des Lernprozesses und der Entscheidungsfindung dar (Kringelbach und Rolls 2004). Insbesondere findet die Bewertung von belohnenden und bestrafenden Stimuli im orbitofrontalen Kortex statt (Rolls 2004). Dieser Mechanismus kann als Grundvoraussetzung eines komplexen und emotionalen menschlichen Sozialverhaltens betrachtet werden und stellt damit einen Aspekt dar, der für die Entwicklung der menschlichen Spezies möglicherweise entscheidend war und ist (Kringelbach und Rolls 2004).

Der orbitofrontale Kortex beherbergt den sekundären und tertiären olfaktorischen Kortex (Carmichael et al. 1994; Rolls et al. 1996) sowie den sekundären gustatorischen Kortex (Rolls und Baylis 1994; Rolls et al. 1990). Der orbitofrontale Kortex kann als höheres gustatorisches Zentrum bezeichnet werden, in welchem multiple sensorische Inputs verknüpft werden und der einen wichtigen Teil bei der Generierung des Essverhaltens darstellt. (Small et al. 2007)

Als Schnittpunkt sensorischer Projektionen ist die Funktion des orbitofrontalen Kortex hochkomplex. Studien haben nicht nur gezeigt, dass die orbitofrontalen Neurone auf multisensorische Stimulation reagieren, sondern auch, dass das Ausmaß der Erregung in Relation zum affektiven Wert steht. Beispielsweise bietet eine Neuronengruppe eine stärkere Reizantwort auf Bild und Geruch eines bestimmten Nahrungsmittels, wenn der Proband hungrig darauf ist, als wenn er gesättigt ist (Critchley und Rolls 1996), dies wird auch als „sensorische Spezifität“ oder „selektive Sättigung“ bezeichnet (Kringelbach 2005).

Orbitofrontale gustatorische, olfaktorische und visuelle Neurone sind nur dann aktiv, wenn man hungrig ist. Also genau dann, wenn Geruch, Geschmack und Anblick von Nahrung erstrebenswert für den Organismus scheinen (Critchley und Rolls 1996). 
Der Weg der afferenten Projektionen in einer Hemisphäre des orbitofrontalen Kortex ist in Abbildung 3 anatomisch schematisch dargestellt. Die Fasern aus den peripheren sensorischen Arealen treten hauptsächlich im posterioren Teil des Kortex ein und werden dann auf ihrem Weg in die anterioren Teile des Areals weiterverarbeitet (Kringelbach 2005).

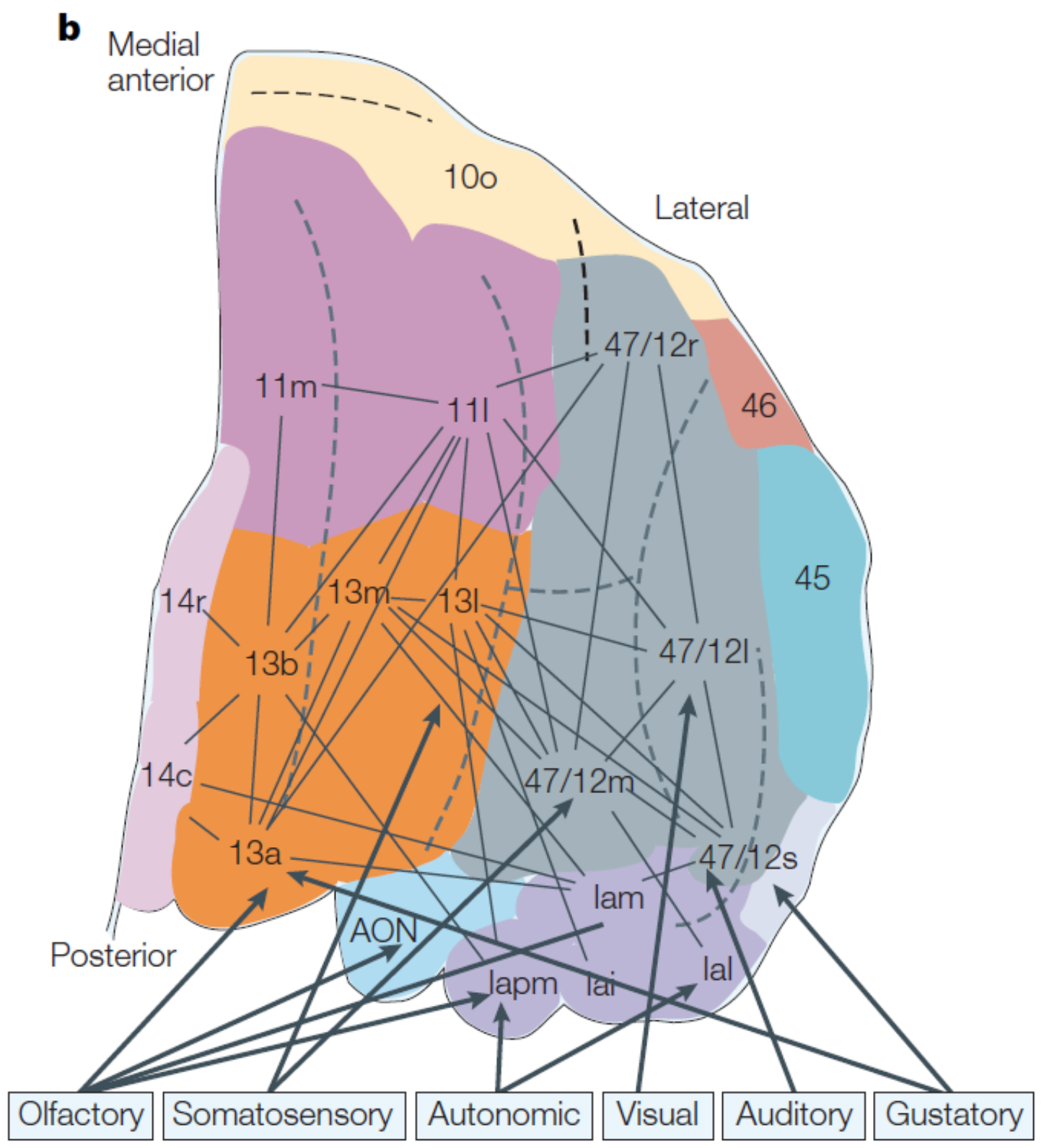

Abbildung 3: Schema der funktionellen Konnektivität des orbitofrontalen Kortex (Kringelbach 2005, S.700)

Das abstrahierte Modell des orbitofrontalen Kortex in Abbildung 4 verdeutlicht die Verarbeitung der Informationen innerhalb des Areals. Der Fluss der Informationen ist dabei aufsteigend dargestellt. Die sensorischen Afferenzen aus der Peripherie 
erreichen zuerst die primären sensorischen Areale des orbitofrontalen Kortex. Zunächst wird der Stimulus dekodiert und dann weitergegeben in die posterioren Teile des Kortex zur multimodalen Integration. Der belohnende Wert des Stimulus wird dann in den eher anterior liegenden Teilen bestimmt. Von hier aus kann diese Information an drei funktionell unterschiedliche Teile des anterioren Areals gelangen. Zum einen zum lateralen Anteil mit seinen Verbindungen zum anterioren Gyrus cinguli, wodurch die auf den Stimulus folgenden Handlungen des Individuums direkt beeinflusst werden. Der zweite Weg führt in die medialen Anteile des Areals, wo die Information als Erinnerung und Lernfortschritt gespeichert wird. Der dritte und letzte Weg führt in den mittleren Teil des anterioren Anteils, in welchem die Information zugänglich gemacht wird für bereits bestehende subjektive hedonische Erfahrungen.

Dabei ist zu beachten, dass die persönliche hedonische Erfahrung sowie der belohnenden Wert des Stimulus abhängig von Hunger, Durst oder anderen inneren Bedürfnissen ist (Kringelbach 2005).

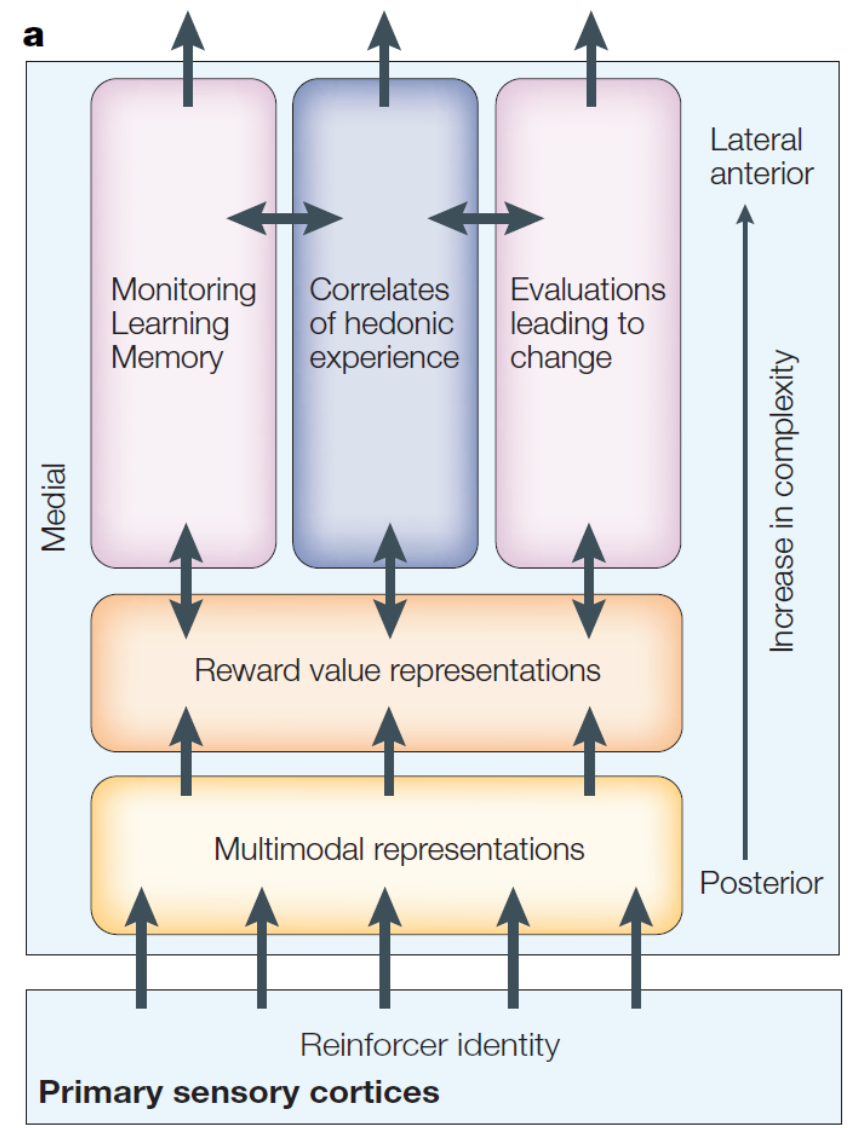

Abbildung 4: Funktionelles Modell des orbitofrontalen Kortex (Kringelbach 2005, S. 700) 
Besonders Area 11 der rechten Hemisphäre ist bedeutsam zur Einordnung und Wiedererkennung abstrakter visueller Informationen wie zum Beispiel das Erkennen und Wiedererkennen von menschlichen Gesichtern (Frey und Petrides 2003) sowie zur Geruchswahrnehmung (Zatorre et al. 1992). Die Lateralisierung auf die rechte Hemisphäre steht dabei im Einklang mit der allgemeinen Rolle der rechten Hemisphäre als Ort der Verarbeitung nonverbaler Informationen (Frey und Petrides 2000). Da die Lateralisierung erst auf dieser Ebene stattfindet, liegt es nahe, dass es sich hierbei um höhere assoziative Geschehnisse handelt. Bei der Verarbeitung von sensorischen Informationen im orbitofrontalen Kortex handelt es sich also nicht um einen initialen, sondern um einen sekundären oder gar tertiären Prozess (Zatorre et al. 1992).

Der orbitofrontale Kortex scheint ebenfalls eine entscheidende Bedeutung bei der Generierung von Affektverhalten zu spielen. Die genaue Rolle dabei ist weiterhin Gegenstand der aktuellen Forschung (Kringelbach und Rolls 2004).

\subsection{Der orbitofrontale Kortex im Kontext psychiatrischer Erkrankungen}

Funktionsminderungen des orbitofrontalen Kortex gehen mit sozialen und emotionalen Beeinträchtigungen einher (Hornak et al. 2003; Rolls et al. 1994). Insbesondere sind die Identifikation von non - verbalen Emotionen und Gesichtsmimik sowie das subjektive Emotionsempfinden gestört (Hornak et al. 2003). Darüber hinaus führen orbitofrontale Läsionen zu einer verminderten Reaktion auf belohnende oder bestrafende Konsequenzen einer Handlung (Elliott et al. 1997).

Läsionsstudien haben gezeigt, dass umschriebene Schäden am orbitofrontalen Kortex mit Defiziten in Emotion, Persönlichkeit, Verhalten und Sozialverhalten einhergehen. Die Patienten weisen ein mangelhaftes Affektverhalten sowie ein gestörtes Sozialverhalten auf und handeln mit mangelndem Verantwortungsbewusstsein (Hornak et al. 2003; Rolls et al. 1994). Die Patienten zeigen Schwächen bei der Interpretation sozialer Signale und der 
Wiedererkennung von menschlichen Gesichtern und Stimmen (Hornak et al. 2003; Hornak et al. 1996).

Erleiden Patienten eine Schädigung des orbitofrontalen Kortex schon in einem sehr jungen Lebensalter, so zeigen sie lebenslange Verhaltensstörungen, die sich resistent gegenüber verhaltenstherapeutischen Einflüssen zeigen. Ihnen scheint ein adäquates Wissen und Verständnis über moralische und gesellschaftlich akzeptierte Verhaltensweisen zu fehlen (Anderson et al. 1999). Patienten, die die Schädigung erst im höheren Lebensalter erleiden, behalten ihre Kenntnisse über moralische und gesellschaftliche Norm zwar bei, handeln aber nicht in jedem Fall entsprechend (Anderson et al. 1999). Dies impliziert, dass der orbitofrontale Kortex entscheidend ist bei der Entwicklung persönlicher moralischer Prinzipien. Weitere Läsionsstudien haben gezeigt, dass die Patienten mit einer orbitofrontalen Störung zur Perseveration neigen. Sie bemerken zwar eine veränderte Situation, können aber nicht angemessen darauf reagieren (Rolls et al. 1994).

Dysfunktionen oder volumetrische Alterationen des orbitofrontalen Kortex stehen im Zusammenhang mit dem Auftreten bestimmter neuropsychiatrischer Krankheiten wie Schizophrenie (Buchanan et al. 1998; llonen et al. 2000), Zwangsstörung (Carlsson 2001), Depression (Kalayam und Alexopoulos 1999; Nolan et al. 2002), Substanzmissbrauch (Liu et al. 1998), Störung des Sozialverhaltens (Raine et al. 2000), Autismus (Bachevalier und Loveland 2006; Dawson et al. 1998) sowie dem Tourette Syndrom (Peterson et al. 2001).

Einige Studien haben gezeigt, dass ungehemmtes und/oder apathisches Verhalten möglicherweise auf Störungen des orbitofrontalen Kortex zurückzuführen sind (Ongur und Price 2000).

\subsubsection{Schizophrenie}

Mitte des 20. Jahrhunderts rückte durch den Befund der Hypofrontalität (Ingvar und Franzen 1974) das Frontalhirn in den Mittelpunkt des Interesses der hirnbiologischen Schizophrenieforschung. Heute geht man von einem dysfunktionalen neuronalen Netzwerk bei der Schizophrenie aus, welches insbesondere den präfrontalen Kortex, den Inselbereich, den Fasciculus 
uncinatus, welcher Frontal- und Temporalhirn miteinander verbindet, und den Thalamus umfasst (Bagary et al. 2002; Bagary et al. 2003). Die Pathologie des orbitofrontalen Kortex stellt also nur einen Teilaspekt der Pathophysiologie der Schizophrenie dar (Förstl 2005).

Eine Studie zur Zell- und Kortexdichte im präfrontalen Kortex zeigte eine Gewebeschrumpfung bei erhaltener Zellzahl, was auf einen Verlust an Neuropil, bestehend aus Nervenfasern, Synapsen, Axonen und Dendriten, hinweist. Dies ist nicht als Folge einer neuroleptischen Behandlung zu interpretieren, denn diese kann genau gegenteilige Auswirkungen zeigen (Selemon und Goldman-Rakic 1999). Folglich handelt sich bei der Schizophrenie wahrscheinlich weniger um eine klassische neurodegenerative Erkrankung mit tatsächlichem Zelluntergang, sondern hauptsächlich um subtile Alterationen im subzellulären Bereich (Förstl 2005).

Ob diese Veränderungen auch mit volumetrisch messbaren Strukturveränderungen des orbitofrontalen Kortex einhergehen, ist bislang nicht eindeutig geklärt (Nakamura et al. 2008). So berichten einige Autoren über ein kleineres (Convit et al. 2001; Gur et al. 2000), andere über ein größeres Volumen (Lacerda et al. 2007) des orbitofrontalen Kortex bei Schizophrenen verglichen mit gesunden Kontrollpersonen. Kleinere Volumina des orbitofrontalen Kortex korrelierten dabei laut einigen Autoren mit dem Auftreten und der Schwere der Negativsymptomatik (Baare et al. 1999; Gur et al. 2000) und der sozialen Dysfunktion (Chemerinski et al. 2002; Gur et al. 2000). Im Gegenzug dazu korrelierte in einer anderen Studie von Hoptman und Mitarbeitern ein vergrößertes orbitofrontales Volumen mit einer gesteigerten Aggressivität (Hoptman et al. 2005).

In der Literatur ist speziell eine Verkleinerung der Area 11 des linken Kortex bei schizophrenen Probanden im Vergleich zu gesunden Kontrollpersonen gefunden worden, dabei korrelierte das Ausmaß der Verkleinerung in einer Studie mit der Krankheitsdauer und dem Ausmaß der formalen Denkstörung (Nakamura et al. 2008).

Von einigen Autoren wird diskutiert, ob der orbitofrontale Kortex als der einzige Teil des Frontalhirns anzusehen sei, in dem überhaupt eine Volumenreduktion im 
Sinne atrophischer Veränderungen bei Schizophrenie messbar sei (Lopez-Larson et al. 2002)

\subsubsection{Bipolare Störung}

Die Lebenszeitprävalenz für bipolare Störungen beträgt ca. $2 \%$, wobei die Patienten klassischerweise den Großteil der Erkrankung in einer depressiven Phase verbringen und weniger Zeit in der Manie (Kessler et al. 1997).

Das pathophysiologische Korrelat der bipolaren Erkrankung ist bis heute noch nicht vollständig verstanden. Es wird angenommen, dass im Vordergrund eine Störung im Netzwerk zwischen dem Präfrontalhirn und den Amygdalakernen sowie in einer Fronto- striato- thalamischen Verbindung steht (Altshuler et al. 2008). Der orbitofrontale Kortex scheint dabei jedoch eine Schlüsselrolle einzunehmen (Nery et al. 2009). Postmortale Studien haben verschiedene neuropathologische Veränderungen in diesem Kortexareal zeigen können, beispielsweise eine reduzierte Neuronenzahl (Cotter et al. 2005)) sowie eine Reduktion des sauren Gliafaserproteins GFAP (glial fibrillary acidic protein), was auf eine Dysfunktion der Astrozyten hinweisen könnte (Toro et al. 2006). Studien mit funktionellen kraniellen Bildgebungen haben sowohl eine gesteigerte Aktivität im orbitofrontalen Kortex (Elliott et al. 2004) als auch in den Amygdalakernen (Altshuler et al. 2005) gezeigt.

Insgesamt deutet demzufolge vieles darauf hin, dass bei bipolaren Patienten eine Funktionsstörung des orbitofrontalen Kortex vorliegt. Trotzdem wurde bei den Studien, die sich bisher mit den rein volumetrischen Veränderungen des orbitofrontalen Kortex bei bipolaren Patienten beschäftigt haben, keine einheitlichen Ergebnisse erzielt. In einer aktuellen Studie von (Nery et al. 2009) wurde der orbitofrontale Kortex von zum Zeitpunkt der Bildgebung euthymen, manischen und depressiven Patienten manuell vermessen. Dabei konnte bei den depressiven Patienten ein signifikant verkleinerter orbitofrontaler Kortex gefunden werden. 


\subsubsection{Zwangsstörung}

Die Zwangstörung ist eine chronische Erkrankung mit einer Prävalenz von 2-3\% (Karno et al. 1988). Sie zeichnet sich aus durch sich aufdrängende Gedanken, die mit Angst verbunden sind. Die auf diese Zwangsgedanken folgenden Zwangshandlungen haben zum Ziel, diese Angst zu lösen oder gar zu neutralisieren. Die Angstlinderung ist jedoch nicht dauerhaft und führt im Sinne einer operanten Konditionierung zu zyklischen Wiederholungen der Zwangshandlung (Rotge et al. 2008).

Es gibt verschiedene Modelle die sich mit der der Zwangserkrankung zugrunde liegenden Pathophysiologie auseinandersetzen. Alle stimmen darin überein, dass es zu einer Dysfunktion der Verbindung zwischen den limbischen Arealen des Präfrontalkortex und den Basalganglien kommt. Diese Projektionen führen durch mediale Anteile des Thalamus. In den verschiedenen Modellen variiert jedoch jeweils die genaue Lokalisation der Störung (Aouizerate et al. 2004).

Als funktionelles Korrelat lässt sich im Vergleich mit gesunden Kontrollpersonen unter anderem eine gesteigerte Aktivität im orbitofrontalen Kortex feststellen (Kwon et al. 2003). Nach einer erfolgreichen Therapie der Erkrankung zeigt sich die Aktivität hingegen vermindert (Saxena et al. 1999). Das legt nahe, dass der orbitofrontale Kortex zum Entstehen der Symptomatik beiträgt (Choi et al. 2004).

Roth et al. (2007) konnten in einer funktionellen MRT- Studie während einer Impulsunterdrückung bei Zwangspatienten eine negative Korrelation zwischen der Aktivität des orbitofrontalen Kortex und dem Schwergrad der Symptomatik zeigen. Hingegen konnte eine positive Korrelation zwischen der thalamischen Aktivität und dem Schweregrad der Symptomatik gezeigt werden. Bei gesunden Kontrollpersonen konnte dies nicht dargestellt werden Dieses abweichende Muster legt nahe, dass funktionelle Anomalien des orbitofrontalen Kortex und des Thalamus eng, aber spiegelbildlich mit der Ausprägung einer Zwangsstörung verbunden sind.

Neben pathologisch funktionellen Befunden konnten bereits auch volumetrische Alterationen des orbitofrontalen Kortex im strukturellen Kernspintomogramm bei Zwangskranken gezeigt werden (Kim et al. 2001). Zwischen den verschiedenen 
Studien, die sich mit den volumetrischen Alterationen beschäftigt haben, konnten jedoch nur heterogene Ergebnisse erzielt werden. Insbesondere trifft dies für Volumenveränderungen des Thalamus zu. In einer aktuellen Metaanalyse konnten Rotge et al. (2009) indes darlegen, dass in der Mehrzahl der Studien die Zwangspatienten im Vergleich mit Kontrollpersonen kleinere orbitofrontale sowie größere thalamische Volumina gezeigt haben.

\subsection{Studienziel}

Der orbitofrontale Kortex spielt bei den im vorangegangenen Abschnitt genannten Erkrankungen eine Rolle in der Pathophysiologie. Volumetrische Auffälligkeiten eben dieses Kortexareals sind neben der Schizophrenie auch für die bipolare Störung und für die Zwangserkrankung beschrieben worden.

Allerdings existieren noch keine Diagnose - übergreifenden volumetrischen Vergleichsstudien, um den Stellenwert dieser Veränderungen besser einordnen und eine eventuelle Diagnosespezifität nachweisen zu können.

In der vorliegenden Arbeit soll das Volumen des orbitofrontalen Kortex an Stichproben von schizophren ersterkrankten Patienten, euthymen bipolaren Patienten, Zwangspatienten und gesunden Kontrollpersonen verglichen werden.

Damit soll in der vorliegenden Dissertation insbesondere untersucht werden,

o ob ein volumetrischer, ein- oder beidseitiger Unterschied des orbitofrontalen Kortex zwischen ersterkrankten schizophrenen Patienten, bipolaren Patienten, Zwangspatienten und gesunden Kontrollpersonen besteht.

Weiterhin soll ermittelt werden,

o ob eine Asymmetrie des orbitofrontalen Kortex z. B. zugunsten der rechten Hemisphäre bei den Patientengruppen vermindert oder aufgehoben ist.

Ferner sollen die

- Zusammenhänge und der Einfluss intervenierender Variablen wie Alter, Geschlecht und Bildung auf die Hirnstruktur des orbitofrontalen Kortex dargestellt werden. 
Darüber hinaus soll gezeigt werden,

o ob es volumetrische Unterschiede im orbitofrontalen Kortex bei schizophrenen Patienten mit positiver Familienanamnese für Schizophrenie gibt im Gegensatz zu Patienten ohne familiäre Belastung.

Bisher wurden in der Literatur überwiegend Volumenveränderungen des orbitofrontalen Kortex bei chronisch schizophrenen Patienten beschrieben.

In der vorliegenden Dissertation soll deswegen insbesondere darauf geachtet werden,

o ob Veränderungen auch schon bei an Schizophrenie Ersterkrankten nachweisbar sind und

o ob eine „Krankheitsspezifität" vorliegt oder sich ähnliche Volumenreduktionen auch bei anderen Krankheitsbildern wie der bipolaren Erkrankung oder der Zwangsstörung finden lassen, daher der Diagnose - übergreifende Ansatz. 


\section{Material und Methoden}

\section{$2.1 \quad$ Patientenkollektiv}

Untersucht wurden die MRT- Schnittbilder von 36 Patienten mit der Erstmanifestation einer Schizophrenie nach ICD-10 (F20.09), 39 Patienten mit einer bipolaren Störung nach ICD-10, derzeit euthym (F31.7), und 27 Patienten mit einer Zwangsstörung nach ICD-10 (F42) im Vergleich zu einer gesunden Kontrollgruppe von 37 Personen.

Die MRT- Aufnahmen stammen alle von Probanden aus dem Patientenkollektiv der Klinik für Psychiatrie und Psychotherapie des Universitätsklinikums des Saarlandes. Die Probanden bzw. Patienten wurden vor Beginn der Studie ausführlich aufgeklärt und gaben schriftlich Ihre Einwilligung zur Teilnahme (Informed Consent). Für die Studie lag ein positives Votum der zuständigen EthikKommision, Ärztekammer des Saarlandes, vor.

Das Vorhandensein einer Minderbegabung, einer Demenz oder einer organischen ZNS- Erkrankung galt als Ausschlusskriterium für die Aufnahme in die Studie. Alle Patienten wurden zum Zeitpunkt der MRT- Untersuchung mit verschiedenen Psychopharmaka behandelt. Dazu zählten bei den schizophrenen Patienten Neuroleptika als antipsychotisch medikamentöse Therapie und bei den Probanden der anderen Diagnosegruppen Antidepressiva, Mood Stabilizer oder ebenfalls Antipsychotika.

Beim Gesamtkollektiv von 139 Probanden wurde Alter, Geschlecht, Ausbildung und Händigkeit (Oldfield 1971) erhoben.

Bei den Bipolaren und den Zwangserkrankten wurde zusätzlich auch das Alter bei Auftreten sowie die Dauer der Krankheit protokolliert.

Darüber hinaus wurde bei den schizophren Erkrankten das Vorliegen von erkrankten Familienmitgliedern ersten und zweiten Grades, die Dauer des unbehandelten Prodroms bzw. der Psychose bis zur ersten Untersuchung, der PANSS Score (Positive and Negative Syndrome Scale), der CGI (Clinical Global 
Impressions Score), die GAF-Skala (Global Assessment of Functioning) und die MMSE (Mini Mental State Examination) erhoben. Alle schizophrenen Patienten wurden mit atypischen Neuroleptika, sogenannten Antipsychotika der zweiten Generation, behandelt. Die Tagesdosis als auch die kumulative Gesamtdosis bis zum MRT- Untersuchungstag wurden in Chlopromazin - Äquivalente (CPZ) (Woods 2003) umgerechnet und in die Auswertung einbezogen.

Bei den bipolaren Patienten wurde die Anzahl der depressiven und manischen Episoden, der MADRS und YMRS, die Einnahme von Lithium und Neuroleptika sowie das Vorhandensein einer Psychose in die Auswertung einbezogen.

Tabelle 1: Soziodemografische und klinische Daten des Gesamtkollektivs

\begin{tabular}{|c|c|c|c|c|}
\hline & $\begin{array}{c}\text { Kontrollen } \\
(N=37)^{a}\end{array}$ & $\begin{array}{l}\text { Schizophrene } \\
\qquad(N=36)^{a}\end{array}$ & $\begin{array}{l}\text { Bipolare } \\
(N=39)^{a}\end{array}$ & $\begin{array}{c}O C D \\
(N=27)^{a}\end{array}$ \\
\hline Alter & $34,2 \pm 10,3$ & $29,3 \pm 7,5$ & $43 \pm 11,9$ & $35,1 \pm 9,8$ \\
\hline Geschlecht (M:F) & $15: 22$ & $24: 12$ & 19:20 & $13: 14$ \\
\hline Ausbildung $^{I}$ & $15 \pm 2,7$ & $12,9 \pm 2,8$ & $13,9 \pm 2,9$ & $13,3 \pm 2,4$ \\
\hline Händigkeit (re:li) & $31: 7$ & $30: 6$ & $34: 5$ & $24: 3$ \\
\hline $\begin{array}{l}\text { Schulabschluss } \\
\text { (HS:RS:AB:ST) }\end{array}$ & 4:10:10:13 & $10: 7: 7: 3$ & $12: 7: 10: 10$ & $7: 12: 3: 5$ \\
\hline $\begin{array}{c}\text { Alter bei Auftreten } \\
\text { der Krankheit }^{1}\end{array}$ & & & $27,94 \pm 8,23$ & $21,56 \pm 10,87$ \\
\hline Krankheitsdauer $^{I}$ & & & $14,32 \pm 10,43$ & $13,67 \pm 9,58$ \\
\hline $\begin{array}{c}\text { Anzahl depressiver } \\
\text { Episoden }\end{array}$ & & & $7,54 \pm 7,41$ & \\
\hline $\begin{array}{c}\text { Anzahl manischer } \\
\text { Episoden }\end{array}$ & & & $8,11 \pm 8,32$ & \\
\hline MADRS & & & $4,66 \pm 3,47$ & \\
\hline YMRS & & & $2,59 \pm 2,85$ & \\
\hline Lithium (nein:ja) & & & $22: 16$ & \\
\hline Psychose (nein:ja) & & & $27: 11$ & \\
\hline $\begin{array}{c}\text { Neuroleptika } \\
\text { (n:k:a) }\end{array}$ & & & $19: 4: 14$ & \\
\hline $\begin{array}{c}\text { Schizophrenie in } \\
\text { FA (nein:ja) }\end{array}$ & & $26: 10$ & & \\
\hline Dauer der & & $45,72 \pm 59,32$ & & \\
\hline
\end{tabular}




\begin{tabular}{|c|c|c|c|c|}
\hline & $\begin{array}{c}\text { Kontrollen } \\
(\mathrm{N}=37)^{a}\end{array}$ & $\begin{array}{c}\text { Schizophrene } \\
(\mathrm{N}=36)^{a}\end{array}$ & $\begin{array}{c}\text { Bipolare } \\
(\mathrm{N}=39)^{a}\end{array}$ & $\begin{array}{c}\text { OCD } \\
(\mathrm{N}=27)^{a}\end{array}$ \\
\hline Psychose bis $\mathrm{U}^{2}$ & & & & \\
\hline Prodromi bis U1 & & $180,69 \pm 165,59$ & & \\
\hline PANSS total & & $89,11 \pm 17,82$ & & \\
\hline CGI & & $5,63 \pm 0,69$ & & \\
\hline GAF & & $33,37 \pm 12,82$ & & \\
\hline MMSE & & $29,47 \pm 1,13$ & & \\
\hline CPZ Tagesdosis & & $340,6 \pm 356,2$ & & \\
\hline
\end{tabular}

Legende: ${ }^{\text {a: }}$ Mittelwert und Standardabweichung, sofern nicht anders angegeben; HS: Hauptschule; RS: Realschule; AB: Abitur; ST: Studium; FA: Familienanamnese; n:k:a: nein:konventionell:atypisch; ${ }^{1}$ : In Jahren; 2 : in Wochen.

\subsection{Beschreibung der psychopathologischen Skalen}

Eine grobe Abschätzung der kognitiven- mnestischen Funktionen wurde mit der MMSE (Mini Mental State Examination) (Folstein et al. 1975) vorgenommen. Die Erhebung der MMSE Score diente unter anderem auch dazu, Zeichen dementiver Erkrankungen zu erkennen um solche Patienten dann aus der Studie auszuschließen zu können.

Die PANSS gehört seit ihrer Entwicklung von Kay et al. (1987) zu den maßgebenden Skalen zur Erfassung der Psychopathologie in der Schizophrenieforschung. Die Skala beurteilt die positiven und negativen Dimensionen der schizophrenen Erkrankung. Unter Positivsymptomatik werden Wahn, Halluzination, beispielsweise akustische, optische und den eigenen Körper betreffende Halluzinationen, und Ich-Erlebnisstörungen wie Gedankeneingebung, -ausbreitung und -entzug gezählt. Zur Negativsymptomatik zählt man Antriebsmangel, Affektstörungen, Alogie, Anhedonie, Mangel an sozialen Kontakten sowie Konzentrationsstörungen. Diese Symptome werden in einem 30bis 40-minütigen formalisierten psychiatrischen Interview anhand einer siebenstufigen Skala von 1 (nicht vorhanden) bis 7 (extrem ausgeprägt) bewertet. Jedes Symptom ist einer von drei Skalen zugeordnet, sieben der Positivskala, weitere sieben der Negativskala und die restlichen 16 der Globalskala. Diese Beurteilung bezieht sich auf die Befindlichkeit der Person während der vorangegangenen sieben Tage. Es können ebenfalls Informationen durch das 
Krankenhauspersonal oder Angehörige mit in die Bewertung einfließen. Aus der PANSS ergibt sich ein Summenscore von 30 bis 120 Punkten.

Die GAF - Skala dient der globalen Beurteilung des Funktionsniveaus in sozialen und beruflichen Bereichen. Dabei können Werte von 0 bis 100 Punkte erzielt werden. Die Abstufung der Skala erfolgt jeweils in 10er Bereichen. Von einer nahezu unbeeinträchtigten Leistungsfähigkeit ist beispielsweise bei einem Wert ab 80 Punkten auszugehen. Diese Skala wurde dem Diagnostischen und Statistischen Manual IV (Saß et al. 1996) entnommen.

Die CGI - Skala ist eine Möglichkeit, den klinischen Zustand bzw. den Schweregrad der Erkrankung des Patienten mit Hilfe von 8 Stufen zu beurteilen. Dabei reicht die Skala von 0 (nicht zu beurteilen), über 1 (überhaupt nicht krank) bis hin zu 7 (extrem schwer krank) (CIPS 1986).

Bei den bipolaren Patienten wurden die Anzahl der depressiven und manischen Episoden protokolliert. Außerdem wurde jeweils der Score in der Montgomery Asberg Depression Rating Scale (MADRS) und in der Young Mania Rating Scale (YMRS) Score erhoben.

Der MADRS gibt einen Schweregrad für die Depression an und ist unterteilt in zehn Bereiche wie Traurigkeit, Innere Anspannung, Appetit oder Konzentrationsschwierigkeiten. In jedem Teilbereich gibt es eine Punktzahl von 0 (keine Probleme) bis 6 (sehr starke Probleme), woraus sich ein Summenscore von maximal 60 Punkten ergibt (Montgomery und Asberg 1979).

Die YMRS gibt einen Score an für den Schweregrad der Manie. Die YMRS besteht aus 11 Fragen, welche sich auf die vorangegangenen 48 Stunden beziehen. In den Fragen werden unter anderem manische Symptome wie vermindertes Schlafbedürfnis, gesteigerte Energie, gehobene Stimmung und Antrieb, vermehrte Reizbarkeit, aggressives Verhalten und veränderte Denkinhalte abgefragt. Je stärker ein Symptom ausgeprägt ist, desto mehr Punkte werden pro Frage verliehen. Bei den Fragen 6, 8 und 9 können bis zu 8 Punkte vergeben werden, bei allen anderen Fragen bis zu 4 Punkte mit Abstufungen von einem halben Punkt. Nicht nur die Angaben des Patienten, sondern auch der klinische 
Eindruck und Angaben von Angehörigen können in die Bewertung einfließen. Insgesamt können 60 Punkte erreicht werden (Young et al. 1978).

\subsection{MRT- Acquisition}

Die MRT- Untersuchungen wurden an einem 1,5 Tesla Scanner der Firma. Siemens, Typ Sonata, in der Abteilung für Neuroradiologie des Universitätsklinikums des Saarlandes durchgeführt. Die volumetrischen Auswertungen erfolgten an T1- gewichteten Bildern, Sequenz IR/GR als MPRAGE (Magnetization Prepared Rapid Gradient Echo), Typ 3D, Voxel 1 × 1 × $1 \mathrm{cmm}$; Matrix 176 × 256 × 256, mit den Zeiten TR = 1900 ms; TE = 4 ms; TI = 700 ms; Flip-Winkel 15 Grad.

\subsection{Volumetrie}

Die Volumetrie erfolgte mit dem frei verfügbaren Programm MRICroN, einem Imaging Tool, welches ANALYZE-Formate, DICOM, NEMA, GE, Interfile, ECAT, Picker, Siemens, Elscint, VFF, VoxBo und Rohbilddaten verwerten kann. Mit MRIcroN kann auch in SPM konvertiert werden. Die Visualisierung schließt Standardschnitte, schiefe Schnittebenen und Volumenmarkierungen ein. Mit MRIcroN können bestimmte Regionen manuell markiert und die umfahrene Fläche gemessen werden. Bei mehreren Schnitten wird durch die Multiplikation der Schichtdicke von $1 \mathrm{~mm}$ mit der Gesamtzahl der markierten Voxel mit einer Fläche von jeweils $1 \mathrm{~mm}^{2}$ in den einzelnen Schichten das Volumen (in $\mathrm{mm}^{3}$ oder $\mathrm{cmm}$ ) ermittelt. Dies wird auch als Region of Interest (ROI) bezeichnet.

Das Programm wurde von Chris Rorden; University of South Carolina, entwickelt. Internet Adresse: http://www.sph.sc.edu/comd/rorden/mricron/.

Das Gesamtvolumen der grauen Substanz wurde in einem semiautomatisierten Messverfahren erfasst, wobei die Software SPM99 (Statistical Parametric Mapping, Wellcome Department of Cognitive Neurology, London, UK) verwendet 
wurde. SPM ist eine Software zum Berechnen von fMRT- und PET-Daten, die als MATLAB- Application programmiert ist. Nach dem Preprocessing wurden mit Hilfe der Segment Funktion von SPM99 (http://www.fil.ion.ucl.ac.uk/spm/) die T1 gewichteten MRT- Scans graue Substanz, weiße Substanz und Liquorräume segmentiert. Anschließend konnte das Volumen der grauen Substanz anhand der segmentierten Voxelzahl mit MATLAB berechnet werden.

Das gesamte Volumen des präfrontalen Kortex (PFC) wurde mit einer automatisierten Parcellationsprozedur berechnet, welche in der Software zur Berechnung des automaisierten Gyrifizierungsindex der Arbeitsgruppe um Prof. Stephen Lawrie der Universität Edinburgh implementiert ist. Die Volumenberechnungen wurden mit den übersandten MRT- Scans im AnalyzeFormat direkt in Edinburgh von dem Entwickler des Verfahrens, Thomas William Moorhead, vorgenommen (Bonnici et al. 2007).

\subsection{Identifikation des orbitofrontalen Kortex}

Die Ausdehnung des orbitofrontalen Kortex wurde größtenteils in der Koronarebene bestimmt, siehe Abbildungen 5 und 6. Die Festlegung der Grenzen erfolgte in Anlehnung an eine Übersichtsarbeit und Studie von Crespo-Facorro et al. (1999). 


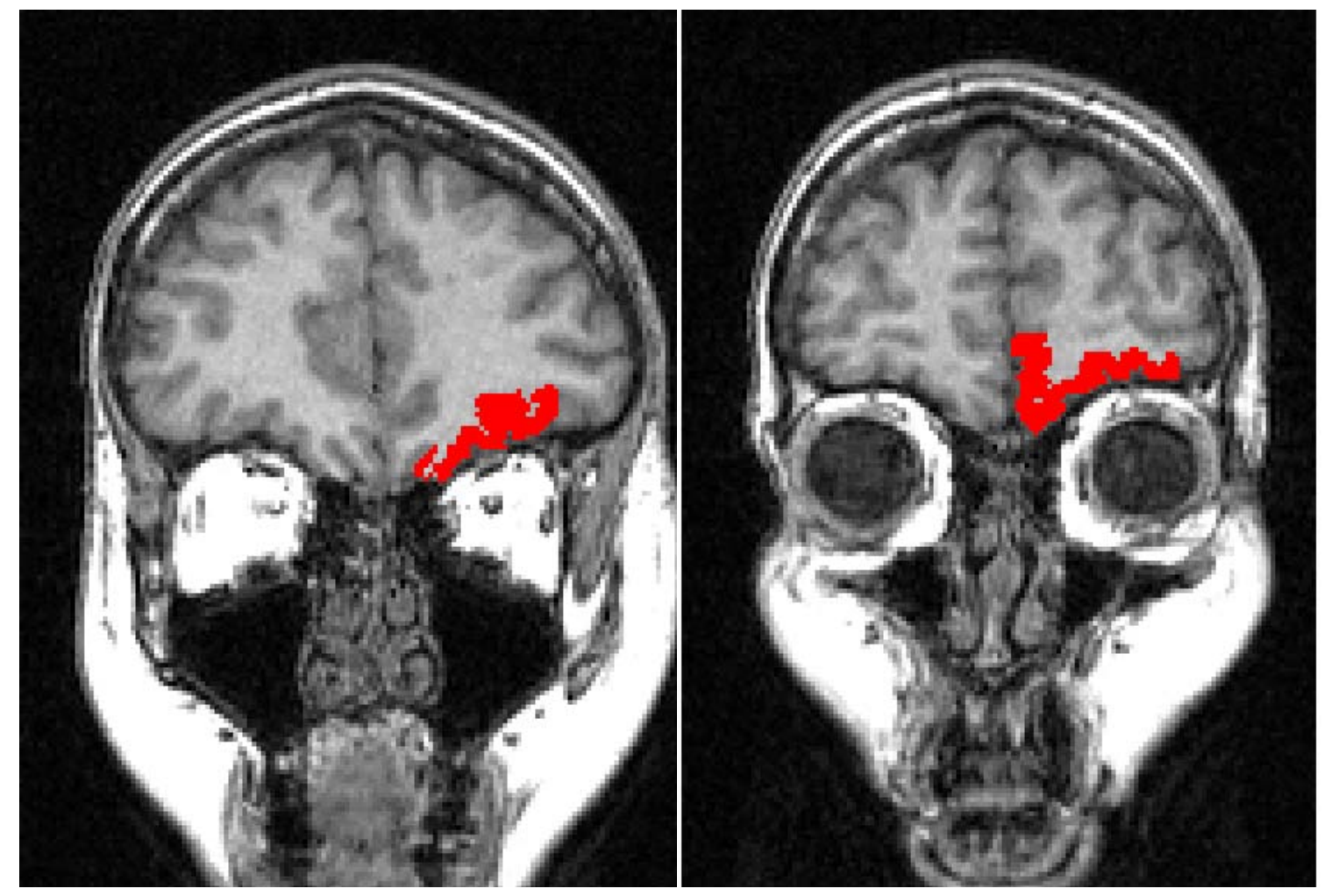

Abbildungen 5 und 6: Markierung der grauen Substanz OFC links

\subsubsection{Start- und Endschicht}

Die Markierung des Areals erfolgt von kaudal nach rostral. Um die Startschicht zu definieren, wird im Sagittalschnitt eine senkrechte Linie durch den am weitesten rostral gelegenen Punkt des äußeren Knies gezogen. Alle Koronarschnitte, die rostral dieser Markierung liegen, werden zum Areal gezählt.

Die Endschicht ist der letzte Schnitt, auf dem noch Teile des rostralen Frontalhirns zu sehen sind. Sobald jedoch auf den Koronarschnitten keine sinnvolle Markierung mehr möglich ist weil die Gyri am rostralen Pol nicht mehr voneinander abzugrenzen sind, wird die Markierung im Sagittalschnitt beendet. Hier werden die bereits markierten Gyri sinnvoll bzw. mit horizontalen Linien vervollständigt bis der Frontalpol endet.

\subsubsection{Laterale Grenze}

Als laterale Begrenzung wird der Sulcus orbitalis lateralis (LOS) definiert. Ist dieser auf den rostralen Schnitten nicht mehr zu finden, dient der frontomarginale Sulcus 
(FMS) als laterale Grenze. Ist der LOS auf den kaudalen Schnitten nicht mehr zu sehen, ist der tiefste Punkt des circular insular Sulcus (CSI) grenzbildend. Sind auf einem Schnitt weder CSI noch LOS zu sehen, dienen im Sagittalschnitt horizontal gezogene Linien durch die tiefsten Punkte des ersten erkennbaren LOS als laterale Begrenzung.

\subsubsection{Mediale Grenze}

Die mediale Begrenzung wird im kaudalen und mittleren Teil des Bereiches durch den Sulcus orbitalis gegeben. Sobald dieser in den rostralen Schnitten verschwindet, dient der tiefste Punkt des Sulcus rostralis superior (SRS) als Grenze.

\subsubsection{Weiße Substanz}

Die weiße Substanz wird ebenfalls in der Koronarebene bestimmt. Als Vorlage dient dabei die vorhergehend markierte graue Substanz. Von der lateralen und medialen Grenze werden jeweils gerade Linien gezogen. Die hierbei eingeschlossene Grenze wird als die weiße Substanz des orbitofrontalen Kortexes definiert.

Ist der Sulcus orbitalis medial grenzbildend, wird eine senkrechte Linie gezogen, ist es der FMS wird eine horizontale Linie gezeichnet. Von allen lateralen Grenzen werden horizontale Linien zur Grenzbildung gezogen.

Sollten von medial und lateral horizontale Linien gezogen werden, wird die höhere als grenzbildend angesehen.

\subsection{Statistik}

Für die statistische Auswertung wurde das Programm SPSS, Version 16.0 verwendet. Alle Auswertungen wurden mit dem Signifikanzniveau $\alpha=0,05$ durchgeführt. 
Zunächst erfolgten Subgruppenanalysen der drei Diagnosegruppen Schizophrenie, bipolare Störung und Zwangserkrankung in Bezug auf die gesunde Kontrollgruppe.

Als intervenierende Variablen wurden hierbei untersucht:

o Alter

o Geschlecht

o Ausbildungsdauer

o Händigkeit

- Art des Schulabschlusses (Hauptschule, Realschule, Abitur, Studium)

o Für die schizophren Ersterkrankten: Positive Familienanamnese für Schizophrenie, Krankheitsdauer von den ersten psychotischen Symptomen bis zur Diagnosestellung (Psychosedauer), von den ersten Vorläufersymptomen bis zur Behandlung (Prodromdauer), die Werte der Positive And Negative Syndrome Scale (PANSS), Clinical Global Impressions (CGI), Global Assessment of Functioning (GAF), Mini Mental State Examination (MMSE) sowie Chlorpromazin Tagesdosis (CPZ).

o Für die Bipolare Störung: Ersterkrankungsalter, Krankheitsdauer, Anzahl depressiver und manischer Episoden, Montgomery Asberg depression rating scale (MADRS), Young Mania Rating Scale (YMRS), das Vorhandensein von psychotischen Symptomen, Therapie mit Neuroleptika, Lithium und anderen Stimmungsaufhellern.

o Für die Zwangserkrankten: Ersterkrankungsalter und Krankheitsdauer.

Bei der anschließenden Diagnose - übergreifenden Auswertung wurde anhand der gefundenen relevanten Einflussfaktoren eine multivariante Kovarianzanalyse (MANCOVA) durchgeführt. Als abhängige Variablen wurden die Volumina des orbitofrontalen Kortex, teilweise bezogen auf Gesamt- oder Präfrontalkortex, und als unabhängige Variablen die Diagnosegruppen betrachtet.

Um Aussagen über das Ausmaß der relativen Größenveränderungen des orbitofrontalen Kortex machen zu können, wurden auch Analysen unter Bezugnahme des zerebralen Gesamtvolumens der grauen bzw. weißen Substanz 
sowie des präfrontalen Volumens gemacht. Berücksichtigt wurde hierbei wieder sowohl die graue als auch die weiße Substanz. 


\section{Ergebnisse}

\section{1 Überprüfung der Reliabilität}

Um ein Maß für die Güte des gewählten Testverfahrens zu finden, wurden bei einem Set von 10 MRT Scans zwei verschiedene Test zur Bestimmung der Reliabilität angewandt.

\subsubsection{Retest}

Bei zehn Gehirnen wurde durch den gleichen Tester ein blinder Retest durchgeführt und das Volumen des linken orbitofrontalen Kortex doppelt vermessen. Bei der anschließenden Berechnung des IntraklassenKorrelationskoeffizienten (ICC) ergaben sich zufriedenstellend hohe Werte $>0,9$ (siehe Tabelle 2).

Tabelle 2: Retest ICC

\begin{tabular}{|c|c|c|c|}
\hline & & Pearson-Korr. & ICC \\
\hline \multirow[t]{3}{*}{ Links grau } & $r$ & 0,994 & 0,992 \\
\hline & $p$ & $>0,0005$ & \\
\hline & $n$ & 10 & \\
\hline \multirow[t]{3}{*}{ Links weiss } & $r$ & 0,987 & 0,986 \\
\hline & $p$ & $<0,0005$ & \\
\hline & $n$ & 10 & \\
\hline \multirow[t]{3}{*}{ Links total } & $r$ & 0,996 & 0,993 \\
\hline & $p$ & $<0,0005$ & \\
\hline & $n$ & 10 & \\
\hline
\end{tabular}

Legende: Pearson-Korr.: Pearson Korrelationskoeffizient; ICC: Intraklassen- Korrelationskoeffizient.

\subsubsection{Interrater}

Ebenfalls bei zehn Gehirnen wurde ein Interrater Test durchgeführt. Die Gehirne wurden blind von einem zweiten Tester untersucht. Auch hier ist der ICC zufriedenstellend hoch mit Werten > 0,9 (siehe Tabelle 3). 
Tabelle 3: Interrater ICC

\begin{tabular}{|c|c|c|c|}
\hline & & Pearson-Korr & $I C C$ \\
\hline \multirow[t]{3}{*}{ Links grau } & $r$ & 0,994 & 0,986 \\
\hline & $p$ & $>0,0005$ & \\
\hline & $n$ & 10 & \\
\hline \multirow[t]{3}{*}{ Links weiss } & $r$ & 0,948 & 0,943 \\
\hline & $p$ & $<0,0005$ & \\
\hline & $n$ & 10 & \\
\hline \multirow[t]{3}{*}{ Links total } & $r$ & 0,984 & 0,975 \\
\hline & $p$ & $>0,0005$ & \\
\hline & $n$ & 10 & \\
\hline
\end{tabular}

Legende: Pearson-Korr.: Pearson Korrelationskoeffizient; ICC: Intraklassen- Korrelationskoeffizient.

\subsection{Vergleich der volumetrischen Größe des orbitofrontalen Kortex bei den verschiedenen Patientengruppen}

Da bei drei Probanden aus dem ursprünglichen Gesamtkollektiv die anatomischen Grenzen des orbitofrontalen Kortex nicht eindeutig bestimmt werden konnten, wurden diese in den Studienergebnissen nicht berücksichtigt.

\subsubsection{Intervenierende Variablen}

Als intervenierende Variablen wurden bei allen Probanden Geschlecht, Händigkeit, Alter und Bildungsdauer überprüft.

Es zeigten sich signifikante Volumenunterschiede in Abhängigkeit des Geschlechts. So war bei den weiblichen Patienten das Volumen der totalen zerebralen Substanz sowie des Präfrontalkortex, jeweils für graue und weiße Substanz, mit $p<0,0005$ signifikant kleiner als bei den männlichen. Ebenfalls der orbitofrontale Kortex zeigte sich bei den weiblichen Patienten signifikant kleiner als bei den männlichen (siehe Tabelle 4). 
Tabelle 4: Geschlechtsabhängige Volumina aller Krankheitsgruppen

\begin{tabular}{|c|c|c|c|c|c|c|c|}
\hline & $\begin{array}{c}\text { Kontrollen } \\
(\mathrm{m}=15 ; \mathrm{w}=22)^{\mathrm{a}}\end{array}$ & $\begin{array}{c}\text { Schizophrene } \\
(=21 ; \mathrm{w}=12)^{\mathrm{a}}\end{array}$ & $\begin{array}{c}\text { Bipolare } \\
(\mathrm{m}=11 ; \mathrm{w}=16)^{\mathrm{a}}\end{array}$ & $\begin{array}{c}\text { OCD } \\
(\mathrm{m}=12 ; \mathrm{w}=14)^{\mathrm{a}}\end{array}$ & $\mathrm{df}$ & $\mathrm{F}$ \\
\hline $\begin{array}{c}\text { Totale graue } \\
\text { Substanz }\end{array}$ & $-4,2 \%$ & $-8,3 \%$ & $-7,6 \%$ & 1,115 & 15,49 & $<0,0005$ \\
\hline $\begin{array}{c}\text { Totale weiße } \\
\text { Substanz }\end{array}$ & $-14,2 \%$ & $-8,1 \%$ & $-16,4 \%$ & $-10,9 \%$ & 1,115 & 51,22 & $<0,0005$ \\
\hline $\begin{array}{c}\text { PFC graue } \\
\text { Substanz }\end{array}$ & $0,5 \%$ & $-14,4 \%$ & $-10,0 \%$ & $-15,4 \%$ & 1,115 & 15,20 & $<0.0005$ \\
\hline $\begin{array}{c}\text { PFC weiße } \\
\text { Substanz }\end{array}$ & $-12,3 \%$ & $-20,1 \%$ & $-21,7 \%$ & $-21,8 \%$ & 1,115 & 52,85 & $<0.0005$ \\
\hline $\begin{array}{c}\text { OFC graue } \\
\text { Substanz }\end{array}$ & $1,7 \%$ & $-20,4 \%$ & $-10,1 \%$ & $-15,4 \%$ & 1,115 & 12,79 & 0,001 \\
\hline $\begin{array}{c}\text { OFC weiße } \\
\text { Substanz }\end{array}$ & $-8,7 \%$ & $-13,3 \%$ & $-19,7 \%$ & $-27,6 \%$ & 1,115 & 11,15 & 0,001 \\
\hline \begin{tabular}{c} 
OFC total \\
\hline
\end{tabular} & $-1,0 \%$ & $-18,8 \%$ & $-12,4 \%$ & $-18,6 \%$ & 1,115 & 14,71 \\
\hline
\end{tabular}

Zweifaktorielle ANOVA; Legende: ${ }^{\text {a }}$ : Prozentuale Differenz weiblich versus männlich; m: männlich, w: weiblich; df: Freiheitsgrade, F: F- Statistik, p: Fehlerwahrscheinlichkeit der ersten Art

In der Kontrollgruppe korrelierte das Alter signifikant negativ mit dem Volumen der grauen Substanz des ganzen Kortex $(p=0,001)$, des Präfrontalkortex $(p=0,001)$, dem linken $(p=0,002)$ und rechten $(p=0,002)$ totalen orbitofrontalen Volumen. Bei den Patienten der drei Diagnosegruppen hingegen ließen sich nur negative Korrelationen zwischen Alter und dem gesamten Kortex bzw. dem Präfrontalkortex darstellen. Für den orbitofrontalen Kortex selbst konnte bei steigendem Alter keine signifikante Abnahme des Volumens gefunden werden. Korrelationen zwischen Alter und orbitofrontaler Asymmetrie konnten nur für die schizophrenen Patienten gezeigt werden mit $r=0,335$ und $p=0,05$ (siehe Tabelle 5).

Tabelle 5: Bivariate Korrelation nach Pearson zwischen Alter und den abhängigen Variablen

\begin{tabular}{|c|c|c|c|c|c|c|}
\hline & & Total & Kontrollen & Sz. & Bipolar & OCD \\
\hline \multirow{3}{*}{$\begin{array}{c}\text { Totale graue } \\
\text { Substanz }\end{array}$} & $r$ & $\mathbf{- 0 . 4 3 0}$ & $-\mathbf{0 . 4 5 3}$ & $-\mathbf{0 . 4 0 4}$ & $\mathbf{- 0 . 4 2 7}$ & $-0,33$ \\
\cline { 2 - 7 } & $p$ & $\mathbf{0 , 0 0 1}$ & $\mathbf{0 , 0 0 5}$ & $\mathbf{0 , 0 1 5}$ & $\mathbf{0 , 0 0 8}$ & 0,10 \\
\cline { 2 - 7 } & $n$ & $\mathbf{1 3 8}$ & $\mathbf{3 7}$ & $\mathbf{3 6}$ & $\mathbf{3 8}$ & 27 \\
\hline \multirow{2}{*}{$\begin{array}{c}\text { Totale weiße } \\
\text { Substanz }\end{array}$} & $r$ & $-0,09$ & $-0,03$ & $-0,10$ & 0,00 & $-0,12$ \\
\cline { 2 - 7 } & $p$ & 0,30 & 0,88 & 0,56 & 0,99 & 0,55 \\
\cline { 2 - 7 } & $n$ & 138 & 37 & 36 & 38 & 27 \\
\hline
\end{tabular}




\begin{tabular}{|c|c|c|c|c|c|c|}
\hline & & Total & Kontrollen & Sz. & Bipolar & $O C D$ \\
\hline \multirow[t]{3}{*}{ PFC graue Substanz ${ }^{I}$} & $r$ & -0.492 & -0.578 & -0.465 & -0.546 & -0.455 \\
\hline & $p$ & 0,001 & 0,001 & 0,006 & 0,003 & 0,019 \\
\hline & $n$ & 124 & 37 & 33 & 28 & 26 \\
\hline \multirow[t]{3}{*}{ PFC weiße Substanz ${ }^{1}$} & $r$ & -0.207 & $-0,16$ & $-0,34$ & $-0,16$ & $-0,22$ \\
\hline & $p$ & 0,021 & 0,35 & 0,052 & 0,43 & 0,27 \\
\hline & $n$ & 124 & 37 & 33 & 28 & 26 \\
\hline \multirow[t]{3}{*}{ OFC links total ${ }^{2}$} & $r$ & -0.258 & -0.369 & $-0,26$ & 0,01 & $-0,37$ \\
\hline & $p$ & 0,0020 & 0,025 & 0,12 & 0,93 & 0,06 \\
\hline & $n$ & 139 & 37 & 36 & 39 & 27 \\
\hline \multirow[t]{3}{*}{ OFC rechts total ${ }^{2}$} & $r$ & -0.266 & -0.409 & $-0,26$ & $-0,04$ & $-0,12$ \\
\hline & $p$ & 0,0020 & 0,012 & 0,12 & 0,832 & 0,56 \\
\hline & $n$ & 139 & 37 & 36 & 39 & 27 \\
\hline \multirow[t]{3}{*}{ OFC total ${ }^{2}$} & $r$ & -0.314 & -0.460 & $-0,32$ & $-0,01$ & $-0,30$ \\
\hline & $p$ & 0,00 & 0,00 & 0,055 & 0,93 & 0,12 \\
\hline & $n$ & 139 & 37 & 36 & 39 & 27 \\
\hline \multirow[t]{3}{*}{ Asymm.-Koeff. grau } & $r$ & 0,04 & $-0,13$ & 0.335 & 0,07 & 0,38 \\
\hline & $p$ & 0,61 & 0,43 & 0,05 & 0,67 & 0,05 \\
\hline & $n$ & 139 & 37 & 36 & 39 & 27 \\
\hline \multirow[t]{3}{*}{ Asymm.-Koeff. weiß } & $r$ & $-0,01$ & $-0,01$ & $-0,15$ & $-0,04$ & 0,26 \\
\hline & $p$ & 0,95 & 0,95 & 0,39 & 0,83 & 0,20 \\
\hline & $n$ & 139 & 37 & 36 & 39 & 27 \\
\hline \multirow[t]{3}{*}{ Asymm.-Koeff. Total } & $r$ & 0,00 & $-0,05$ & $-0,05$ & $-0,03$ & 0,31 \\
\hline & $p$ & 0,97 & 0,76 & 0,79 & 0,85 & 0,11 \\
\hline & $n$ & 139 & 37 & 36 & 39 & 27 \\
\hline
\end{tabular}

Legende: ${ }^{1}$ : in $\mathrm{cm}^{3}$; ${ }^{2}$ : in $\mathrm{mm}^{3}$; Sz: Schizophrene; Asymm.-Koeff.: Asymmetrie - Koeffizient $=\left(2^{\star}(\mathrm{r}-\mathrm{I}) /(\mathrm{r}+\mathrm{I})\right)$.

In der Kontrollgruppe korrelierte die Bildungsdauer signifikant positiv mit dem Volumen der totalen grauen Substanz $(p=0,001)$ und dem präfrontalen grauen Volumen $(p=0,02)$. Bei den Patienten der drei Diagnosegruppen Schizophrenie, Bipolare Störung und Zwangsstörung konnten allseits keine signifikanten Korrelationen zwischen Bildungsdauer und den Zielvolumina gezeigt werden (siehe Tabelle 6). 
Tabelle 6: Bivariate Korrelation nach Pearson zwischen Bildungsdauer und den abhängigen Variablen

\begin{tabular}{|c|c|c|c|c|c|c|}
\hline & & Total & Kontrollen & Sz. & Bipolar & $O C D$ \\
\hline \multirow{3}{*}{$\begin{array}{l}\text { Totale graue } \\
\text { Substanz }^{1}\end{array}$} & $r$ & 0,228 & 0,540 & $-0,14$ & 0.429 & 0,15 \\
\hline & $p$ & 0,0070 & 0,001 & 0,41 & 0,007 & 0,44 \\
\hline & $n$ & 137 & 36 & 36 & 38 & 27 \\
\hline \multirow{3}{*}{$\begin{array}{c}\text { Totale weiße Substanz } \\
1\end{array}$} & $r$ & $0,193^{*}$ & 0,23 & $-0,12$ & $.456^{\star \star}$ & 0,435 \\
\hline & $p$ & 0,02 & 0,18 & 0,50 & 0,004 & 0,02 \\
\hline & $n$ & 137 & 36 & 36 & 38 & 27 \\
\hline \multirow[t]{3}{*}{ PFC graue Substanz ${ }^{1}$} & $r$ & 0,15 & 0,386 & $-0,22$ & 0,23 & 0,27 \\
\hline & $p$ & 0,11 & 0,020 & 0,22 & 0,240 & 0,18 \\
\hline & $n$ & 123 & 36 & 33 & 28 & 26 \\
\hline \multirow[t]{3}{*}{ PFC weiße Substanz ${ }^{1}$} & $r$ & 0,05 & 0,05 & $-0,24$ & 0,28 & 0,25 \\
\hline & $p$ & 0,57 & 0,76 & 0,17 & 0,15 & 0,22 \\
\hline & $n$ & 123 & 36 & 33 & 28 & 26 \\
\hline \multirow[t]{3}{*}{ OFC links grau $^{2}$} & $r$ & 0,12 & 0,25 & $-0,04$ & 0,14 & 0,10 \\
\hline & $p$ & 0,17 & 0,14 & 0,84 & 0,41 & 0,61 \\
\hline & $n$ & 138 & 36 & 36 & 39 & 27 \\
\hline \multirow[t]{3}{*}{ OFC rechts grau ${ }^{2}$} & $r$ & 0,08 & 0,31 & $-0,23$ & 0,24 & 0,04 \\
\hline & $p$ & 0,37 & 0,069 & 0,18 & 0,139 & 0,86 \\
\hline & $n$ & 138 & 36 & 36 & 39 & 27 \\
\hline \multirow[t]{3}{*}{ OFC total ${ }^{2}$} & $r$ & 0,13 & 0,28 & $-0,17$ & 0,27 & 0,12 \\
\hline & $p$ & 0,13 & 0,10 & 0,34 & 0,10 & 0,55 \\
\hline & $n$ & 138 & 36 & 36 & 39 & 27 \\
\hline \multirow[t]{3}{*}{ Asymm.-Koeff. grau } & $r$ & $-0,06$ & $-0,13$ & 0,13 & $-0,18$ & $-0,11$ \\
\hline & $p$ & 0,47 & 0,44 & 0,44 & 0,28 & 0,59 \\
\hline & $n$ & 138 & 36 & 36 & 39 & 27 \\
\hline \multirow[t]{3}{*}{ Asymm.-Koeff. weiß } & $r$ & $-0,05$ & $-0,02$ & $-0,18$ & 0,09 & $-0,08$ \\
\hline & $p$ & 0,59 & 0,89 & 0,29 & 0,58 & 0,69 \\
\hline & $n$ & 138 & 36 & 36 & 39 & 27 \\
\hline \multirow[t]{3}{*}{ Asymm.-Koeff. Total } & $r$ & $-0,06$ & $-0,05$ & $-0,13$ & 0,03 & $-0,10$ \\
\hline & $p$ & 0,52 & 0,78 & 0,47 & 0,86 & 0,64 \\
\hline & $n$ & 138 & 36 & 36 & 39 & 27 \\
\hline
\end{tabular}

Legende: ${ }^{1}$ : in $\mathrm{cm}^{3} ;{ }^{2}$ : in $\mathrm{mm}^{3}$; Sz: Schizophrene; Asymm.-Koeff.: Asymmetrie- Koeffizient $=\left(2^{\star}(\mathrm{r}-\mathrm{I}) /(\mathrm{r}+\mathrm{I})\right)$.

Es ergaben sich keine signifikanten Einflüsse oder Trends des Faktors Händigkeit auf die Volumina des Gehirns (siehe Tabelle 7). 
Tabelle 7: Bivariate Korrelation nach Pearson zwischen Händigkeit und den abhängigen Variablen

\begin{tabular}{|c|c|c|c|c|c|c|c|}
\hline (2) - & $\begin{array}{l}\text { Kontrollen } \\
(r=30 ; l=7)^{a}\end{array}$ & $\begin{array}{l}\text { Schizophrene } \\
(r=27 ; \mathrm{l}=6)^{\mathrm{a}}\end{array}$ & $\begin{array}{c}\text { Bipolare } \\
(r=26 ; \mathrm{l}=1)^{\mathrm{a}}\end{array}$ & $\begin{array}{c}\text { OCD } \\
(r=23 ; \mid=3)^{a}\end{array}$ & $\mathrm{df}$ & $\mathrm{F}$ & $\mathrm{P}$ \\
\hline $\begin{array}{c}\text { Totale graue } \\
\text { Substanz }\end{array}$ & $-1,9 \%$ & $-3,0 \%$ & $23,3 \%$ & $4,3 \%$ & 1,115 & 2,01 & 0,16 \\
\hline $\begin{array}{c}\text { Totale weiße } \\
\text { Substanz }\end{array}$ & $1,6 \%$ & $1,1 \%$ & $14,7 \%$ & $-2,6 \%$ & 1,115 & 1,73 & 0,19 \\
\hline $\begin{array}{c}\text { PFC graue } \\
\text { Substanz }\end{array}$ & $-4,0 \%$ & $-6,3 \%$ & $38,2 \%$ & $3,1 \%$ & 1,115 & 1,37 & 0,24 \\
\hline $\begin{array}{c}\text { PFC weiße } \\
\text { Substanz }\end{array}$ & $0,9 \%$ & $-3,6 \%$ & $33,5 \%$ & $4,3 \%$ & 1,115 & 1,74 & 0,19 \\
\hline $\begin{array}{c}\text { OFC links } \\
\text { total }\end{array}$ & $4,3 \%$ & $-12,2 \%$ & $41,5 \%$ & $2,8 \%$ & 1,115 & 1,18 & 0,28 \\
\hline $\begin{array}{c}\text { OFC rechts } \\
\text { total }\end{array}$ & $-12,4 \%$ & $-2,0 \%$ & $11,0 \%$ & $-4,7 \%$ & 1,115 & 0,09 & 0,76 \\
\hline OFC total & $-3,8 \%$ & $-5,8 \%$ & $26,2 \%$ & $-1,2 \%$ & 1,115 & 0,16 & 0,69 \\
\hline
\end{tabular}

Zweifaktorielle ANOVA; Legende: ${ }^{\text {a }}$ : Prozentuale Differenz Linkshändig versus Rechtshändig; I: Linkshändig, r: rechtshändig; df: Freiheitsgrade, F: F- Statistik, p: Fehlerwahrscheinlichkeit der ersten Art.

Aufgrund dieser vorbereitenden Analysen in Bezug auf die intervenierenden Variablen wurden die folgenden Auswertungen der Subgruppen und der Diagnose- übergreifenden Analysen teilweise adjustiert für Alter, Bildungsdauer und Geschlecht durchgeführt.

\subsubsection{Subgruppenanalyse}

\subsubsection{Schizophrenie}

Da sich, wie im vorangehenden Teil beschrieben, zwischen den schizophrenen Patienten und den Kontrollpersonen signifikante Unterschiede einiger intervenierender Variablen ergeben haben, wurde für die folgende Subgruppenanalyse ein weitestgehend angeglichenes Sample bezüglich Alter, Geschlecht und Bildungsstand bestehend aus 23 Kontrollpersonen und 23 schizophren Ersterkrankten gebildet. Alle Differenzen waren nach diesem Matching nicht mehr signifikant (siehe Tabelle 8). 
Tabelle 8: Soziodemographische Daten gematchtes Sample Schizophrenie

\begin{tabular}{|c|c|c|c|c|c|}
\hline & Kontrollen $(N=23)^{a}$ & $\begin{array}{l}\text { Schizophrenie } \\
\qquad(N=23)^{a}\end{array}$ & $F$ & $d f$ & $p$ \\
\hline Alter ${ }^{1,3}$ & $33,51 \pm 10,91$ & $30,66 \pm 7,39$ & 1,08 & 1,44 & 0,31 \\
\hline Ausbildung $^{1,3}$ & $14,80 \pm 2,70$ & $13,78 \pm 2,83$ & 1,57 & 1,44 & 0,22 \\
\hline & & & $\mathrm{Chi}^{2}$ & $d f$ & $p$ \\
\hline Geschlecht (m:w) ${ }^{2}$ & 10:13 & $11: 12$ & 0,09 & 1 & 0,77 \\
\hline Händigkeit (r:l) ${ }^{2}$ & $17: 6$ & $17: 6$ & 0,090 & 1 & 1,00 \\
\hline $\begin{array}{l}\text { Schulabschluss } \\
(H S: R S: A B: S T)^{2}\end{array}$ & $3: 6: 9: 5$ & $4: 4: 6: 3$ & 0,76 & 3 & 0,86 \\
\hline
\end{tabular}

Legende: ${ }^{\text {a: }}$ Mittelwert und Standardabweichung, sofern nicht anders angegeben; HS: Hauptschule; RS: Realschule; AB: Abitur; ST: Studium; ${ }^{1}$ : ANOVA Test; ${ }^{2}$ : Pearson Chi²-Test (2-seitig) ; ${ }^{3}$ : in Jahren; df: Freiheitsgrade, F: F- Statistik, p: Fehlerwahrscheinlichkeit der ersten Art.

Im Vergleich zwischen den schizophren Erkrankten und den gematchten Kontrollpersonen ergaben sich keine signifikanten Unterschiede in Bezug auf die Volumina des orbitofrontalen Kortex. Es ergab sich lediglich ein Trend in Form einer-4,9\%igen Reduktion des Volumens der grauen Substanz des OFC relativ zum gesamten grauen Volumen des Cortex, $p=0,093$. Außerdem ein Trend zu einer -3,7\%igen Reduktion des absoluten rechten orbitofrontalen Kortex, $p=0,20$. Sowohl bei den Kontrollpersonen als auch bei den schizophrenen Patienten ließ sich eine Asymmetrie des orbitofrontalen Kortex zugunsten der rechten Seite zeigen mit einem Asymmetrie - Koeffizienten von 0,134 $\pm 0,28$ für die Kontrollpersonen und 0,110 \pm 0,281 für die schizophren Erkrankten (siehe Tabelle 9).

Tabelle 9: Volumina schizophrene Patienten und Kontrollpersonen

\begin{tabular}{|c|c|c|c|c|c|c|}
\hline & Kontrollen $(N=23)^{a}$ & Schizophrene $(N=23)^{a}$ & $\begin{array}{c}\text { Diff. } \\
\%\end{array}$ & $d F$ & $F$ & $p$ \\
\hline Totale graue Substanz $^{1}$ & $728,23 \pm 93,11$ & $741,49 \pm 66,10$ & 1,8 & 1,40 & 0,01 & 0,95 \\
\hline Totale weiße Substanz $^{I}$ & $433,75 \pm 0,54$ & $431,12 \pm 46,42$ & $-0,6$ & 1,40 & 0,35 & 0,56 \\
\hline PFC graue Substanz ${ }^{1}$ & $90,34 \pm 12,82$ & $86,69 \pm 11,74$ & $-4,0$ & 1,40 & 2,60 & 0,12 \\
\hline PFC weiße Substanz ${ }^{1}$ & $45,04 \pm 7,60$ & $42,91 \pm 6,74$ & $-4,7$ & 1,40 & 2,35 & 0,13 \\
\hline OFC links grau $^{2}$ & $8305,5 \pm 2403,6$ & $7995,2 \pm 1594,4$ & $-3,7$ & 1,40 & 0,95 & 0,34 \\
\hline OFC links weiß ${ }^{2}$ & $2464,3 \pm 758,8$ & $2392,2 \pm 577,8$ & $-2,9$ & 1,40 & 0,55 & 0,47 \\
\hline OFC links total ${ }^{2}$ & $10769,8 \pm 2949,8$ & $10387,4 \pm 1999,7$ & $-3,6$ & 1,40 & 0,98 & 0,33 \\
\hline OFC rechts grau ${ }^{2}$ & $9082,5 \pm 1634,3$ & $8759,1 \pm 2520,6$ & $-3,6$ & 1,40 & 1,95 & 0,17 \\
\hline OFC rechts weiß ${ }^{2}$ & $2824,2 \pm 818,8$ & $2703,0 \pm 823,5$ & $-4,3$ & 1,40 & 0,30 & 0,59 \\
\hline
\end{tabular}




\begin{tabular}{|c|c|c|c|c|c|c|}
\hline & Kontrollen $(N=23)^{a}$ & Schizophrene $(N=23)^{2}$ & $\begin{array}{c}\text { Diff. } \\
\%\end{array}$ & $d F$ & $F$ & $p$ \\
\hline OFC rechts total $^{2}$ & $11906,7 \pm 2083,3$ & $11462,1 \pm 3036,5$ & $-3,7$ & 1,40 & 1,68 & 0,20 \\
\hline OFC grau $^{2}$ & $17388,0 \pm 3570,4$ & $16754,3 \pm 3164,8$ & $-3,6$ & 1,40 & 2,54 & 0,12 \\
\hline OFC weiß ${ }^{2}$ & $5288,5 \pm 1341,7$ & $5095,3 \pm 1199,5$ & $-3,7$ & 1,40 & 0,56 & 0,46 \\
\hline OFC total $^{2}$ & $22676,5 \pm 4459,2$ & $21849,6 \pm 3985,5$ & $-3,6$ & 1,40 & 2,13 & 0,15 \\
\hline $\begin{array}{c}\text { OFC links total / } \\
\text { (TGS+TWS) }\end{array}$ & $0,0092 \pm 0,0019$ & $0,0089 \pm 0,0015$ & $-3,4$ & 1,40 & 0,66 & 0,42 \\
\hline $\begin{array}{l}\text { OFC rechts total / } \\
\text { (TGS+TWS) }\end{array}$ & $0,0102 \pm 0,0011$ & $0,0098 \pm 0,0024$ & $-4,4$ & 1,40 & 1,35 & 0,25 \\
\hline OFC grau / TGS & $0,0237 \pm 0,0029$ & $0,0226 \pm 0,0036$ & $-4,9$ & 1,40 & 2,96 & 0,093 \\
\hline $\begin{array}{l}\text { OFC links total / } \\
\text { PFC-(GS+WS) }\end{array}$ & $0,1645 \pm 0,0332$ & $0,1674 \pm 0,029$ & 1,8 & 1,40 & 0,01 & 0,92 \\
\hline $\begin{array}{l}\text { OFC rechts total / } \\
\text { PFC-(GS +WS) }\end{array}$ & $0,1697 \pm 0,0182$ & $0,1705 \pm 0,0364$ & 0,5 & 1,40 & 0,02 & 0,89 \\
\hline Asymm.-Koeff. grau & $0,134 \pm 0,280$ & $0,110 \pm 0,281$ & - & 1,40 & 0,04 & 0,85 \\
\hline Asymm.-Koeff. weiß & $0,111 \pm 0,228$ & $0,071 \pm 0,312$ & - & 1,40 & 0,35 & 0,56 \\
\hline Asymm.-Koeff. Total & $0,119 \pm 0,206$ & $0,083 \pm 0,280$ & - & 1,40 & 0,21 & 0,65 \\
\hline
\end{tabular}

MANCOVA adjustiert für Geschlecht, Alter und Ausbildung; Legende: ${ }^{\text {a: }}$ Mittelwert und Standardabweichung; ${ }^{1}$ : in ccm; ${ }^{2}$ : in cmm; TGS: Totale graue Substanz; TWS: Totale weiße Substanz; GS: Graue Substanz; WS: Weiße Substanz; Asymm.-Koeff.: Asymmetrie - Koeffizient $=\left(2^{*}(r-l) /(r+l)\right)$; df: Freiheitsgrade, F: F- Statistik, p: Fehlerwahrscheinlichkeit der ersten Art.

Bezogen auf alle schizophren Ersterkrankten der Gesamtstichprobe ergaben sich einige signifikante Reduktionen der absoluten orbitofrontalen Volumina bei Patienten mit schizophrenen Familienangehörigen (erst- und zweitgradig). Das Gesamtvolumen des orbitofrontalen Kortex war im Mittel um 7,8\% reduziert, diese Reduktion ist mit $p=0,045$ signifikant. Das totale Volumen des rechten orbitofrontalen Kortex war um 12,6\% reduziert, $p=0,018$. Die rechte weiße Substanz des orbitofrontalen Kortex zeigte sich um 19,3\% reduziert, $p=0,048$. Weiterhin zeigten sich Trends zur Reduktion des orbitofrontalen Kortex im Bezug zum Gesamthirnvolumen. Für die Asymmetrie - Koeffizienten konnten keine relevanten Veränderungen gezeigt werden (siehe Tabelle 10). 
Tabelle 10: Volumina schizophrene Patienten in Abhängigkeit der Familienanamnese

\begin{tabular}{|c|c|c|c|c|c|c|}
\hline & $\begin{array}{c}\text { Sz in Familie : nein } \\
(N=26)^{a}\end{array}$ & $\begin{array}{l}\text { Sz in Familie :ja } \\
\quad(N=10)^{a}\end{array}$ & $\begin{array}{c}\text { Diff. } \\
\%\end{array}$ & $d F$ & $F$ & $p$ \\
\hline Totale graue Substanz ${ }^{1}$ & $761,0 \pm 82,6$ & $746,1 \pm 63,2$ & $-2,0$ & 1,30 & 0,80 & 0,38 \\
\hline Totale weiße Substanz $^{1}$ & $452,9 \pm 59,9$ & $426,8 \pm 44,1$ & $-5,8$ & 1,30 & 2,48 & 0,13 \\
\hline PFC graue Substanz ${ }^{I}$ & $91,1 \pm 14,2^{b}$ & $90,8 \pm 14,5^{c}$ & $-0,3$ & 1,27 & 1,02 & 0,32 \\
\hline PFC weiße Substanz ${ }^{1}$ & $46,8 \pm 9,4^{b}$ & $44,1 \pm 7,6^{\mathrm{C}}$ & $-5,7$ & 1,27 & 2,95 & 0,10 \\
\hline OFC links grau $^{2}$ & $8553,1 \pm 1839,7$ & $8455,8 \pm 1876,4$ & $-1,1$ & 1,30 & 0,10 & 0,75 \\
\hline OFC links weiß ${ }^{2}$ & $2587,5 \pm 702,9$ & $2414,7 \pm 434,1$ & $-6,7$ & 1,30 & 1,20 & 0,28 \\
\hline OFC links total $^{2}$ & $11140,6 \pm 2387,7$ & $10870,5 \pm 2083,4$ & $-2,4$ & 1,30 & 0,33 & 0,57 \\
\hline OFC rechts grau $^{2}$ & $9473,3 \pm 2444,3$ & $8480,8 \pm 2012,4$ & $-10,5$ & 1,30 & 5,26 & 0,029 \\
\hline OFC rechts weiß ${ }^{2}$ & $2932,7 \pm 869,7$ & $2367,1 \pm 651,6$ & $-19,3$ & 1,30 & 4,24 & 0,048 \\
\hline OFC rechts total ${ }^{2}$ & $12406,0 \pm 2993,8$ & $10847,9 \pm 2350,0$ & $-12,6$ & 1,30 & 6,26 & 0,018 \\
\hline OFC grau ${ }^{2}$ & $18026,4 \pm 3186,5$ & $16936,6 \pm 3462,9$ & $-6,0$ & 1,30 & 3,77 & 0,062 \\
\hline OFC weiß ${ }^{2}$ & $5520,2 \pm 1376,5$ & $4781,8 \pm 954,8$ & $-13,4$ & 1,30 & 3,39 & 0,075 \\
\hline OFC total ${ }^{2}$ & $23546,6 \pm 4215,6$ & $21718,4 \pm 4042,9$ & $-7,8$ & 1,30 & 4,36 & 0,045 \\
\hline OFC left total / (TGS+TWS) & $0,0092 \pm 0,0018^{b}$ & $0,0092 \pm 0,0012^{c}$ & 0,3 & 1,30 & 0,01 & 0,93 \\
\hline $\begin{array}{l}\text { OFC right total / } \\
\text { (TGS+TWS) }\end{array}$ & $0,0102 \pm 0,0021^{b}$ & $0,0092 \pm 0,0017^{c}$ & $-9,6$ & 1,30 & 3,75 & 0,062 \\
\hline $\begin{array}{c}\text { OFC grau / } \\
\text { TGS }\end{array}$ & $0,0237 \pm 0,0033$ & $0,0226 \pm 0,0036$ & $-4,7$ & 2,30 & 2,54 & 0,12 \\
\hline $\begin{array}{l}\text { OFC links total / } \\
\text { PFC-(GS+WS) }\end{array}$ & $0,167 \pm 0,028$ & $0,161 \pm 0,022$ & $-3,6$ & 1,27 & 0,21 & 0,65 \\
\hline $\begin{array}{l}\text { OFC rechts total / } \\
\text { PFC-(GS +WS) }\end{array}$ & $0,172 \pm 0,035$ & $0,154 \pm 0,018$ & $-10,7$ & 1,27 & 2,37 & 0,14 \\
\hline Asymm.-Koeff. Grau & $0,119 \pm 0,275$ & $-0,035 \pm 0,219$ & - & 1,30 & 1,58 & 0,22 \\
\hline Asymm.-Koeff. weiß & $0,088 \pm 0,315$ & $0,000 \pm 0,200$ & - & 1,30 & 1,62 & 0,21 \\
\hline Asymm.-Koeff. Total & $0,099 \pm 0,283$ & $-0,007 \pm 0,168$ & - & 1,30 & 1,97 & 0,17 \\
\hline
\end{tabular}

MANCOVA adjustiert für Geschlecht, Alter und Ausbildung; Legende: Sz: Schizophrene; a: Mittelwert und Standardabweichung; ${ }^{1}$ : in $\mathrm{cm} 3 ;{ }^{2}$ : in $\mathrm{mm} 3 ;{ }^{\mathrm{b}}: \mathrm{N}=24 ;{ }^{\mathrm{c}}: \mathrm{N}=9$; TGS: Totale graue Substanz; TWS: Totale weiße Substanz; GS: Graue Substanz; WS: Weiße Substanz; Asymm-Koeff.: Asymmetrie - Koeffizient $=(2 *(r-$ I)/(r+l)); df: Freiheitsgrade, F: F- Statistik, p: Fehlerwahrscheinlichkeit der ersten Art

Abbildung 7 und 8 zeigen eine grafische Darstellung der in Tabelle 10 dargestellten Werte für das absolute und relative OFC Volumen bei Patienten mit und ohne positive Familienanamnese für Schizophrenie sowie den gesunden Kontrollpersonen. 


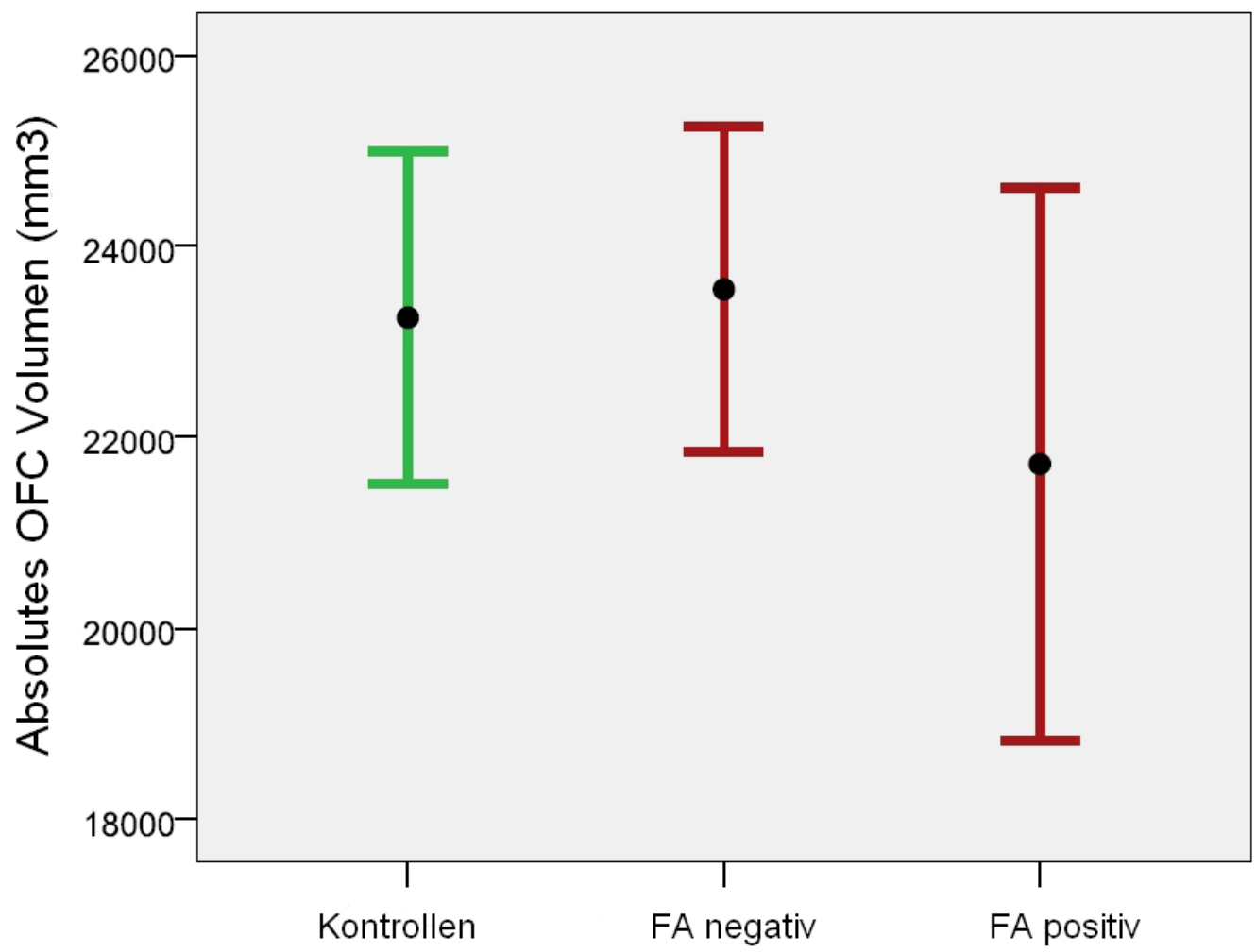

Abbildung 7 :Totales rechtes OFC Volumen bei Kontrollpersonen sowie schizophren Erkrankten mit negativer und positiver Familienanamnese

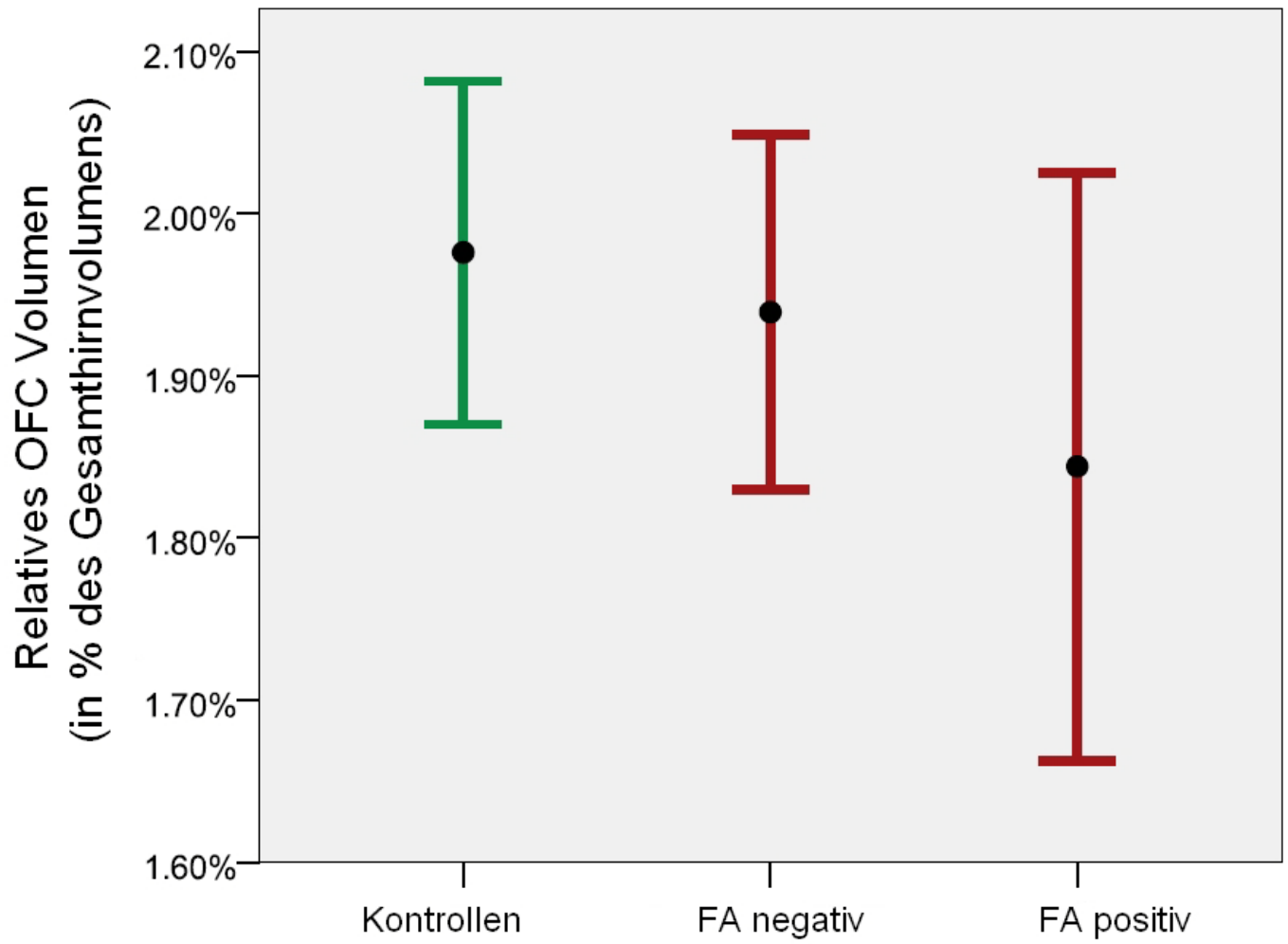

Abbildung 8 :Relatives OFC Volumen in Prozent des gesamten Hirnvolumens bei Kontrollpersonen sowie schizophren Erkrankten mit negativer und positiver Familienanamnese 
Weiterhin wurden bei den schizophrenen Patienten die Einflüsse von Dauer der unbehandelten Psychose bzw. des Prodroms, der Ausprägung im PANSS, CGI, GAF, MMSE und die Höhe der CPZ Tagesdosis auf die Zielvolumina überprüft.

Hierbei konnten signifikante positive Korrelationen zwischen der CPZ Tagesdosis und dem absoluten Volumen der linken weißen Substanz des orbitofrontalen Kortex mit $p=0,022$ gezeigt werden. Ebenfalls positiv korrelieren höhere CPZ Tagesdosen mit dem Gesamtvolumen des linken OFC relativ zum Präfrontalkortex sowie zum Gesamtkortex ( $p=0,019$ bzw. $p=0,036)$.

Darüber hinaus zeigte sich eine negative Korrelation zwischen der CPZ Tagesdosis und dem Asymmetrie - Koeffizienten für die weiße Substanz $(p=0,016)$ und für das totale orbitofrontale Volumen $(p=0,012)$. Für alle anderen Faktoren konnten keine signifikanten Volumenalterationen der Zielgrößen gefunden werden, siehe Tabelle 11.

Tabelle 11: Partielle Korrelation schizophrene Patienten und verschiedene Faktoren

\begin{tabular}{|c|c|c|c|c|c|c|c|c|}
\hline & & $\begin{array}{c}\text { Psychose- } \\
\text { dauer }^{a}\end{array}$ & $\begin{array}{c}\text { Dauer } \\
\text { Prodromi }^{a}\end{array}$ & $\begin{array}{c}\text { PANSS } \\
\text { total }\end{array}$ & CGI & GAF & MMS & $C Z P^{b}$ \\
\hline \multirow{3}{*}{$\begin{array}{c}\text { Totale } \\
\text { graue Substanz }\end{array}$} & p.c. & $-0,07$ & 0,10 & 0,11 & 0,03 & $-0,03$ & $-0,03$ & $-0,04$ \\
\hline & $p$ & 0,72 & 0,59 & 0,53 & 0,89 & 0,88 & 0,86 & 0,83 \\
\hline & df & 31 & 31 & 31 & 30 & 30 & 31 & 31 \\
\hline \multirow{3}{*}{$\begin{array}{c}\text { Totale } \\
\text { weiße Substanz }^{1}\end{array}$} & p.c. & $-0,22$ & 0,13 & 0,17 & 0,06 & 0,02 & 0,10 & 0,04 \\
\hline & $\mathrm{p}$ & 0,23 & 0,49 & 0,35 & 0,75 & 0,90 & 0,60 & 0,83 \\
\hline & df & 31 & 31 & 31 & 30 & 30 & 31 & 31 \\
\hline \multirow[t]{3}{*}{ PFC graue Substanz ${ }^{1}$} & p.c. & $-0,04$ & $-0,04$ & 0,19 & 0,09 & $-0,16$ & 0,04 & $-0,03$ \\
\hline & $\mathrm{p}$ & 0,83 & 0,82 & 0,32 & 0,65 & 0,40 & 0,84 & 0,89 \\
\hline & df & 28 & 28 & 28 & 27 & 27 & 28 & 28 \\
\hline \multirow[t]{3}{*}{ PFC weiße Substanz } & p.c. & $-0,17$ & $-0,06$ & 0,13 & 0,01 & $-0,02$ & 0,22 & 0,00 \\
\hline & $\mathrm{p}$ & 0,37 & 0,77 & 0,49 & 0,94 & 0,93 & 0,25 & 0,99 \\
\hline & df & 28 & 28 & 28 & 27 & 27 & 28 & 28 \\
\hline \multirow[t]{3}{*}{ OFC links grau $^{2}$} & p.c. & $-0,02$ & $-0,02$ & 0,19 & 0,13 & $-0,17$ & $-0,13$ & 0,26 \\
\hline & $p$ & 0,92 & 0,93 & 0,30 & 0,48 & 0,35 & 0,46 & 0,14 \\
\hline & df & 31 & 31 & 31 & 30 & 30 & 31 & 31 \\
\hline \multirow[t]{3}{*}{ OFC links weiß ${ }^{2}$} & p.c. & $-0,11$ & $-0,01$ & 0,14 & 0,18 & $-0,14$ & $-0,03$ & 0,40 \\
\hline & $p$ & 0,56 & 0,95 & 0,44 & 0,33 & 0,44 & 0,87 & 0,022 \\
\hline & df & 31 & 31 & 31 & 30 & 30 & 31 & 31 \\
\hline
\end{tabular}




\begin{tabular}{|c|c|c|c|c|c|c|c|c|}
\hline & & $\begin{array}{c}\text { Psychose- } \\
\text { dauer }^{2}\end{array}$ & $\begin{array}{c}\text { Dauer } \\
\text { Prodromi }^{a}\end{array}$ & $\begin{array}{c}\text { PANSS } \\
\text { total }\end{array}$ & CGI & $G A F$ & MMS & $C Z P^{b}$ \\
\hline \multirow[t]{3}{*}{ OFC links total ${ }^{2}$} & p.c. & $-0,05$ & $-0,02$ & 0,19 & 0,16 & $-0,18$ & $-0,12$ & 0,32 \\
\hline & $p$ & 0,79 & 0,93 & 0,30 & 0,40 & 0,33 & 0,52 & 0,07 \\
\hline & df & 31 & 31 & 31 & 30 & 30 & 31 & 31 \\
\hline \multirow[t]{3}{*}{ OFC rechts grau ${ }^{2}$} & p.c. & 0,06 & 0,30 & $-0,04$ & $-0,17$ & 0,12 & 0,01 & $-0,30$ \\
\hline & $p$ & 0,75 & 0,085 & 0,84 & 0,35 & 0,51 & 0,94 & 0,09 \\
\hline & df & 31 & 31 & 31 & 30 & 30 & 31 & 31 \\
\hline \multirow[t]{3}{*}{ OFC rechts weiß ${ }^{2}$} & p.c. & 0,05 & 0,13 & 0,05 & 0,06 & $-0,09$ & 0,14 & $-0,01$ \\
\hline & $p$ & 0,80 & 0,48 & 0,79 & 0,75 & 0,63 & 0,44 & 0,96 \\
\hline & df & 31 & 31 & 31 & 30 & 30 & 31 & 31 \\
\hline \multirow[t]{3}{*}{ OFC rechts total ${ }^{2}$} & p.c. & 0,07 & 0,28 & $-0,01$ & $-0,11$ & 0,07 & 0,07 & $-0,24$ \\
\hline & $p$ & 0,69 & 0,11 & 0,95 & 0,54 & 0,71 & 0,71 & 0,18 \\
\hline & df & 31 & 31 & 31 & 30 & 30 & 31 & 31 \\
\hline \multirow{3}{*}{$\begin{array}{c}\text { OFC links total/ } \\
\text { (TGS+TWS) }\end{array}$} & p.c. & 0,00 & $-0,08$ & 0,11 & 0,13 & $-0,18$ & $-0,16$ & 0,37 \\
\hline & $p$ & 1,00 & 0,64 & 0,53 & 0,49 & 0,33 & 0,38 & 0,036 \\
\hline & df & 31 & 31 & 31 & 30 & 30 & 31 & 31 \\
\hline \multirow{3}{*}{$\begin{array}{c}\text { OFC rechts total / } \\
\text { (TGS+TWS) }\end{array}$} & p.c. & 0,13 & 0,27 & $-0,10$ & $-0,17$ & 0,09 & 0,05 & $-0,26$ \\
\hline & $p$ & 0,48 & 0,13 & 0,58 & 0,37 & 0,62 & 0,78 & 0,15 \\
\hline & df & 31 & 31 & 31 & 30 & 30 & 31 & 31 \\
\hline \multirow{3}{*}{$\begin{array}{l}\text { OFC links total / } \\
\text { PFC-(GS+WS) }\end{array}$} & p.c. & 0,12 & 0,09 & 0,00 & 0,03 & $-0,07$ & $-0,18$ & 0,43 \\
\hline & $p$ & 0,54 & 0,62 & 0,99 & 0,89 & 0,73 & 0,34 & 0,019 \\
\hline & df & 28 & 28 & 28 & 27 & 27 & 28 & 28 \\
\hline \multirow{3}{*}{$\begin{array}{l}\text { OFC rechts total/ } \\
\text { PFC-(GS+WS) }\end{array}$} & p.c. & 0,17 & 0,28 & $-0,17$ & $-0,12$ & 0,12 & $-0,01$ & $-0,24$ \\
\hline & $p$ & 0,38 & 0,14 & 0,36 & 0,53 & 0,55 & 0,98 & 0,21 \\
\hline & $\mathrm{df}$ & 28 & 28 & 28 & 27 & 27 & 28 & 28 \\
\hline \multirow[t]{3}{*}{ Asymm.-Koeff. grau } & p.c. & 0,13 & 0,14 & $-0,02$ & $-0,05$ & $-0,01$ & 0,16 & $-0,32$ \\
\hline & $p$ & 0,47 & 0,43 & 0,93 & 0,77 & 0,95 & 0,38 & 0,07 \\
\hline & $d f$ & 31 & 31 & 31 & 30 & 30 & 31 & 31 \\
\hline \multirow[t]{3}{*}{ Asymm.-Koeff. weiß } & p.c. & 0,08 & 0,28 & $-0,14$ & $-0,17$ & 0,18 & 0,11 & $-0,42$ \\
\hline & $p$ & 0,65 & 0,12 & 0,45 & 0,35 & 0,31 & 0,54 & 0,016 \\
\hline & $\mathrm{df}$ & 31 & 31 & 31 & 30 & 30 & 31 & 31 \\
\hline \multirow[t]{3}{*}{ Asymm.-Koeff total } & p.c. & 0,11 & 0,27 & $-0,13$ & $-0,16$ & 0,16 & 0,14 & $-0,43$ \\
\hline & $\mathrm{p}$ & 0,55 & 0,13 & 0,48 & 0,37 & 0,39 & 0,45 & 0,012 \\
\hline & df & 31 & 31 & 31 & 30 & 30 & 31 & 31 \\
\hline
\end{tabular}

Partielle Korrelation angepasst für Alter, Ausbildung und Geschlecht ; Legende: ${ }^{1}$ : in $\mathrm{cm}^{3}$; ${ }^{2}$ : in $\mathrm{mm}^{3}$; ${ }^{\text {a: }}$ in Wochen; ' : Tagesdosis; TGS: Totale graue Substanz; TWS: Totale weiße Substanz; GS: Graue Substanz; WS: Weiße Substanz; Asymm. -Koeff.: Asymmetrie - Koeffizient $=\left(2^{\star}(r-l) /(r+l)\right)$. 


\subsubsection{Bipolare Störung}

Für die bipolaren Patienten wurde ebenfalls ein Sample aus jeweils 24 Patienten erstellt, welches bezüglich der intervenierenden demografischen Faktoren keine signifikanten Unterschiede mehr aufwies (siehe Tabelle 12).

Tabelle 12: Soziodemografische Daten gematchtes Sample Bipolare

\begin{tabular}{|c|c|c|c|c|c|}
\hline & Kontrollen $(N=24)^{a}$ & Bipolare $(N=24)^{a}$ & $F$ & $d f$ & $p$ \\
\hline Alter ${ }^{1,3}$ & $36,78 \pm 9,62$ & $38,7 \pm 10,46$ & 0,30 & 1,46 & 0,59 \\
\hline Ausbildung $^{1,3}$ & $14,60 \pm 2,67$ & $14,15 \pm 2,52$ & 0,37 & 1,46 & 0,77 \\
\hline & & & $\mathrm{Chi}^{2}$ & $d f$ & $p$ \\
\hline Geschlecht (m:w) $^{2}$ & $11: 13$ & $10: 14$ & 0,09 & 1 & 0,77 \\
\hline Händigkeit (r:l) ${ }^{2}$ & $21: 3$ & $23: 1$ & 1,09 & 1 & 0,30 \\
\hline $\begin{array}{l}\text { Schulabschluss } \\
\text { (HS:RS:AB:ST) }^{2}\end{array}$ & $2: 8: 5: 9$ & $6: 4: 8: 6$ & 4,63 & 3 & 0,20 \\
\hline
\end{tabular}

Legende: a: Mittelwert und Standardabweichung, sofern nicht anders angegeben; HS: Hauptschule; RS: Realschule; AB: Abitur; ST: Studium; ${ }^{1}$ : ANOVA Test; ${ }^{2}$ : Pearson Chi²-Test (2-seitig) ; ${ }^{3}$ : in Jahren; df: Freiheitsgrade, F: F- Statistik, p: Fehlerwahrscheinlichkeit der ersten Art

Bei den bipolaren Patienten zeigte sich im Vergleich zu den Kontrollpersonen ein Trend zur Reduktion des absoluten rechten OFC- Volumens mit $-11 \%$ und $p=0,14$. Darüber hinaus fand sich eine signifikante Reduktion des rechten OFC- Volumens in Relation zum Gesamtkortex $(p=0,027)$, dies ließ sich auch isoliert für die graue $(p=0,036)$ sowie weiße Substanz $(p=0,046)$ des rechten orbitofrontalen Kortex zeigen. Bei den gematchten Kontrollpersonen ließ sich eine Asymmetrie des orbitofrontalen Kortex zugunsten der rechten Seite zeigen mit einem Asymmetrie Koeffizienten von $0,111 \pm 0,238$. Für die bipolaren Patienten war eine minimale Asymmetrie zugunsten links festzustellen mit einem Koeffizienten von -0,016 \pm 0,252 . Diese Alteration war mit $p=0,12$ allerdings nicht signifikant (siehe Tabelle 13).

Tabelle 13: Volumina Bipolare und Kontrollpersonen

\begin{tabular}{|c|c|c|c|c|c|c|}
\hline & ${\text { Kontrollen }(N=24)^{a}}$ & Bipolare $(N=24)^{a}$ & $\begin{array}{c}\text { Diff. } \\
\%\end{array}$ & $d F$ & $F$ \\
\hline Totale graue Substanz $^{1}$ & $733,5 \pm 70,9$ & $725,8 \pm 75,3$ & $-1,0$ & 1,42 & 0,19 & 0,67 \\
\hline Totale weiße Substanz $^{1}$ & $441,4 \pm 52,4$ & $431,1 \pm 51,4$ & $-2,3$ & 1,42 & 0,06 & 0,80 \\
\hline PFC graue Substanz $^{1}$ & $90,6 \pm 12,3$ & $89,5 \pm 14,1$ & $-1,2$ & 1,42 & 0,18 & 0,68 \\
\hline PFC weiße Substanz $^{1}$ & $46,5 \pm 7,7$ & $44,7 \pm 8,3$ & $-4,0$ & 1,42 & 0,14 & 0,71 \\
\hline
\end{tabular}




\begin{tabular}{|c|c|c|c|c|c|c|}
\hline OFC links grau ${ }^{2}$ & $8311 \pm 1971$ & $7947 \pm 1611$ & $-4,4$ & 1,42 & 0,16 & 0,69 \\
\hline OFC links weiß ${ }^{2}$ & $2747 \pm 1009$ & $2559 \pm 931$ & $-6,8$ & 1,42 & 0,16 & 0,69 \\
\hline OFC links total ${ }^{2}$ & $11058 \pm 2608$ & $10506 \pm 2179$ & $-5,0$ & 1,42 & 0,21 & 0,65 \\
\hline OFC rechts grau ${ }^{2}$ & $9344 \pm 2239$ & $8074 \pm 1911$ & $-13,6$ & 1,42 & 2,93 & 0,094 \\
\hline OFC rechts weiß ${ }^{2}$ & $3084 \pm 1508$ & $2333 \pm 654$ & $-24,3$ & 1,42 & 3,62 & 0,064 \\
\hline OFC rechts total ${ }^{2}$ & $12428 \pm 3591$ & $10407 \pm 2436$ & $-16,3$ & 1,42 & 3,59 & 0,065 \\
\hline OFC grau ${ }^{2}$ & $17655 \pm 3419$ & $16021 \pm 2756$ & $-9,3$ & 1,42 & 1,94 & 0,17 \\
\hline OFC weiß ${ }^{2}$ & $5831 \pm 2324$ & $4892 \pm 1467$ & $-16,1$ & 1,42 & 1,81 & 0,19 \\
\hline${\text { OFC } \text { total }^{2}}^{2}$ & $23486 \pm 5356$ & $20913 \pm 3859$ & $-11,0$ & 1,42 & 2,23 & 0,14 \\
\hline $\begin{array}{c}\text { OFC links total / } \\
\text { (TGS+TWS) }\end{array}$ & $0,009 \pm 0,002$ & $0,009 \pm 0,002$ & $-2,8$ & 1,42 & 0,20 & 0,66 \\
\hline OFC rechts grau / TGS & $0,013 \pm 0,002$ & $0,011 \pm 0,002$ & $-12,3$ & 1,42 & 4,68 & 0,036 \\
\hline OFC rechts weiß / TWS & $0,007 \pm 0,003$ & $0,005 \pm 0,001$ & $-21,7$ & 1,42 & 4,25 & 0,046 \\
\hline $\begin{array}{l}\text { OFC rechts total / } \\
\text { (TGS+TWS) }\end{array}$ & $0,010 \pm 0,002$ & $0,009 \pm 0,002$ & $-14,5$ & 1,42 & 5,23 & 0,027 \\
\hline OFC grau /TGS & $0,024 \pm 0,003$ & $0,022 \pm 0,003$ & $-7,8$ & 1,42 & 3,41 & 0,072 \\
\hline $\begin{array}{l}\text { OFC links total/ } \\
\text { PFC-(GS+WS) }\end{array}$ & $0,168 \pm 0,028$ & $0,163 \pm 0,032$ & $-3,2$ & 1,42 & 0,45 & 0,51 \\
\hline $\begin{array}{l}\text { OFC rechts total / } \\
\text { PFC-(GS +WS) }\end{array}$ & $0,173 \pm 0,036$ & $0,151 \pm 0,028$ & $-12,6$ & 1,42 & 4,60 & 0,038 \\
\hline Asymm.-Koeff. Grau & $0,091 \pm 0,298$ & $-0,076 \pm 0,243$ & - & 1,42 & 3,87 & 0,056 \\
\hline Asymm.-Koeff. Weiß & $0,120 \pm 0,261$ & $0,009 \pm 0,285$ & - & 1,42 & 1,49 & 0,23 \\
\hline Asymm.-Koeff. Total & $0,111 \pm 0,238$ & $-0,016 \pm 0,252$ & - & 1,42 & 2,51 & 0,12 \\
\hline
\end{tabular}

MANCOVA adjustiert für Geschlecht, Alter und Ausbildung ; Legende: ${ }^{a}:$ Mittelwert und Standardabweichung; ${ }^{1}$ : in $\mathrm{cm}^{3}$; ${ }^{2}$ : in $\mathrm{mm}^{3}$; TGS: Totale graue Substanz; TWS: Totale weiße Substanz; GS: Graue Substanz; WS: Weiße Substanz; Asymm. -Koeff.: Asymmetrie - Koeffizient $=(2 *(r-1) /(r+l))$; df: Freiheitsgrade, F: F- Statistik, $p$ : Fehlerwahrscheinlichkeit der ersten Art.

Bei den bipolaren Patienten, die Lithium eingenommen haben, konnte zwar eine signifikante Vergrößerung des präfrontalen Kortex gezeigt werden, allerdings konnte kein direkter Einfluss auf den orbitofrontalen Kortex nachgewiesen werden. Bei den Patienten mit der Einnahme eines anderen Stimmungsaufhellers als Lithium ergab sich eine signifikante Reduktion des absoluten linken orbitofrontalen Kortex $(p=0,043)$ sowie Trends zur Invertierung der Asymmetrie zugunsten der linken Hälfte des orbitofrontalen Kortex (siehe Tabelle 14 und 15). 
Tabelle 14: Volumina Bipolare in Abhängigkeit von Lithiumeinnahme

\begin{tabular}{|c|c|c|c|c|c|c|}
\hline 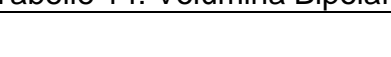 & Lithium: nein $(N=22)^{a}$ & Lithium: ja $(N=16)^{a}$ & Diff. \% & $d F$ & $F$ & $p$ \\
\hline Totale graue Substanz ${ }^{1}$ & $723,4 \pm 72,6$ & $722,761 \pm 87,1$ & $-0,1$ & 1,31 & 1,03 & 0,32 \\
\hline Totale weiße Substanz $^{1}$ & $422,2 \pm 48,7$ & $441,7 \pm 53,8$ & 4,6 & 1,31 & 3,79 & 0,061 \\
\hline PFC graue Substanz ${ }^{I}$ & $86,3 \pm 12,6^{b}$ & $90,7 \pm 16,7^{\mathrm{C}}$ & 5,0 & 1,22 & 4,70 & 0,041 \\
\hline PFC weiße Substanz ${ }^{1}$ & $41,9 \pm 8,1^{b}$ & $48,4 \pm 8,1^{c}$ & 15,4 & 1,22 & 8,83 & 0,007 \\
\hline OFC links total $^{2}$ & $10159 \pm 2032$ & $10701 \pm 2114^{d}$ & 5,3 & 1,32 & 1,02 & 0,32 \\
\hline OFC rechts total ${ }^{2}$ & $10472 \pm 2248$ & $11029 \pm 2504^{d}$ & 5,3 & 1,32 & 0,88 & 0,36 \\
\hline Asymm.-Koeff. Grau & $-0,072 \pm 0,232$ & $0,043 \pm 0,214$ & $-158,6$ & 1,28 & 1,93 & 0,18 \\
\hline Asymm.-Koeff. Weiß & $0,061 \pm 0,285$ & $0,024 \pm 0,226$ & $-60,5$ & 1,28 & 0,29 & 0,60 \\
\hline Asymm.-Koeff. Total & $0,026 \pm 0,250$ & $0,024 \pm 0,191$ & $-6,2$ & 1,28 & 0,01 & 0,91 \\
\hline
\end{tabular}

MANCOVA adjustiert für Geschlecht, Alter und Ausbildung ; Legende: ${ }^{a}$ : Mittelwert und Standardabweichung; ${ }^{1}$ : in $\mathrm{cm}^{3} ;{ }^{2}$ : in $\mathrm{mm}^{3} ;{ }^{\mathrm{b}}: \mathrm{N}=15{ }^{\mathrm{c}}: \mathrm{N}=13 ;{ }^{\mathrm{d}}: \mathrm{N}=16$; Asymm. - Koeff.: Asymmetrie - Koeffizient $=\left(2^{*}(\mathrm{r}-\mathrm{I}) /(\mathrm{r}+\mathrm{I})\right)$; df: Freiheitsgrade, F: F- Statistik, p: Fehlerwahrscheinlichkeit der ersten Art.

Tabelle 15: Volumina Bipolare in Abhängigkeit von anderen Stimmungsaufheller als Lithium

\begin{tabular}{|c|c|c|c|c|c|c|}
\hline & $\begin{array}{c}\text { Stimmungsaufheller: } \\
\text { nein }(N=12)^{a}\end{array}$ & $\begin{array}{c}\text { Stimmungsaufhelle } \\
\text { r: ja }(N=26)^{a}\end{array}$ & Diff. \% & $d F$ & $F$ & $p$ \\
\hline Totale graue Substanz ${ }^{1}$ & $749,5 \pm 85,6^{b}$ & $712,0 \pm 72,8$ & $-5,0$ & 1,31 & 1,24 & 0,27 \\
\hline Total weiße Substanz ${ }^{1}$ & $454,4 \pm 54,1^{b}$ & $419,8 \pm 47,0$ & $-7,6$ & 1,31 & 3,64 & 0,066 \\
\hline PFC graue Substanz ${ }^{1}$ & $96,7 \pm 16,8^{c}$ & $85,0 \pm 12,5^{d}$ & $-12,1$ & 1,22 & 0,87 & 0,36 \\
\hline PFC weiße Subsatnz ${ }^{I}$ & $51,2 \pm 8,6^{\mathrm{c}}$ & $42,4 \pm 7,4^{d}$ & $-17,2$ & 1,22 & 3,33 & 0,082 \\
\hline OFC links total ${ }^{2}$ & $11325 \pm 1896$ & $9954 \pm 2015$ & $-12,1$ & 1,32 & 4,45 & 0,043 \\
\hline OFC rechts total ${ }^{2}$ & $11143 \pm 2780$ & $10505 \pm 2142$ & $-5,7$ & 1,32 & 0,07 & 0,79 \\
\hline Asymm.-Koeff. Grau & $-0,069 \pm 0,234$ & $-0,004 \pm 0,228$ & $-94,8$ & 1,32 & 0,81 & 0,37 \\
\hline Asymm.-Koeff. Weiß & $-0,021 \pm 0,277$ & $0,077 \pm 0,250$ & $-457,9$ & 1,32 & 3,54 & 0,069 \\
\hline Asymm.-Koeff. Total & $-0,033 \pm 0,247$ & $0,052 \pm 0,213$ & $-257,0$ & 1,32 & 3,32 & 0,078 \\
\hline
\end{tabular}

MANCOVA angepasst für Geschlecht, Alter und Ausbildung; Legende: ${ }^{a}$ : Mittelwert und Standardabweichung; 1: in $\mathrm{cm}^{3} ; 2$ : in $\mathrm{mm}^{3} ;{ }^{b}: \mathrm{N}=11 ;^{\mathrm{c}}: \mathrm{N}=8 ;{ }^{\mathrm{d}}$ : $\mathrm{N}=20$; Asymm-Koeff.: Asymmetrie - Koeffizient $=\left(2^{\star}(\mathrm{r}-\mathrm{I}) /(\mathrm{r}+\mathrm{l})\right)$; df: Freiheitsgrade, F: F- Statistik, p: Fehlerwahrscheinlichkeit der ersten Art.

Es ergaben sich keine signifikanten Unterschiede zwischen Patienten mit und ohne psychotische Symptome. Bei den bipolaren Patienten mit psychotischen Symptomen zeigten sich lediglich Trends zur Reduktion des absoluten rechten orbitofrontalen Volumens $(-14,6 \%, p=0,068)$ und relativ zum Volumen des präfrontalen Kortex $(-11,6 \%, p=0,099)$ sowie des Gesamthirns $(-11,9 \%, p=0,089)$. Änderungen der Asymmetrie in Abhängigkeit vom Vorhandensein psychotischer Symptome konnten keine gefunden werden (siehe Tabelle 16). 
Tabelle 16: Volumina Bipolare in Abhängigkeit von Psychotischen Symptomen

\begin{tabular}{|c|c|c|c|c|c|c|}
\hline & $\begin{array}{c}\text { Psychotische Symptome: } \\
\text { nein }(N=27)^{a}\end{array}$ & $\begin{array}{c}\text { Psychotische Symptome: } \\
\text { ja }(N=11)^{a}\end{array}$ & $\begin{array}{c}\text { Diff. } \\
\%\end{array}$ & $d F$ & $F$ & $p$ \\
\hline Totale graue Substanz $^{1}$ & $735,5 \pm 83,5$ & $689,9 \pm 47,6^{c}$ & $-6,2$ & 1,31 & 2,32 & 0,14 \\
\hline Totale weiße Substanz $^{1}$ & $439,1 \pm 50,4$ & $405,9 \pm 46,6^{c}$ & $-7,6$ & 1,31 & 3,34 & 0,077 \\
\hline PFC graue Substanz ${ }^{I}$ & $89,4 \pm 16,5^{b}$ & $86,1 \pm 9,5^{d}$ & $-3,7$ & 1,22 & 0,35 & 0,56 \\
\hline PFC weiße Substanz ${ }^{1}$ & $46,0 \pm 8,3^{b}$ & $42,6 \pm 9,4^{d}$ & $-7,5$ & 1,22 & 1,95 & 0,18 \\
\hline OFC links total ${ }^{2}$ & $10530 \pm 2262$ & $10036 \pm 1469$ & $-4,7$ & 1,32 & 0,32 & 0,58 \\
\hline OFC rechts total ${ }^{2}$ & $11178 \pm 2214$ & $9550 \pm 2342$ & $-14,6$ & 1,32 & 3,57 & 0,068 \\
\hline $\begin{array}{c}\text { OFC rechts total / } \\
\text { (TGS+TWS) }\end{array}$ & $0,009 \pm 0,001$ & $0,008 \pm 0,002^{c}$ & $-11,9$ & 1,31 & 3,08 & 0,089 \\
\hline $\begin{array}{c}\text { OFC rechts total / } \\
\text { PFC-(GS+WS) }\end{array}$ & $0,157 \pm 0,026^{b}$ & $0,139 \pm 0,023^{d}$ & $-11,6$ & 1,22 & 2,97 & 0,099 \\
\hline Asymm..-Koeff. Grau & $0,013 \pm 0,197$ & $-0,114 \pm 0,283$ & - & 1,32 & 2,51 & 0,12 \\
\hline Asymm.-Koeff. Weiß & $0,084 \pm 0,227$ & $-0,049 \pm 0,317$ & - & 1,32 & 1,74 & 0,20 \\
\hline Asymm.-Koeff. Total & $0,062 \pm 0,181$ & $-0,065 \pm 0,299$ & - & 1,32 & 2,23 & 0,15 \\
\hline
\end{tabular}

MANCOVA angepasst für Alter, Ausbildung und Geschlecht; Legende: ' ${ }^{\text {: }}$ Mittelwert und Standardabweichung; 1: in cm3; 2: in mm3; ${ }^{b}: N=19 ;^{c}: N=10 ;{ }^{d}: N=9$; TGS: Totale graue Substanz; TWS: Totale weiße Substanz; GS: Graue Substanz; WS: Weiße Substanz; Asymm. -Koeff.: Asymmetrie - Koeffizient $=\left(2{ }^{\star}(r-\mathrm{l}) /(\mathrm{r}+\mathrm{l})\right)$; df: Freiheitsgrade, F: F- Statistik, p: Fehlerwahrscheinlichkeit der ersten Art.

Das Volumen der gesamten grauen Substanz war bei Patienten mit Neuroleptikaeinnahme signifikant reduziert $(p=0,027)$. Für die absoluten oder relativen Volumina sowie die Asymmetrie- Koeffizienten des orbitofrontalen Kortex ergaben sich allerdings weder Trends noch Signifikanzen (siehe Tabelle 17).

Tabelle 17: Volumina Bipolare in Abhängigkeit von Neuroleptikaeinnahme

\begin{tabular}{|c|c|c|c|c|c|c|}
\hline & $\begin{array}{l}\text { Neuroleptika: } \\
\text { nein }(N=19)^{a}\end{array}$ & $\begin{array}{l}\text { Neuroleptika: } \\
\text { KON }(N=4)^{a}\end{array}$ & $\begin{array}{l}\text { Neuroleptika: } \\
\text { ATYP }(N=14)^{a}\end{array}$ & $d F$ & $F$ & $p$ \\
\hline Totale graue Substanz $^{1}$ & $754,7 \pm 77,8$ & $662,6 \pm 45,1$ & $692,3 \pm 67,1^{d}$ & 2,29 & 4,10 & 0,027 \\
\hline Totale weiße Substanz $^{I}$ & $439,0 \pm 41,9$ & $402,6 \pm 23,9$ & $421,7 \pm 66,1^{d}$ & 2,29 & 1,22 & 0,31 \\
\hline PFC graue Substanz ${ }^{1}$ & $96,0 \pm 13,3^{b}$ & $76,5 \pm 14,0^{c}$ & $81,5 \pm 11,9$ & 2,20 & 6,38 & 0,007 \\
\hline PFC weiße Substanz ${ }^{1}$ & $47,9 \pm 6,0^{b}$ & $40,1 \pm 6,0^{c}$ & $42,3 \pm 11,6$ & 2,20 & 1,55 & 0,24 \\
\hline OFC links total ${ }^{2}$ & $10851 \pm 1897$ & $9865 \pm 1895$ & $9738 \pm 2223$ & 2,30 & 0,90 & 0,42 \\
\hline OFC rechts total ${ }^{2}$ & $11353 \pm 2053$ & $9490 \pm 2375$ & $10313 \pm 2639$ & 2,30 & 1,21 & 0,31 \\
\hline Asymm.-Koeff. Grau & $0,006 \pm 0,229$ & $-0,077 \pm 0,325$ & $-0,022 \pm 0,194$ & 2,30 & 0,78 & 0,47 \\
\hline Asymm.-Koeff. Weiß & $0,062 \pm 0,220$ & $-0,032 \pm 0,233$ & $0,074 \pm 0,310$ & 2,30 & 0,03 & 0,97 \\
\hline Asymm.-Koeff. Total & $0,042 \pm 0,182$ & $-0,045 \pm 0,249$ & $0,050 \pm 0,263$ & 2,30 & 0,03 & 0,97 \\
\hline
\end{tabular}

MANCOVA angepasst für Alter, Ausbildung und Geschlecht ; Legende: ${ }^{a}$ : Mittelwert und Standardabweichung; ${ }^{1}$ : in $\mathrm{cm}^{3} ;{ }^{2}$ : in $\mathrm{mm} 3 ;{ }^{\mathrm{b}}: \mathrm{N}=14 ;{ }^{\mathrm{c}}: \mathrm{N}=3$; ${ }^{\mathrm{d}}: \mathrm{N}=13$; KON: konventionelle Neuroleptika; ATYP: atypische Neuroleptika; Asymm. -Koeff.: Asymmetrie - Koeffizient $=(2 *(r-l) /(r+l))$; df: Freiheitsgrade, F: FStatistik, p: Fehlerwahrscheinlichkeit der ersten Art. 
Für die bipolaren Patienten ergaben sich negative Korrelationen zwischen Krankheitsdauer und absoluten rechten orbitofrontalen Volumen der weißen Substanz mit p.c. $=-0,37$ und $p=0,03$ sowie dem gesamten orbitofrontalen Volumen im Bezug zur Gesamthirnmasse mit p.c. $=-0,39$ und $p=0,023$. Das Alter bei Diagnosestellung hingegen korrelierte positiv mit dem weißen, grauen und totalen orbitofrontalen Volumen im Bezug zum Gesamthirn (grau: p.c. $=0,36$, $p=0,041$; weiß: p.c. $=0,37, p=0,035$; total: p.c. $=0,41, p=0,020)$. Weiterhin wurde die Anzahl der depressiven und manischen Episoden der Probanden auf Wechselbeziehung mit den Zielvolumina geprüft. Hier konnte eine Korrelation zwischen Anzahl der depressiven Episoden und Verminderung des rechten grauen orbitofrontalen Volumens mit p.c. $=-0,36$ und $p=0,043$ gezeigt werden Für die Anzahl der manischen Episoden ließen sich keine signifikanten Volumenveränderungen darstellen. Der Summenwert des Depressions- Scores MADRS korrelierte negativ mit dem gesamten Volumen und dem präfrontalen Volumen der grauen Substanz, es konnte jedoch kein Einfluss auf die orbitofrontalen Volumina gezeigt werden. Für den YMRS, der die klinische Ausprägung einer Manie widerspiegelt, konnten keine relevanten Volumenalterationen gezeigt werden (siehe Tabelle 18).

Tabelle 18: Partielle Korrelation Bipolare für verschiedene Faktoren

\begin{tabular}{|c|c|c|c|c|c|c|c|}
\hline & 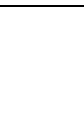 & $\begin{array}{c}\text { Krankheits- } \\
\text { dauer }\end{array}$ & $\begin{array}{l}\text { Alter bei } \\
\text { Diagnose }\end{array}$ & $\begin{array}{c}\text { Depr. } \\
\text { Episoden }\end{array}$ & $\begin{array}{c}\text { Man. } \\
\text { Episoden }\end{array}$ & MADRS & YMRS \\
\hline \multirow{3}{*}{$\begin{array}{c}\text { Totale } \\
\text { graue Substanz }{ }^{1}\end{array}$} & p.c. & 0,12 & $-0,15$ & $-0,04$ & $-0,12$ & $-0,42$ & 0,00 \\
\hline & $p$ & 0,51 & 0,42 & 0,83 & 0,53 & 0,019 & 0,99 \\
\hline & $d f$ & 32 & 30 & 29 & 29 & 29 & 28 \\
\hline \multirow{3}{*}{$\begin{array}{c}\text { Totale } \\
\text { weiße Substanz }{ }^{1}\end{array}$} & p.c. & $-0,01$ & 0,02 & $-0,13$ & $-0,12$ & 0,00 & $-0,04$ \\
\hline & $p$ & 0,97 & 0,93 & 0,48 & 0,54 & 1,00 & 0,85 \\
\hline & $d f$ & 32 & 30 & 29 & 29 & 29 & 28 \\
\hline \multirow{3}{*}{$\begin{array}{c}\text { PFC } \\
\text { graue Substanz }{ }^{1}\end{array}$} & p.c. & $-0,05$ & $-0,09$ & $-0,30$ & $-0,14$ & $-0,53$ & $-0,03$ \\
\hline & $p$ & 0,82 & 0,68 & 0,17 & 0,54 & 0,010 & 0,89 \\
\hline & $d f$ & 23 & 22 & 20 & 20 & 21 & 21 \\
\hline \multirow{3}{*}{$\begin{array}{c}\text { PFC } \\
\text { weiße Substanz }{ }^{2}\end{array}$} & p.c. & 0,02 & 0,00 & $-0,36$ & 0,05 & $-0,17$ & $-0,02$ \\
\hline & $p$ & 0,93 & 1,00 & 0,10 & 0,82 & 0,44 & 0,92 \\
\hline & $d f$ & 23 & 22 & 20 & 20 & 21 & 21 \\
\hline
\end{tabular}




\begin{tabular}{|c|c|c|c|c|c|c|c|}
\hline & & $\begin{array}{c}\text { Krankheits- } \\
\text { dauer }\end{array}$ & $\begin{array}{l}\text { Alter bei } \\
\text { Diagnose }\end{array}$ & $\begin{array}{c}\text { Depr. } \\
\text { Episoden }\end{array}$ & $\begin{array}{c}\text { Man. } \\
\text { Episoden }\end{array}$ & MADRS & YMRS \\
\hline \multirow{3}{*}{ OFC rechts grau ${ }^{2}$} & p.c. & $-0,21$ & 0,18 & $-0,36$ & $-0,14$ & $-0,17$ & $-0,07$ \\
\hline & $p$ & 0,22 & 0,32 & 0,043 & 0,46 & 0,35 & 0,71 \\
\hline & $d f$ & 33 & 31 & 30 & 30 & 30 & 29 \\
\hline \multirow{3}{*}{ OFC rechts weiß ${ }^{2}$} & p.c. & $-0,37$ & 0,35 & $-0,15$ & $-0,17$ & $-0,23$ & $-0,04$ \\
\hline & $p$ & 0,030 & 0,050 & 0,41 & 0,35 & 0,20 & 0,83 \\
\hline & $d f$ & 33 & 31 & 30 & 30 & 30 & 29 \\
\hline \multirow{3}{*}{$\begin{array}{c}\text { OFC rechts grau / } \\
\text { TGS }\end{array}$} & p.c. & $-0,32$ & 0,28 & $-0,38$ & $-0,19$ & $-0,06$ & $-0,14$ \\
\hline & $p$ & 0,066 & 0,12 & 0,033 & 0,30 & 0,77 & 0,46 \\
\hline & $d f$ & 32 & 30 & 29 & 29 & 29 & 28 \\
\hline \multirow{3}{*}{$\begin{array}{c}\text { OFC rechts weiß / } \\
\text { TWS }\end{array}$} & p.c. & $-0,41$ & 0,38 & $-0,11$ & $-0,21$ & $-0,30$ & $-0,10$ \\
\hline & $p$ & 0,015 & 0,030 & 0,56 & 0,26 & 0,11 & 0,60 \\
\hline & $d f$ & 32 & 30 & 29 & 29 & 29 & 28 \\
\hline \multirow{3}{*}{ OFC grau / TGS } & p.c. & $-0,32$ & 0,36 & $-0,29$ & $-0,06$ & 0,03 & $-0,05$ \\
\hline & $p$ & 0,062 & 0,041 & 0,11 & 0,74 & 0,88 & 0,81 \\
\hline & $d f$ & 32 & 30 & 29 & 29 & 29 & 28 \\
\hline \multirow{3}{*}{ OFC weiß / TWS } & p.c. & $-0,39$ & 0,37 & $-0,14$ & $-0,13$ & $-0,17$ & 0,04 \\
\hline & $p$ & 0,022 & 0,035 & 0,45 & 0,49 & 0,35 & 0,82 \\
\hline & $d f$ & 32 & 30 & 29 & 29 & 29 & 28 \\
\hline \multirow{3}{*}{$\begin{array}{l}\text { OFC total/ } \\
\text { (TGS+TWS) }\end{array}$} & p.c. & $-0,39$ & 0,41 & $-0,27$ & $-0,10$ & $-0,08$ & $-0,01$ \\
\hline & $p$ & 0,023 & 0,020 & 0,14 & 0,59 & 0,67 & 0,94 \\
\hline & $d f$ & 32 & 30 & 29 & 29 & 29 & 28 \\
\hline \multirow{3}{*}{ Asymm.-Koeff. Grau } & p.c. & $-0,10$ & 0,08 & 0,03 & $-0,15$ & $-0,16$ & $-0,29$ \\
\hline & $p$ & 0,59 & 0,67 & 0,87 & 0,42 & 0,40 & 0,11 \\
\hline & $d f$ & 33 & 31 & 30 & 30 & 30 & 29 \\
\hline \multirow{3}{*}{ Asymm.-Koeff. Weiß } & p.c. & $-0,11$ & 0,01 & $-0,28$ & $-0,17$ & $-0,07$ & $-0,14$ \\
\hline & $p$ & 0,53 & 0,94 & 0,13 & 0,37 & 0,69 & 0,47 \\
\hline & $d f$ & 33 & 31 & 30 & 30 & 30 & 29 \\
\hline \multirow{3}{*}{ Asymm.-Koeff. Total } & p.c. & $-0,09$ & 0,01 & $-0,23$ & $-0,17$ & $-0,12$ & $-0,19$ \\
\hline & $p$ & 0,59 & 0,97 & 0,21 & 0,34 & 0,53 & 0,30 \\
\hline & $d f$ & 33 & 31 & 30 & 30 & 30 & 29 \\
\hline
\end{tabular}

Partielle Korrelation angepasst für Alter, Ausbildung und Geschlecht ; Legende: ${ }^{1}$ : in $\mathrm{cm}^{3} ;{ }^{2}:$ in $\mathrm{mm}^{3}$ Depr. Episoden: Depressive Episoden; Man. Episoden: Manische Episoden; TGS: Totale graue Substanz; TWS: Totale weiße Substanz; Asymm. -Koeff.: Asymmetrie - Koeffizient $=\left(2^{\star}(\mathrm{r}-\mathrm{l}) /(\mathrm{r}+\mathrm{l})\right)$.

\subsubsection{Zwangsstörung}

Es wurde zunächst ein weitestgehend ausgeglichenes Sample bestehend aus 26 Kontrollpersonen und 26 Zwangspatienten gebildet. Nach dem Matching war die 
mittlere Bildungsdauer bei letzterer Gruppe noch reduziert, aber die Unterschiede bezüglich der demografischen Variablen waren nach dem Matching nicht mehr signifikant (siehe Tabelle 19).

Tabelle 19: Soziodemographische Daten gematchtes Sample Zwangsstörung

\begin{tabular}{|c|c|c|c|c|c|}
\hline & $\begin{array}{c}\text { Kontrollen } \\
(N=26)^{a}\end{array}$ & $\begin{array}{c}\text { Zwangsstörung } \\
(N=26)^{a}\end{array}$ & $F$ & $d f$ & $p$ \\
\hline Alter ${ }^{1,3}$ & $35,15 \pm 10,77$ & $34,68 \pm 9,80$ & 0,03 & 1,50 & 0,87 \\
\hline Ausbildung $^{1,3}$ & $14,33 \pm 2,58$ & $13,54 \pm 2,34$ & 1,33 & 1,50 & 0,25 \\
\hline & & & $\mathrm{Chi}^{2}$ & $d f$ & $p$ \\
\hline${\text { Geschlecht }(m: w)^{2}}^{2}$ & $12: 14$ & $12: 14$ & 0,00 & 1 & 1,00 \\
\hline Händigkeit (r:l) ${ }^{2}$ & $22: 4$ & $23: 3$ & 0,17 & 1 & 0,69 \\
\hline $\begin{array}{l}\text { Schulabschluss } \\
\text { (HS:RS:AB:ST) }^{2}\end{array}$ & $3: 8: 8: 7$ & $6: 12: 3: 5$ & 4,41 & 3 & 0,22 \\
\hline
\end{tabular}

Legende: ${ }^{a}$ : Mittelwert und Standardabweichung, sofern nicht anders angegeben; HS: Hauptschule; RS: Realschule; AB: Abitur; ST: Studium; ${ }^{1}$ : ANOVA Test; ${ }^{2}$ : Pearson Chi²-Test (2-seitig) ; ${ }^{3}$ : in Jahren; df: Freiheitsgrade, F: F- Statistik, p: Fehlerwahrscheinlichkeit der ersten Art.

Im gematchten Sample ergaben sich für die Zwangserkrankten gegenüber den Kontrollpatienten keine Trends oder signifikanten oder relevanten Unterschiede der Zielvolumina (siehe Tabelle 20).

Tabelle 20: Volumina Zwangserkrankte und Kontrollpersonen

\begin{tabular}{|c|c|c|c|c|c|c|}
\hline & $\begin{array}{l}\text { Kontrollen } \\
(N=26)^{a}\end{array}$ & $\begin{array}{l}\text { Zwangsstörung } \\
\qquad(N=26)^{a}\end{array}$ & $\begin{array}{c}\text { Diff. } \\
\%\end{array}$ & $d F$ & $F$ & $p$ \\
\hline Totale graue Substanz ${ }^{I}$ & $723,20 \pm 76,15$ & $719,32 \pm 55,20$ & $-0,5$ & 1,46 & 0,02 & 0,90 \\
\hline Totale weiße Substanz ${ }^{1}$ & $436,35 \pm 55,50$ & $427,43 \pm 38,42$ & $-2,0$ & 1,46 & 0,48 & 0,49 \\
\hline PFC graue Substanz ${ }^{1}$ & $89,48 \pm 12,01$ & $87,06 \pm 14,93$ & $-2,7$ & 1,46 & 0,29 & 0,59 \\
\hline PFC weiße Substanz ${ }^{1}$ & $45,60 \pm 7,82$ & $43,07 \pm 9,01$ & $-5,6$ & 1,46 & 1,65 & 0,21 \\
\hline OFC links grau ${ }^{2}$ & $8224,6 \pm 2174,5$ & $7941,5 \pm 2081,2$ & $-3,4$ & 1,46 & 0,19 & 0,67 \\
\hline OFC links weiß ${ }^{2}$ & $2553,0 \pm 799,1$ & $2670,0 \pm 1070,8$ & 4,6 & 1,46 & 0,14 & 0,71 \\
\hline OFC links total ${ }^{2}$ & $10777,5 \pm 2646,9$ & $10611,6 \pm 2911,6$ & $-1,5$ & 1,46 & 0,05 & 0,83 \\
\hline OFC rechts grau ${ }^{2}$ & $9119,8 \pm 1618,9$ & $9107,5 \pm 2026,0$ & $-0,1$ & 1,46 & 0,01 & 0,94 \\
\hline OFC rechts weiß ${ }^{2}$ & $2987,2 \pm 1029,4$ & $2845,2 \pm 952,1$ & $-4,8$ & 1,46 & 0,46 & 0,50 \\
\hline OFC rechts total ${ }^{2}$ & $12107,0 \pm 2343,9$ & $11952,7 \pm 2787,6$ & $-1,3$ & 1,46 & 0,05 & 0,83 \\
\hline OFC grau $^{2}$ & $17344,4 \pm 3292,2$ & $17049,0 \pm 3154,4$ & $-1,7$ & 1,46 & 0,06 & 0,80 \\
\hline OFC weiß ${ }^{2}$ & $5540,2 \pm 1581,9$ & $5515,3 \pm 1794,8$ & $-0,4$ & 1,46 & 0,06 & 0,81 \\
\hline OFC total $^{2}$ & $22884,5 \pm 4333,3$ & $22564,3 \pm 4619,0$ & $-1,4$ & 1,46 & 0,08 & 0,78 \\
\hline
\end{tabular}




\begin{tabular}{|c|c|c|c|c|c|c|}
\hline & $\begin{array}{l}\text { Kontrollen } \\
(N=26)^{a}\end{array}$ & $\begin{array}{l}\text { Zwangsstörung } \\
\qquad(N=26)^{a}\end{array}$ & $\begin{array}{c}\text { Diff. } \\
\%\end{array}$ & $d F$ & $F$ & $p$ \\
\hline $\begin{array}{l}\text { OFC links total / } \\
\text { (TGS+TWS) }\end{array}$ & $0,0092 \pm 0,0018$ & $0,0092 \pm 0,0021$ & $-0,7$ & 1,46 & 0,04 & 0,85 \\
\hline OFC rechts grau / TGS & $0,0126 \pm 0,0016$ & $0,0126 \pm 0,0025$ & 0,5 & 1,46 & 0,01 & 0,95 \\
\hline OFC rechts weiß / TWS & $0,0068 \pm 0,00023$ & $0,0066 \pm 0,002$ & $-3,3$ & 1,46 & 0,35 & 0,56 \\
\hline $\begin{array}{c}\text { OFC rechts total / } \\
\text { (TGS+TWS) }\end{array}$ & $0,0104 \pm 0,0016$ & $0,0104 \pm 0,0021$ & $-0,2$ & 1,46 & 0,02 & 0,89 \\
\hline OFC grau / TGS & $0,239 \pm 0,003$ & $0,0236 \pm 0,0033$ & $-1,1$ & 1,46 & 0,13 & 0,72 \\
\hline $\begin{array}{l}\text { OFC links total/ } \\
\text { PFC-(GS+WS) }\end{array}$ & $0,1664 \pm 0,0317$ & $0,1702 \pm 0,0312$ & 2,3 & 1,46 & 0,22 & 0,64 \\
\hline $\begin{array}{l}\text { OFC rechts total / } \\
\text { PFC-(GS +WS) }\end{array}$ & $0,1724 \pm 0,0260$ & $0,1767 \pm 0,0339$ & 2,5 & 1,46 & 0,14 & 0,71 \\
\hline Asymm.-Koeff. Grau & $0,142 \pm 0,286$ & $0,087 \pm 0,322$ & - & 1,46 & 0,50 & 0,49 \\
\hline Asymm.-Koeff. Weiß & $0,120 \pm 0,229$ & $0,146 \pm 0,312$ & - & 1,46 & 0,12 & 0,73 \\
\hline Asymm.-Koeff. Total & $0,126 \pm 0,201$ & $0,129 \pm 0,297$ & - & 1,46 & 0,00 & 0,95 \\
\hline
\end{tabular}

Bezogen auf alle Zwangserkrankte aus der Gesamtstichprobe ergaben sich signifikante, negative Korrelationen zwischen der Krankheitsdauer und dem orbitofrontalen Volumen relativ zum präfrontalen Volumen (rechts: Graue Substanz: p.c. $=-0,53, p=0,009$, total: $p . c .=-0,45, p=0,033$; gesamte graue Substanz: p.c. $=-0,44, p=0,034)$ sowie eine signifikant positive Korrelation zwischen der Krankheitsdauer und dem Gesamthirnvolumen der grauen Substanz (p.c. $=0,42, p=0,042)$.

Weiterhin ergaben sich signifikant positive Korrelationen zwischen dem Erkrankungsalter und dem orbitofrontalen Volumen im Verhältnis zum präfrontalen Volumen (rechts: Graue Substanz: p.c. $=0,57, p=0.004$, total: p.c. $=0,51, p=$ 0,013; gesamt: Graue Substanz: p.c. $=0,46, p=0,027$ ). Bezüglich der Asymmetrie konnten keine signifikanten Veränderungen gezeigt werden, siehe Tabelle 21. 
Tabelle 21: Partielle Korrelation Zwangserkrankte für Krankheitsdauer und Alter bei Diagnosestellung

\begin{tabular}{|c|c|c|c|}
\hline & & Krankheitsdauer & $\begin{array}{c}\text { Alter bei } \\
\text { Diagnosestellung }\end{array}$ \\
\hline \multirow[t]{3}{*}{ Totale graue Substanz $^{1}$} & p.c. & 0,42 & $-0,39$ \\
\hline & $p$ & 0,042 & 0,059 \\
\hline & $d f$ & 22 & 22 \\
\hline \multirow[t]{3}{*}{ Totale weiße Substanz $^{1}$} & p.c. & 0,33 & $-0,33$ \\
\hline & $p$ & 0,11 & 0,12 \\
\hline & $d f$ & 22 & 22 \\
\hline \multirow[t]{3}{*}{ PFC graue Substanz ${ }^{1}$} & p.c. & 0,40 & $-0,37$ \\
\hline & $p$ & 0,057 & 0,081 \\
\hline & $d f$ & 21 & 21 \\
\hline \multirow[t]{3}{*}{ PFC weiße Substanz $^{1}$} & p.c. & 0,35 & $-0,34$ \\
\hline & $p$ & 0,10 & 0,11 \\
\hline & $d f$ & 21 & 21 \\
\hline \multirow[t]{3}{*}{ OFC links total ${ }^{2}$} & p.c. & 0,30 & $-0,29$ \\
\hline & $p$ & 0,16 & 0,17 \\
\hline & $d f$ & 22 & 22 \\
\hline \multirow[t]{3}{*}{ OFC rechts total ${ }^{2}$} & p.c. & $-0,03$ & 0,11 \\
\hline & $p$ & 0,90 & 0,62 \\
\hline & $d f$ & 22 & 22 \\
\hline \multirow{3}{*}{$\begin{array}{c}\text { OFC rechts grau/ } \\
\text { PFC-GS }\end{array}$} & p.c. & $-0,53$ & 0,57 \\
\hline & $p$ & 0,009 & 0,004 \\
\hline & $d f$ & 21 & 21 \\
\hline \multirow[t]{3}{*}{ OFC rechts total/ PFC } & p.c. & $-0,45$ & 0,51 \\
\hline & $p$ & 0,033 & 0,013 \\
\hline & $d f$ & 21 & 21 \\
\hline \multirow[t]{3}{*}{ OFC grau / PFC-GS } & p.c. & $-0,44$ & 0,46 \\
\hline & $p$ & 0,034 & 0,027 \\
\hline & $d f$ & 21 & 21 \\
\hline \multirow[t]{3}{*}{ Asymm.-Koeff. Grau } & p.c. & $-0,21$ & 0,27 \\
\hline & $p$ & 0,32 & 0,20 \\
\hline & $d f$ & 22 & 22 \\
\hline \multirow[t]{3}{*}{ Asymm.-Koeff. Weiß } & p.c. & $-0,31$ & 0,36 \\
\hline & $p$ & 0,14 & 0,08 \\
\hline & $d f$ & 22 & 22 \\
\hline \multirow[t]{3}{*}{ Asymm.-Koeff. Total } & p.c. & $-0,30$ & 0,35 \\
\hline & $p$ & 0,16 & 0,10 \\
\hline & $d f$ & 22 & 22 \\
\hline
\end{tabular}

Partielle Korrelation angepasst für Alter, Ausbildung und Geschlecht ; Legende: ${ }^{1}$ : in $\mathrm{cm}^{3}$; ${ }^{2}$ : in $\mathrm{mm}^{3}$; GS: Graue Substanz; WS: Weiße Substanz; Asymm. -Koeff.: Asymmetrie - Koeffizient $=\left(2^{*}(r-I) /(r+l)\right)$. 


\subsubsection{Diagnose - übergreifende Analysen}

Im Diagnose - übergreifenden Vergleich, also über alle drei Diagnosegruppen hinweg adjustiert für Alter-, Geschlechts- und Bildungsunterschiede, ergab sich für das gesamte rechte orbitofrontale Volumen relativ zum gesamten rechten präfrontalen Volumen ein signifikanter Diagnoseeffekt (OFC rechts weiße Substanz: $p=0,032$, OFC rechts total: $p=0,041$ ), dabei war das Volumen bei den bipolaren Patienten am kleinsten und bei den Zwangserkrankten am größten. Für alle anderen Zielvolumina konnte kein relevanter Diagnoseeffekt nachgewiesen werden, siehe Tabelle 22.

Im Anschluss an diese erste Diagnose - übergreifende Analyse wurden im Anschluss jeweils noch Subgruppen- Analysen zwischen einer Gruppe erkrankter Patienten und einer Kontrollgruppe mit, im Gegensatz zu den vorhergehenden Analysen, Bonferroni Korrektur durchgeführt.

In dieser Bonferroni- korrigierten Subanalyse der bipolaren Patienten zeigte sich kein signifikanter Diagnoseeffekt mehr, sondern lediglich ein Trend für eine Reduktion des gesamten weißen rechten OFC- Volumens relativ zur weißen Substanz des präfrontalen Volumens mit 0,098 sowie ein Trend zur Reduktion des gesamten rechten orbitofrontalen Volumens relativ zum gesamten präfrontalen Volumen mit 0,10.

Für alle anderen Volumina ließen sich weder Trends noch signifikante Diagnoseeffekte zeigen, siehe ebenfalls Tabelle 22.

Tabelle 22: Diagnose - übergreifende Analyse (adjustiert für Alter, Bildung und Geschlecht)

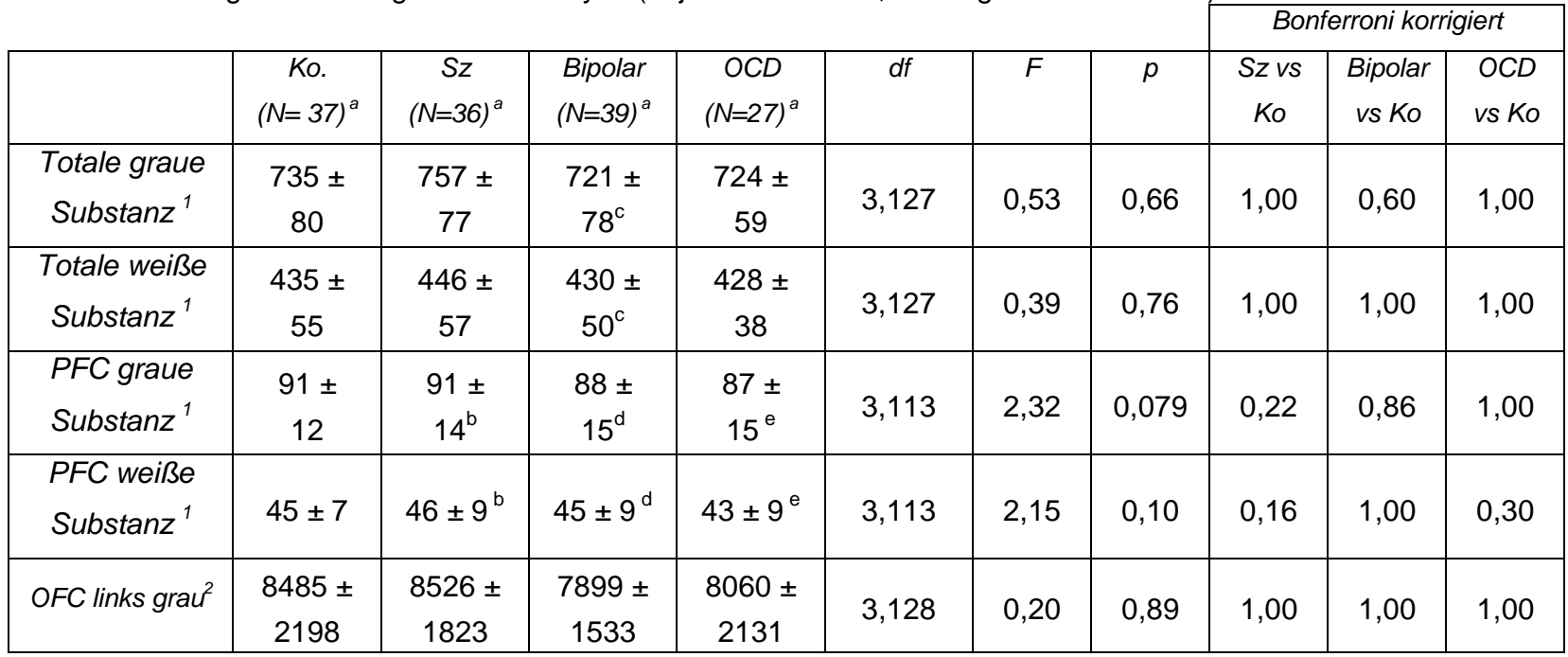




\begin{tabular}{|c|c|c|c|c|c|c|c|c|c|c|}
\hline & & & & & & & & Bon & erroni ko & giert \\
\hline & $\begin{array}{c}K o . \\
(N=37)^{a}\end{array}$ & $\begin{array}{c}S z \\
(N=36)^{a}\end{array}$ & $\begin{array}{c}\text { Bipolar } \\
(N=39)^{a}\end{array}$ & $\begin{array}{c}O C D \\
(N=27)^{a}\end{array}$ & $d f$ & $F$ & $p$ & $\begin{array}{c}\text { Sz vs } \\
\text { Ko }\end{array}$ & $\begin{array}{c}\text { Bipolar } \\
\text { vs Ko }\end{array}$ & $\begin{array}{l}\text { OCD } \\
\text { vs Ko }\end{array}$ \\
\hline $\begin{array}{c}\text { OFC links } \\
\text { weiß }^{2}\end{array}$ & $\begin{array}{c}2635 \pm \\
911\end{array}$ & $\begin{array}{c}2540 \pm \\
638\end{array}$ & $\begin{array}{c}2495 \pm \\
849\end{array}$ & $\begin{array}{c}2698 \pm \\
1060\end{array}$ & 3,128 & 0,60 & 0,62 & 0,69 & 1,00 & 1,00 \\
\hline $\begin{array}{c}\text { OFC links } \\
\text { total }^{2}\end{array}$ & $\begin{array}{c}11120 \pm \\
2777\end{array}$ & $\begin{array}{c}11066 \pm \\
2281\end{array}$ & $\begin{array}{c}10395 \pm \\
2030\end{array}$ & $\begin{array}{c}10757 \pm \\
2953\end{array}$ & 3,128 & 0,26 & 0,86 & 0,79 & 1,00 & 1,00 \\
\hline $\begin{array}{c}\text { OFC rechts } \\
\text { grau }^{2}\end{array}$ & $\begin{array}{c}9155 \pm \\
2199\end{array}$ & $\begin{array}{c}9198 \pm \\
2348\end{array}$ & $\begin{array}{c}8357 \pm \\
1975\end{array}$ & $\begin{array}{c}9175 \pm \\
2017\end{array}$ & 3,128 & 1,08 & 0,36 & 0,11 & 1,00 & 1,00 \\
\hline $\begin{array}{c}\text { OFC rechts } \\
\text { weiß }^{2}\end{array}$ & $\begin{array}{c}2976 \pm \\
1389\end{array}$ & $\begin{array}{c}2776 \pm \\
846\end{array}$ & $\begin{array}{c}2405 \pm \\
618\end{array}$ & $\begin{array}{c}2840 \pm \\
934\end{array}$ & 3,128 & 2,03 & 0,11 & 0,59 & 0,20 & 1,00 \\
\hline $\begin{array}{c}\text { OFC rechts } \\
\text { total }^{2}\end{array}$ & $\begin{array}{c}12130 \pm \\
3367\end{array}$ & $\begin{array}{c}11973 \pm \\
2885\end{array}$ & $\begin{array}{c}10761 \pm \\
2337\end{array}$ & $\begin{array}{c}12015 \pm \\
2752\end{array}$ & 3,128 & 1,38 & 0,25 & 0,15 & 0,79 & 1,00 \\
\hline OFC grau $^{2}$ & $\begin{array}{c}17639 \pm \\
3596\end{array}$ & $\begin{array}{c}17724 \pm \\
3253\end{array}$ & $\begin{array}{c}16256 \pm \\
2764\end{array}$ & $\begin{array}{c}17234 \pm \\
3240\end{array}$ & 3,128 & 0,78 & 0,51 & 0,11 & 1,00 & 1,00 \\
\hline OFC weiß ${ }^{2}$ & $\begin{array}{c}5611 \pm \\
2099 \\
\end{array}$ & $\begin{array}{c}5315 \pm \\
1304 \\
\end{array}$ & $\begin{array}{c}4900 \pm \\
1364 \\
\end{array}$ & $\begin{array}{c}5537 \pm \\
1764 \\
\end{array}$ & 3,128 & 1,39 & 0,25 & 0,49 & 0,50 & 1,00 \\
\hline OFC total ${ }^{2}$ & $\begin{array}{c}23250 \pm \\
5220\end{array}$ & $\begin{array}{c}23039 \pm \\
4194\end{array}$ & $\begin{array}{c}21156 \pm \\
3750\end{array}$ & $\begin{array}{c}22772 \pm \\
4656\end{array}$ & 3,128 & 1,08 & 0,36 & 0,14 & 0,97 & 1,00 \\
\hline $\begin{array}{l}\text { OFC links } \\
\text { grau / TGS }\end{array}$ & $\begin{array}{c}0,0115 \\
\pm \\
0,0023\end{array}$ & $\begin{array}{c}0,0112 \\
\pm \\
0,0021\end{array}$ & $\begin{array}{c}0,0110 \\
\pm \\
0,0021^{\mathrm{c}}\end{array}$ & $\begin{array}{c}0,0110 \\
\pm \\
0,0024\end{array}$ & 3,127 & 0,19 & 0,90 & 1,00 & 1,00 & 1,00 \\
\hline $\begin{array}{c}\text { OFC links } \\
\text { weiß / TWS }\end{array}$ & $\begin{array}{c}0,0060 \\
\pm \\
0,0018\end{array}$ & $\begin{array}{c}0,0057 \pm \\
0,0057\end{array}$ & $\begin{array}{c}0,0058 \\
\pm \\
0,0017^{\mathrm{c}}\end{array}$ & $\begin{array}{c}0,0062 \\
\pm \\
0,0021\end{array}$ & 3,127 & 0,69 & 0,56 & 0,99 & 1,00 & 1,00 \\
\hline $\begin{array}{c}\text { OFC links } \\
\text { total / } \\
\text { (TGS+TWS) }\end{array}$ & $\begin{array}{c}0,0094 \\
\pm \\
0,0019\end{array}$ & $\begin{array}{c}0,0092 \\
\pm \\
0,0016\end{array}$ & $\begin{array}{c}0,0090 \\
\pm \\
0,0016^{\mathrm{c}}\end{array}$ & $\begin{array}{c}0,0092 \\
\pm \\
0,0021\end{array}$ & 3,127 & 0,25 & 0,86 & 1,00 & 1,00 & 1,00 \\
\hline $\begin{array}{l}\text { OFC right } \\
\text { grau / TGS }\end{array}$ & $\begin{array}{c}0,0124 \\
\pm \\
0,0023\end{array}$ & $\begin{array}{c}0,0121 \\
\pm \\
0,0012\end{array}$ & $\begin{array}{c}0,0115 \\
\pm \\
0,0022^{\mathrm{c}}\end{array}$ & $\begin{array}{c}0,0126 \\
\pm \\
0,0024\end{array}$ & 3,127 & 1,74 & 0,16 & 0,090 & 0,59 & 1,00 \\
\hline $\begin{array}{l}\text { OFC rechts } \\
\text { weiß / TWS }\end{array}$ & $\begin{array}{c}0,0068 \\
\pm \\
0,0028\end{array}$ & $\begin{array}{c}0,0062 \\
\pm \\
0,0016\end{array}$ & $\begin{array}{c}0,0056 \\
\pm \\
0,0013^{\mathrm{c}}\end{array}$ & $\begin{array}{c}0,0066 \\
\pm \\
0,0019\end{array}$ & 3,127 & 2,36 & 0,074 & 0,59 & 0,17 & 1,00 \\
\hline $\begin{array}{c}\text { OFC rechts } \\
\text { total/ } \\
\text { (TGS+TWS) }\end{array}$ & $\begin{array}{c}0,0103 \\
\pm \\
0,0023\end{array}$ & $\begin{array}{c}0,0099 \\
\pm \\
0,0021\end{array}$ & $\begin{array}{c}0,0093 \\
\pm \\
0,0017^{c}\end{array}$ & $\begin{array}{c}0,0104 \\
\pm \\
0,0021\end{array}$ & 3,127 & 2,20 & 0,09 & 0,12 & 0,32 & 1,00 \\
\hline $\begin{array}{c}\text { OFC grau / } \\
\text { TGS }\end{array}$ & $\begin{array}{c}0,0239 \\
\pm \\
0,0032\end{array}$ & $\begin{array}{c}0,0234 \\
\pm \\
0,0034\end{array}$ & $\begin{array}{c}0,0225 \\
\pm \\
0,0033^{\mathrm{c}}\end{array}$ & $\begin{array}{c}0,0237 \\
\pm \\
0,0032\end{array}$ & 3,127 & 1,42 & 0,24 & 0,054 & 0,53 & 1,00 \\
\hline $\begin{array}{c}\text { OFC weiß / } \\
\text { TWS }\end{array}$ & $\begin{array}{c}0,0128 \\
\pm \\
0,0041\end{array}$ & $\begin{array}{c}0,0119 \\
\pm \\
0,0023\end{array}$ & $\begin{array}{c}0,0113 \\
\pm \\
0,0027^{c}\end{array}$ & $\begin{array}{c}0,0128 \\
\pm \\
0,0034\end{array}$ & 3,127 & 1,71 & 0,17 & 0,53 & 0,44 & 1,00 \\
\hline $\begin{array}{c}\text { OFC total / } \\
\text { (TGS+TWS) }\end{array}$ & $\begin{array}{c}0,0198 \\
\pm \\
0,0032\end{array}$ & $\begin{array}{c}0,0191 \\
\pm \\
0,0027\end{array}$ & $\begin{array}{c}0,0183 \\
\pm \\
0,0026^{c}\end{array}$ & $\begin{array}{c}0,0196 \\
\pm \\
0,003\end{array}$ & 3,127 & 1,85 & 0,14 & 0,090 & 0,41 & 1,00 \\
\hline
\end{tabular}




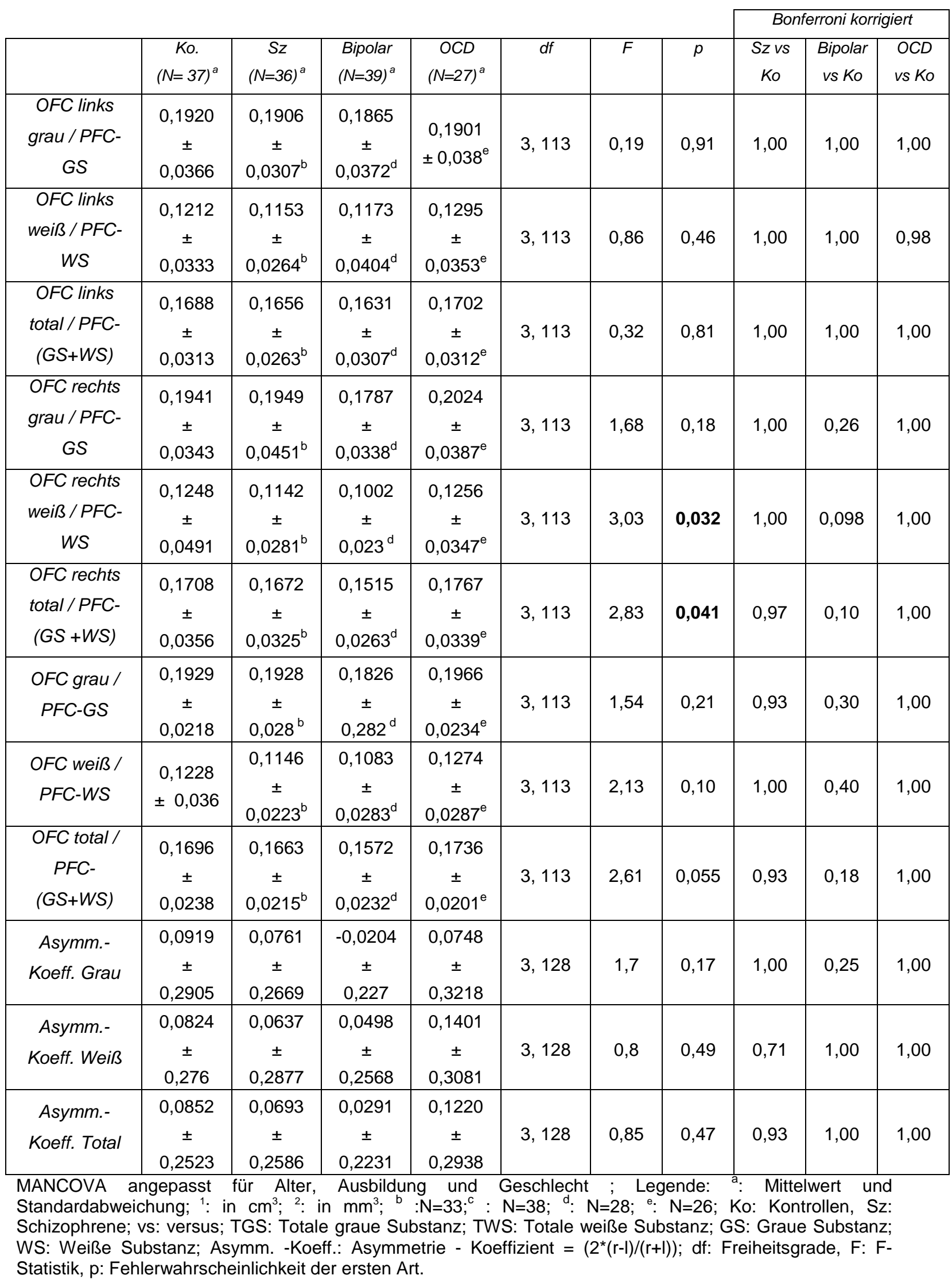




\section{Diskussion}

Zentraler Gegenstand dieser Arbeit war es, mittels manueller Ausmessung von kraniellen MRT- Bildern zu untersuchen, ob volumetrisch messbare Unterschiede des orbitofrontalen Kortex bei den drei Krankheitsgruppen Schizophrenie, bipolare Störung und Zwangserkrankung im Vergleich zu gesunden Kontrollpersonen bestehen. Hierbei wurden auch relative Volumenveränderungen in Bezug auf Gesamthirnvolumen und Präfrontalkortex berücksichtigt. Darüber hinaus bestand das Ziel, den Stellenwert etwaiger Volumendifferenzen des OFC Diagnose übergreifend auszuwerten und so eine mögliche Diagnosespezifität aufzuzeigen. Ebenso diente diese Arbeit zur Identifikation von Einflussfaktoren auf die Größe des orbitofrontalen Kortex.

Die exakte volumetrische Untersuchung des orbitofrontalen Kortex stellte sich insofern als schwierig dar, als dass die Markierung der Landmarken zur Begrenzung des Areals nicht einheitlich definiert sind. So waren in der Literatur verschiedene Vorschläge zur Begrenzung des Areals zu finden. In dieser Studie wurde die Grenzfindung angelehnt an eine Arbeit von Crespo-Facorro et al. (1999), die in ihrer Studie die Reliabilität der Methode unter Verwendung einer ähnlichen Software wie in der hier vorgelegten Arbeit zeigen konnten. Die Reliabilität der Grenzfestlegung konnte in der hier vorgelegten Arbeit anhand von jeweils 10 zufällig gewählten Fällen, welche verblindet doppelt markiert wurden, mit der Berechnung von einer hohen Retest- und Interraterreliabilität mit ICC Werten von über $>0,9$ bestätigt werden.

Bei der eingehenden Untersuchung konnten einige intervenierende Variablen auf die Volumina des orbitofrontalen Kortex gefunden werden. Sowohl das Gesamthirnvolumen als auch das orbitofrontale Volumen stellte sich bei den weiblichen Studienteilnehmern signifikant kleiner als bei den männlichen dar. Dieser Geschlechtereffekt wurde erstmals in vivo durch Gur et al. (1991) mittels volumetrischer Auswertung von kraniellen MRT- Bilder nachgewiesen. Sie zeigten, dass das männliche Gehirn im Durchschnitt um $91 \mathrm{ml}$ und das Ventrikelsystem um $20 \mathrm{ml}$ größer ist als das der weiblichen Studienteilnehmer. 
Das Alter der Studienteilnehmer korrelierte in der hier vorliegenden Studie negativ mit den Zielvolumina, was am ehesten mit einer altersbedingten Regression des Hirnvolumens zu erklären ist. Gur et al. (1991) haben ebenfalls für beide Geschlechter zeigen können, dass es eine negative Korrelation zwischen Lebensalter und Gehirnvolumen sowie eine positive Korrelation zum Ventrikelvolumen gibt. Interessanterweise konnten sie auch zeigen, dass es in weiblichen Gehirnen zu einer symmetrischen Atrophie kommt und in männlichen Gehirnen zu einer vorwiegenden Atrophie der linken Hemisphäre.

Die Dauer der Ausbildung gemessen in Schuljahren korrelierte in dieser Arbeit zum Teil positiv mit dem Volumen. Für den Faktor Händigkeit konnten keine relevanten Korrelationen mit den Zielgrößen gefunden werden.

Aufgrund dieser gefundenen Einflussfaktoren Alter, Geschlecht und Bildung wurden bei einigen der anschließend durchgeführten Analysen der Subgruppen in soziodemografischer Hinsicht gematchte Samples verwendet. Bei der Diagnose übergreifenden Analyse erfolgte eine Adjustierung für ebendiese Faktoren.

In dem soziodemografisch ausgeglichenen Sample für die schizophrenen Patienten konnten im Vergleich zu den gesunden Studienteilnehmern lediglich nicht signifikante Volumenreduktionen des orbitofrontalen Kortex gezeigt werden. Es ergaben sich eine 4,9\%ige Volumenreduktion der grauen Substanz des orbitofrontalen Kortex relativ zum gesamten grauen Kortex sowie eine 3,7\%ige Reduktion des absoluten rechten orbitofrontalen Volumen. Diese Volumendifferenzen waren mit $p=0,093$ bzw. $p=0,20$ allerdings nur als Trends nachzuweisen.

Die bisher in der Literatur vorliegenden Daten zu dieser Fragestellung sind inkonsistent. So berichten einige Autoren über Volumenzunahmen, andere über Volumenabnahmen, wieder andere konnten keine relevanten Veränderungen zeigen (Nakamura et al. 2008). Die Inkonsistenz der Ergebnisse wird am ehesten bedingt durch die hohe interindividuelle anatomische Variabilität der Zielregion und der daraus resultierenden Schwierigkeiten einer genauen Grenzfestlegung. Dies macht die manuelle Markierung schwierig. 
In dieser Studie konnte keine signifikante Volumenabnahme des orbitofrontalen Kortex bei den an Schizophrenie erkrankten Probanden gezeigt werden. Dennoch fiel in der Subgruppenanalyse auf, dass es signifikante Volumenverminderungen des orbitofrontalen Kortex bei den Patienten mit positiver Familienanamnese, also bei Vorliegen einer Schizophrenie bei Angehörigen ersten oder zweiten Grades, im Vergleich zu den gesunden Kontrollpersonen gab. Das totale Volumen des rechten orbitofrontalen Kortex zeigte sich hier mit $p=0,018$ um 12,6\% reduziert. Dieser Effekt ließ sich auch isoliert für die graue und weiße Substanz darstellen. Für den linken orbitofrontalen Kortex konnten nur leichte Trends zur Reduktion gezeigt werden, allerdings keine signifikanten Volumenabnahmen.

Diese aufgezeigten Resultate deuten auf die Relevanz familiärer bzw. genetischer Einflüsse in Bezug auf die Reduktion des orbitofrontalen Volumens bei schizophren Erkrankten hin.

Im Hinblick auf eine genetische Beziehung zwischen Struktur beziehungsweise Volumen des orbitofrontalen Kortex und dem Krankheitsbild der Schizophrenie ist die Betrachtung von Zwillingsstudien von besonderer Bedeutung. Da Geschwister und zweieiige Zwillinge im Durchschnitt 50\% ihres Erbmaterials gemeinsam haben, eineiige Zwillinge jedoch 100\%, können bei der Betrachtung beider Gruppen weitere Hinweise für das Vorhandensein einer genetischen Komponente gewonnen werden. Eine generelle Einschränkung bei der Interpretation von Zwillingsstudien besteht allerdings darin, dass gemeinsame äußere Einflüsse wie intrauterine Noxen oder ähnliches nicht mit Sicherheit als Ursache der gemessenen zerebralen Veränderungen auszuschließen sind.

In einer Voxel- basierten Arbeit von Hulshoff Pol et al. (2006) wurden monozygote und dizygote gleichgeschlechtliche Zwillinge, jeweils diskordant für Schizophrenie, gesunden Zwillingspaaren gegenüberstellt. Bei den an Schizophrenie erkrankten Zwillingskindern zeigte sich im Vergleich zur Kontrollgruppe eine signifikant verminderte Dichte der grauen Substanz sowie eine erhöhten Dichte der weißen Substanz des linken orbitofrontalen Kortex. Bei den monozygoten gesunden KoZwillingen zeigte sich eine solche Dichteänderung gleichermaßen. Bei den gesunden dizygoten Ko- Zwillingen hingegen konnte dieser Effekt nicht gezeigt werden. Hier stellten sich die Dichteverhältnisse vergleichbar mit den gesunden Kontrollpersonen dar. 
Eine andere MR- volumetrische Zwillingsstudie von Harms et al. (2010) verglich den präfrontalen Kortex von 26 Zwillingspaaren, wiederum diskordant für Schizophrenie, mit 40 gesunden Kontrollpaaren. Die kraniellen MRT-Bilder sind jeweils manuell vermessen worden. Während bei den schizophren Erkrankten eine Volumenreduktion der grauen Substanz im mittleren und inferioren frontalen Gyrus gefunden werden konnte, war dies für die gesunden Zwillingsgeschwister lediglich für den Gyrus frontalis inferior zu finden. Harms et al. haben dieses Ergebnis als Hinweis auf einen Endophänotyp für Schizophrenie gedeutet.

Sowohl die Ergebnisse dieser Dissertation als auch die der beiden vorhergehend aufgezeigten Zwillingsstudien deuten stark auf das Vorhandensein einer genetischen Komponente bezüglich Volumenreduktionen im Präfrontalkortex bei an Schizophrenie erkrankten Patienten hin. In der Literatur sind allerdings auch widersprüchliche Daten zu finden. So konnte beispielsweise in einer größer angelegten Studie von Goldman et al. (2008) zwischen 183 gesunden Geschwistern von schizophren Erkrankten und gesunden, familiär nicht belasteten Kontrollpersonen in einer volumetrischen Studie des gesamten Gehirns für keine Struktur eine signifikante Volumendifferenz gefunden werden, was gegen das Vorhandensein eines Endophänotyps spricht. Dabei ist allerdings anzumerken, dass in jener Arbeit eine automatische Sequenzierung durchgeführt wurde. Diese ungleiche Methode kann möglicherweise Ursache der inkongruenten Ergebnissen sein.

Welche genetischen Konstellationen im Einzelnen zu kortikalen oder sogar noch spezieller zu orbitofrontalen Veränderungen bei mit Schizophrenie belasteten Familien führen könnte, ist bisher nicht klar.

Eine Voxel- basierte Studie von Gurling et al. (2006) konnte einen Zusammenhang zwischen bestimmten Polymorphismen auf dem PCM 1 (pericentriolar material 1) Gen und einer Reduktion des linken orbitofrontalen Kortex bei schizophrenen Patienten darstellen. Das PCM 1 Gen ist auf Chromosom 8p22 lokalisiert und ist beteiligt an der Integrität der Zentrosomen sowie der Regulation der Mikrotubuli. Es hat somit Einfluss auf die Struktur des Zytoskeletts. Das auf dem PCM 1 Gen kodierte Protein weist strukturelle Ähnlichkeiten zum Myosin Protein auf und es ist sehr wahrscheinlich, dass PCM 1 eine Rolle in der Entwicklung des 
Nervensystems sowie der neuronalen Aktivität spielt. In die Studie eingeschlossen wurden insgesamt 450 Patienten mit Schizophrenie. Gurling et al. konnten zeigen, dass die Patienten mit PCM 1- positiver Schizophrenie einen signifikant kleineren orbitofrontalen Kortex aufwiesen als solche mit PCM 1- negativer Schizophrenie. Letztere zeigten statt dessen ausgeprägtere Volumenreduktionen im Temporallappen und Hippokampus.

Ob Mutationen im PCM 1 Gen auch außerhalb einer Schizophrenieerkrankung einen Einfluss auf die Größe des orbitofrontalen Kortex haben, ist bisher nicht untersucht worden.

In der hier vorgelegten Arbeit wurde keine genetische Charakterisierung hinsichtlich des PCM 1 Gens vorgenommen.

Wie voranstehend aufgezeigt, gibt es also aufgrund von volumetrischen Studien an gesunden Familienmitgliedern von schizophren Erkrankten Anzeichen dafür, dass es einen „volumetrischen Endophänotyp“ für Schizophrenie geben kann. Dessen ungeachtet sind die an den schizophrenen Patienten erhobenen Daten bislang inkonsistent was volumetrische Veränderungen angeht. Möglicherweise ist der Grund darin zu sehen, dass die Mehrzahl der bisher veröffentlichen volumetrischen Studien sich bevorzugt mit chronisch schizophrenen Patienten und nicht mit Ersterkrankten beschäftigt hat. Es konnte bislang nicht eindeutig geklärt werden, ob gemessene Veränderungen im Sinne von Volumenreduktionen als krankheitsverursachend oder eher als ein Prozess bzw. Folge der Krankheit gedeutet werden müssen. Weiterhin besteht Unklarheit darüber, ob die in longitudinalen Studien gemessenen volumetrischen Effekte auf das Gehirn Ausdruck eines „toxischen“ Krankheitsverlaufs, also durch diesen selbst bedingt sind, oder als direkte Folge einer konventionellen antipsychotischen Medikation zu werten sind. Möglich wäre auch, dass genau das Gegenteil der Fall ist und atypische Antipsychotika strukturelle Hirnveränderungen aufhalten und bei hoher Medikamenten Compliance ein geringerer Progress der Volumenalterationen zu verzeichnen ist (Smieskova et al. 2009).

In einer Review von Scherk und Falkai (2006) wurden 14 Studien eingeschlossen, die sich mit dem volumetrischen Effekt einer antipsychotischen Therapie beschäftigt haben. Es wurde festgestellt, dass es unter der Therapie mit typischen 
Neuroleptika zu einer Volumenzunahme der Basalganglien sowie einer Volumenabnahme der grauen Substanz verschiedener kortikaler Regionen kommt. Diese Effekte waren bereits nach 12- wöchiger Behandlung feststellbar. Unter einer Therapie mit atypischen Neuroleptika konnten hingegen vergrößerte Volumina bestimmter kortikaler Bezirke sowie des Thalamus, hingegen keine Veränderung der Basalganglien gezeigt werden. Interessanterweise führte eine Therapieumstellung von typischen auf atypische Neuroleptika sogar wieder zu einer Normalisierung des Basalganglienvolumens.

In der hier vorliegenden Arbeit konnte für die Höhe der antipsychotischen Medikation, gemessen in Chlorpromazin- Äquivalenten, eine signifikant positive Korrelation des Volumens des gesamten linken orbitofrontalen Kortex relativ zum Gesamthirnvolumen sowie zum Präfrontalvolumen mit $p=0,036$ sowie $p=0,019$ gezeigt werden. Dies spricht klar dagegen, dass in diesem Sample die Medikation zu Volumenreduktionen in diesem Bereich geführt haben könnte.

Die schizophrenen ersterkrankten Patienten in dieser Studie haben alle lediglich atypische Neuroleptika eingenommen. Für diese Gruppe der atypischen Neuroleptika konnte im Rattenmodell gezeigt werden, dass es unter der Medikation zu einer Proliferation präfrontaler Gliazellen und letztlich zu einer kortikalen Hypertrophie im präfrontalen Kortex sowie Striatum kommt. Für typische Neuroleptika wie Haloperidol gilt dies hingegen nicht (Wang et al. 2004). Molina et al. (2007) führten diesbezüglich eine Studie an 11 mit dem atypischen Neuroleptikum Olanzapin behandelten schizophrenen Patienten und gesunden Kontrollpersonen durch und kamen zu einem anderen Ergebnis. Zu Beginn der Studie zeigten die Patienten keine signifikanten Unterschiede in Bezug auf das frontale oder parietale Volumen verglichen mit den gesunden Kontrollpersonen. Es konnten allerdings longitudinale Veränderungen im Sinne einer größeren Volumenabnahme sowohl frontal als auch parietal verglichen mit den Kontrollpersonen gemessen werden. Diese Volumenveränderungen standen dabei in keinem statistischen Zusammenhang mit individuellen Volumendeviationen zu Beginn der Studie oder mit der Besserung der klinischen Symptomatik. Um auszuschließen, dass die gemessenen Veränderungen mit einer vorhergehenden medikamentösen Therapie zusammenhängen könnten, 
wurde ein kleineres Sample nur aus den zu Beginn der Studie 7 therapie-naiven Patienten zusammengestellt. Hier konnte der gleiche Effekt gemessen werden (Molina et al. 2007). Damit zeigten auch atypisch behandelte Patienten in dieser kleinen Studie eine frontoparietale Volumenabnahme.

Interessanterweise konnten Molina et al. (2004) auch zeigen, dass es einen direkten Zusammenhang zwischen der Atrophie des orbitofrontalen Kortex sowie dem Therapieansprechen auf Olanzapin gibt. So haben wider Erwarten die Patienten mit einer ausgeprägteren Volumenreduktion des orbitfrontalen Kortex klinisch deutlicher von der Therapie profitiert.

Zwischen der Dauer der Psychose und den Zielvolumina des orbitofrontalen Kortex konnten in unserer Studie keine Zusammenhänge abgeleitet werden. Allerdings ist anzumerken, dass nur ersterkrankte Schizophrene im Kollektiv berücksichtigt worden und die Psychosedauer im Mittel nur 45,72 Wochen, also weniger als 1 Jahr, betrug. Insofern ist der Vergleich mit den bisher in der Literatur untersuchten Samples von chronisch Kranken nur bedingt möglich.

Pantelis et al. (2003) konnten in einer longitudinalen Studie Volumenreduktionen des orbitofrontalen Kortex bereits bei prodromalen Patienten mit einem späteren Übergang in eine Psychose zeigen. Dort wurden 75 Probanden untersucht, die alle als Hochrisikopatienten zur Entwicklung einer Schizophrenie galten. Es zeigte sich, dass es bereits zerebrale Volumenreduktionen im Vergleich zu Gesunden gab, bevor es zur klinischen Manifestation einer Psychose kam. Aus dem Gesamtkollektiv entwickelten nach einem Jahr 23 Personen eine Psychose. Im Vergleich zu den später nicht erkrankten Personen konnte hier retrospektiv bereits zu Beginn der Studie, also an bisher nicht therapierten und nicht manifest an Schizophrenie erkrankten Probanden, eine signifikante MR- volumetrische Verkleinerung unter anderem des rechten präfrontalen Kortex gezeigt werden (Pantelis et al. 2003).

Um noch differenzierter zwischen Ursache und Folge zerebraler volumetrischer Veränderungen im Rahmen einer Schizophrenie differenzieren zu können, werden allerdings noch weitere Studien benötigt. Die Arbeit von Pantelis et al. legt jedoch nahe, dass eine Reduktion des OFC- Volumens das Risiko für einen späteren Übergang in die Psychose deutlich erhöhen könnte. 
Die in dieser Arbeit untersuchten verschiedenen klinischen Scores der Skalen wie Clinical Global Impressions (CGI), Global Assessment Of Functioning (GAF) und die Positive And Negative Syndrome Scale (PANSS) zeigten allesamt keine signifikanten Einflüsse auf die gemessenen Volumina. Es konnte in dieser Arbeit demnach kein Zusammenhang zwischen dem generellen Schweregrad der Erkrankung, dem psychosozialen Funktionsniveau oder der klinischen Ausprägung der schizophrenen Symptomatik bei stationärer Aufnahme und dem Volumen des orbitofrontalen Kortex gezeigt werden. Der longitudinale Verlauf wurde in der hiesigen Arbeit allerdings nicht untersucht.

Zusammenfassend ist zu sagen, dass die hier vorgelegten Daten an Ersterkrankten in Übereinstimmung mit bereits bestehender Literatur stark vermuten lassen, dass es volumetrische Veränderungen des orbitofrontalen Kortex bei schizophrenen Patienten bereits früh im Verlauf der Erkrankung gibt, ggf. schon vor Ausbruch der klinischen Symptomatik und demnach auch vor Beginn einer medikamentösen Therapie.

Diese Volumenreduktionen scheinen aber, zumindest teilweise, von der familiären Belastung abhängig zu sein. Ein wesentliches und so in der Literatur noch nicht gefundenes Ergebnis dieser Dissertation ist, dass ein höherer familiärer Krankheitsload und nicht so sehr das Vorhandensein der schizophrenen Erkrankung an sich, mit einem geringeren orbitofrontalen Volumen assoziiert ist. Durch weitere Untersuchungen gesunder Angehöriger von schizophrenen Patienten mit und ohne familiäre Vorbelastung könnte weiter geklärt werden, ob es sich dabei eventuell um einen Endophänotyp handelt.

Anzumerken ist allerdings, dass die in der Literatur angegebenen Volumenreduktionen in den jeweils untersuchten Samples schizophrener Probanden teilweise für den rechten orbitofrontalen Kortex und teilweise für den linken beschrieben wurden. Es bedarf hier also noch weiterer Bemühungen, die vor allem eine Klärung der familiären genetischen Einflüsse speziell auf die rechte bzw. die linke Hemisphäre einbezieht. Crow (2008) hat bereits vor einigen Jahren postuliert, dass die zerebrale Spezialisierung des menschlichen Gehirns, welche einst mit der Entwicklung des Sprachverständnisses des Menschen einherging, 
auch zu einem erhöhten Risiko für die Entwicklung schizophrener Symptome geführt hat. Personen, denen die typische Dominanz der linken Hemisphäre im Bezug auf Sprache fehlt, haben demnach ein erhöhtes Risiko für die Entwicklung typischer Symptome wie auditorische Halluzinationen. Veränderte Lateralisierung konnte sowohl in strukturellen als auch funktionellen Untersuchungen der Gehirne von an Schizophrenie erkrankten Patienten gezeigt werden. Crow folgerte, dass die Hauptursache in einer Unterbrechung der linguistischen Spezialisierung der Hemispähren liegt (Crow 2008; Spironelli et al. 2008). In der hier vorliegenden Arbeit sind diese Effekte nicht untersucht worden.

Nach der Schizophrenie ist in dieser Arbeit als zweite Krankheitsgruppe die bipolare Störung untersucht worden. Bezüglich der euthymen bipolaren Patienten konnte verglichen mit gesunden Kontrollpersonen eine signifikante Volumenabnahme des rechten orbitofrontalen Kortex in Relation zum Gesamtkortex sowie zum Präfrontalkortex gezeigt werden. Für die absoluten Größen des OFC konnten allerdings nur Trends zur Reduktion dargestellt werden. So präsentierte sich vor allem der rechte orbitofrontale Kortex um 16,3\% ( $p=$ $0,065)$ kleiner bei den bipolaren Patienten.

Diese Ergebnisse sind ähnlich denen einer aktuellen Studie von Nery et al. (2009), in der ebenfalls eine manuelle volumetrische Ausmessung des orbitofrontalen Kortex bei bipolaren und gesunden Probanden stattfand. Dort konnten ebenfalls nur Trends bezüglich einer absoluten Volumenabnahme gezeigt werden. Die Studie schloss allerdings neben euthymen auch derzeit manische und depressive Patienten ein. Hierbei fiel auf, dass das orbitofrontale Volumen bei den zum Zeitpunkt der Bildgebung depressiven Patienten signifikant kleiner war als bei den euthymen Patienten. Mit Hilfe des Hamilton Depression Rating Scores (HDRS) konnten Nery et al. sogar Hinweise darauf finden, dass der Schweregrad der depressiven Verstimmung negativ mit dem orbitofrontalen Volumen korreliert.

Wie bereits im Vorfeld geschildert, sind in der hier vorliegenden Arbeit nur Patienten berücksichtigt worden die zum Zeitpunkt der kraniellen Bildgebung euthym waren. Wir konnten jedoch eine negative Korrelation zwischen Anzahl der depressiven Episoden und Volumen der rechten grauen orbitofrontalen Substanz 
mit rho $=-0,36$ und $p=0,043$ finden. Damit scheint sich auch in dieser Arbeit anzudeuten, dass ein schwerer Krankheitsverlauf mit einer höheren Volumenreduktion assoziiert ist. Es konnte aber kein Zusammenhang zwischen den Volumina und dem klinischen MADRS Summenscore, also dem Schweregrad der aktuellen depressiven Symptomatik, gezeigt werden. Dies entspricht jedoch den Erwartungen, da die untersuchten Patienten euthym waren.

Bezüglich einer Therapie mit dem Stimmungsstabilisator Lithium konnten wir in dieser Studie bei den bipolaren Patienten mit im Vergleich zu denen ohne Lithiumtherapie sowohl für den rechten als auch für den linken totalen orbitofrontalen Kortex eine Volumenzunahme um jeweils 5,3\% messen. Diese Differenzen liegen allerdings mit $p=0,32$ bzw. $p=0,36$ unterhalb des Signifikanzniveaus.

Lithium ist bereits seit mehr als 50 Jahren das Standard- oder ReferenzMedikament in der Rezidivprophylaxe der bipolaren Störung, dennoch ist der genaue therapeutische Wirkmechanismus auf zellulärer Ebene noch nicht hinreichend geklärt worden. Moore et al. (2000) haben in einer Studie gezeigt, dass eine dauerhafte Lithiumtherapie zu einer Zunahme der grauen Substanz führt. Für die weiße Substanz konnten keine statistisch signifikanten Veränderungen festgestellt werden. Zwischen den bipolaren Patienten die keine Lithiumtherapie erhielten und gesunden Kontrollpersonen konnten keine Volumendifferenzen gezeigt werden. Die Ergebnisse weisen demnach deutlich daraufhin, dass die gemessenen Effekte in der Tat auf die Lithiumtherapie zurückzuführen sind. Bemerkenswert ist allerdings, dass keine Beziehung zwischen Lithiumdosis oder Blutspiegel und den Änderungen der Volumina gezeigt werden konnte (Moore et al. 2000).

In der bereits erwähnten volumetrischen Studie von Nery et al. (2009) konnten für die mit Lithium therapierten Patienten ebenfalls geringfügig vergrößerte Volumina gemessen werden, allerdings lagen diese auch unterhalb des Signifikanzniveaus. Bearden et al. (2007) schlussfolgerten in ihrer Studie, dass Lithium als Stimmungsstabilisator einen neuroprotektiven Effekt auf das ZNS hat und dies der Grund sein könnte für die bisher inkongruenten Ergebnisse volumetrischer Studien bei bipolaren Patienten. Manji et al (2000) konnten in Ihrer Studie bereits vor 
einigen Jahren zeigen, dass sowohl Lithium als auch Valproinsäure zu einer deutlichen Zunahme des B-Zell-Lymphom Proteins 2 (Bcl-2) Spiegels im Frontalhirn, Hippocampus und Striatum in vivo führt. Darüber hinaus konnte eine Inhibition der Glykogen Synthase Kinase- $3 \beta$ (GSK-3 $\beta$ ) gezeigt werden (Manji et al. 2000). Bcl-2 ist Teil einer gut erforschten Proteinfamilie, die an der Regulation des apoptotischen Zelltodes beteiligt ist. Es ist im Mitochondrium lokalisiert und inhibiert die Freisetzung proapoptotischer Faktoren. GSK-3 $\beta$ stellt einen Knotenpunkt verschiedener Signalwege zur Induktion des Zelltodes dar. Mindestens über diese beiden Mechanismen scheinen sowohl Lithium als auch Valproinsäure einen anti- apoptotischen Effekt auf neuronale Zellen zu haben (Bielecka und Obuchowicz 2008; Mora et al. 2001).

Für die bipolaren Patienten konnten, unabhängig von einer Lithiumtherapie, bereits in post mortem Studien zerebrale Volumenreduktionen (Cotter et al. 2005) sowie Veränderungen auf zellulärer Ebene im Sinne einer reduzierten Dichte an Gliazellen festgestellt werden (Toro et al. 2006).

Die in dieser Studie gewonnen Ergebnisse stimmen also grundsätzlich mit denen in der Literatur beschriebenen Effekten überein. Demnach scheint es bei bipolaren Patienten zu Volumenreduktionen im orbitofrontalen Bereich zu kommen. Einen gegenteiligen Effekt hat offenbar eine Therapie mit dem, möglicherweise als generell neuroprotektiv anzusehenden, Stimmungsstabilisator Lithium.

Bezüglich der dritten Krankheitsgruppe, der Zwangserkrankung, konnten in dieser Arbeit keine statistisch signifikanten Volumenalterationen des orbitofrontalen Kortex zwischen Erkrankten und gematchten Kontrollpersonen gezeigt werden. Allerdings konnten wir in unserer Stichprobe Hinweise dafür finden, dass sich das orbitofrontale Volumen in Abhängigkeit von Beginn und Dauer der Erkrankung verändert. So konnte in einer für Alter und Bildung angepassten Analyse eine statistisch signifikante negative Korrelation mit $p=0,009$ zwischen Dauer der Zwangserkrankung und relativem grauen Volumen des rechten orbitofrontalen im Verhältnis zum präfrontalen Kortex dargestellt werden. Das heißt, je länger das Bestehen der Zwangserkrankung, desto kleiner war das relative Volumen des orbitofrontalen Kortex. Weiterhin korrelierte das Alter bei Erstdiagnosestellung 
positiv mit dem relativen rechten orbitofrontalen Volumen (grau: $p=0,004$, total: $p=0,013)$. Je früher die Patienten also erkrankten, desto deutlicher zeigte sich der Volumenverlust.

Insgesamt entsprachen diese Ergebnisse nicht den Erwartungen die aufgrund einer aktuellen Meta Analyse von Rotge et al. (2009) zu vermuten waren. In jene Analyse wurden 21 Studien einbezogen. Die Ergebnisse, auch in Bezug auf die Volumina des orbitofrontalen Kortex, waren uneinheitlich. Während einige Autoren keine messbaren Veränderungen darstellen konnten, stellten die meisten Autoren eine Volumenreduktion des orbitofrontalen Kortex sowie eine Volumenzunahme des Thalamus fest. Bei der Analyse der Daten deutete es sich für die Autoren an, dass Krankheitsdauer, Dauer der unbehandelten Symptome oder Dauer und Dosis der medikamentösen Therapie einen Einfluss auf die Volumenreduktion haben. Aufgrund der ungenügenden Datenlage konnten dazu jedoch keine genaueren Analysen gemacht werden (Rotge et al. 2009).

Neben diesen möglichen strukturellen Veränderungen des orbitofrontalen Kortex sind in der Literatur auch funktionelle Zusammenhänge zwischen der Zwangssymptomatik und dem orbitofrontalen Kortex zu finden. In einer bereits eingangs erwähnten Arbeit von Roth et al. (2007) wurden funktionelle MRTs von 12 Probanden mit Zwangsstörung mit denen gesunder Kontrollpersonen verglichen. Es fanden sich negative Korrelationen zwischen dem Schweregrad der Zwangsstörung und der Aktivität im rechten orbitofrontalen Kortex während der Impulsunterdrückung. Demnach wäre es möglich, dass die Befähigung zur Aktivierung des orbitofrontalen Kortex mit einer gesteigerten Unterdrückung von Zwangshandlungen einhergeht.

Zusammenfassend ist zu sagen, dass die volumetrischen Ergebnisse dieser Arbeit für die Zwangserkrankten nicht mit der überwiegenden Anzahl der Studien übereinstimmen. So konnten wir keine generellen Volumenreduktionen bei Zwangserkrankten feststellen, sondern lediglich einen Hinweis auf Zusammenhänge zwischen Krankheitsdauer bzw. Erkrankungsbeginn und Volumenreduktion zeigen. Da in dieser Arbeit weder Dosis noch Dauer einer medikamentösen Therapie betrachtet wurde, können auch keine Aussagen über 
einen möglichen Therapieeffekt gemacht werden. Bei der Betrachtung der zur Verfügung stehenden Literatur ist jedoch auffällig, dass viele Autoren ebenfalls Schwierigkeiten mit dem Nachweis volumetrischer Veränderungen bei Zwangserkrankten hatten. Wie Rotge et al. (2009) vermuten, liegt dies am ehesten an fehlenden Daten zum Effekt einer Medikation beziehungsweise auf an der fehlenden Datenlage bezüglich Dauer der unbehandelten Symptomatik. Es sind also diesbezüglich noch weitere Studien notwendig. Insbesondere was den Effekt einer medikamentösen Therapie betrifft.

Die Diagnose - übergreifenden Analysen galten hauptsächlich der Fragestellung, ob es eine gewisse Krankheitsspezifität für Volumenveränderungen des orbitofrontalen Kortex gibt. Eine solche Spezifität konnten anhand der Datenlage in dieser Arbeit nicht gezeigt werden. Keine der untersuchten Krankheitsgruppen, insbesondere auch nicht die schizophren Erkrankten, zeigten signifikant reduzierte Volumina des orbitofrontalen Kortex im Vergleich zu den anderen Gruppen.

Die Variabilität der orbitofrontalen Volumina innerhalb einer Krankheitsgruppe war ausgesprochen hoch. Dies mag ein Grund dafür sein, dass mögliche krankheitsspezifische Unterschiede nicht gefunden werden konnten. Der besonders interessante Befund des Einflusses der familiären Belastung auf das orbitofrontale Volumen der schizophren Erkrankten konnte leider in den anderen beiden Diagnosegruppen bipolare Störung und Zwangserkrankung nicht untersucht werden, da keine Daten über die Familienanamnese vorlagen. 


\section{$5 \quad$ Zusammenfassung}

Der orbitofrontale Kortex als ein Teil des Präfrontalkortexes gilt als multimodaler Knotenpunkt der sensorischen Integration und Modulation und ist für verschiedenste höhere assoziative Vorgänge im Primatenhirn unabdingbar. Läsionsstudien zeigten, dass Schädigungen dieses Kortex Areals zu komplexen Verhaltensstörungen führen. Dysfunktionen des orbitofrontalen Kortex stehen im Zusammenhang mit dem Auftreten bestimmter neuropsychiatrischer Erkrankungen wie Schizophrenie, Zwangsstörung, Störung des Sozialverhaltens und anderen.

In dieser Arbeit wurde ein Gesamtkollektiv von 139 Probanden, darunter Schizophrene, Zwangserkrankte und bipolare Patienten sowie gesunde Kontrollpersonen, auf Volumenunterschiede des orbitofrontale Kortex untersucht. Dabei wurde das Volumen des orbitofrontalen Kortex manuell mit Hilfe der Software MRIcron anhand von definierten Grenzen erfasst. Die Markierung erfolgte bilateral und getrennt zwischen grauer und weißer Substanz. Alle erhobenen Daten wurden sowohl isoliert als absolute Volumina als auch als relative Volumina im Verhältnis zu dem Gesamthirn- und dem Präfrontalhirnvolumen betrachtet.

Entgegen unserer Erwartung konnte für die schizophrenen Patienten keine signifikante Volumenreduktion des orbitofrontalen Kortex dargestellt werden. Interessanterweise fiel aber eine signifikante Volumenminderung rechtsseitig bei Patienten mit positiver Familienanamnese für Schizophrenie auf. Damit ergab sich in unserer Studie erstmals ein deutlicher Hinweis auf die Relevanz familiärer Belastung auf das orbitofrontale Volumen. Im Zusammenhang mit bereits bestehenden Daten aus der Literatur liegt sogar die Vermutung nahe, dass eine bestehende Volumenreduktion des orbitofrontalen Kortex als bedeutender Risikofaktor für den späteren Ausbruch der schizophrenen Erkrankung angesehen werden kann. Da solche Volumenveränderungen laut der hier erhobenen Daten wenigstens zum Teil auch auf eine familiäre, eventuell genetische Komponente zurückgeführt werden können, werden weitere Studien an gesunden Angehörigen 
von an Schizophrenie erkrankten Personen gebraucht um klären können, ob es sich bei dem hier gefundenen Phänomen um einen Endophänotyp handelt.

Für die bipolaren Patienten konnten wir im Einklang mit vergleichbaren Studien eine gering ausgeprägte, relative Volumenabnahmen des orbitofrontalen Kortex messen. Für solche Patienten, die mit Lithium therapiert wurden, konnten wir in Korrelation mit der kumulativen Tagesdosis einen Trend zur orbitofrontalen Volumenzunahme feststellen. Dies entspricht weitestgehend den bisherigen Daten aus der Literatur. Aufgrund dieses Effektes wird diskutiert, ob Lithium einen neuroprotektive und anti- apoptotische Wirkung besitzt.

Für die Gruppe der Zwangserkrankten konnte entgegen der Erwartungen keine absoluten oder relativen Volumenreduktion des orbitofrontalen Kortex im Vergleich zur gesunden Kontrollgruppe gezeigt werden. Allerdings korrelierte hier ein früher Krankheitsbeginn und eine längere Dauer der Symptomatik mit kleineren orbitofrontalen Volumina. Es bleibt weiterhin $\mathrm{zu}$ untersuchen, ob die medikamentöse Therapie der Zwangserkrankung einen Einfluss auf die Zielvolumina hat.

In der Diagnose - übergreifenden Analyse konnte keine Krankheitsspezifität für eine Veränderung der orbitofrontalen Volumina dargestellt werden. Dies könnte unter anderem schon dadurch bedingt sein, dass die Variabilität der Volumina des orbitofrontalen Kortex innerhalb der einzelnen Krankheitsgruppen sehr hoch war. 


\section{Tabellenverzeichnis}

Tabelle 1: $\quad$ Soziodemografische und klinische Daten des

Gesamtkollektivs................................. 5.17

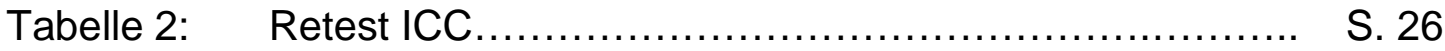

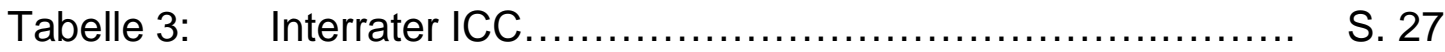

Tabelle 4: Geschlechtsabhängige Volumina aller Krankheitsgruppen

Tabelle 5: $\quad$ Bivariate Korrelation nach Pearson zwischen Alter und den abhängigen Variablen

Tabelle 6: Bivariate Korrelation nach Pearson zwischen

Bildungsdauer und den abhängigen Variablen.

Tabelle 7: $\quad$ Bivariate Korrelation nach Pearson zwischen Händigkeit und den abhängigen Variablen

S. 31

Tabelle 8: Soziodemographische Daten gematchtes Sample Schizophrenie.

Tabelle 9: Volumina schizophrene Patienten und Kontrollpersonen S. 32

Tabelle 10: Volumina schizophrene Patienten in Abhängigkeit der Familienanamnese

Tabelle 11: Partielle Korrelation schizophrene Patienten und verschiedene Faktoren

Tabelle 12: Soziodemografische Daten gematchtes Sample Bipolare

Tabelle 13: Volumina Bipolare und Kontrollpersonen S. 38

Tabelle 14: Volumina Bipolare in Abhängigkeit von

Lithiumeinnahme

Tabelle 15: Volumina Bipolare in Abhängigkeit von anderen Stimmungsaufheller als Lithium

Tabelle 16: Volumina Bipolare in Abhängigkeit von Psychotischen Symptomen

Tabelle 17: Volumina Bipolare in Abhängigkeit von

Neuroleptikaeinnahme.

Tabelle 18: Partielle Korrelation Bipolare für verschiedene Faktoren 
Tabelle 19: Soziodemographische Daten gematchtes Sample

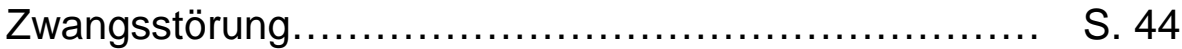

Tabelle 20: Volumina Zwangserkrankte und Kontrollpersonen........ S. 44

Tabelle 21: Partielle Korrelation Zwangserkrankte für verschiedene Faktoren .......................................... 46

Tabelle 22: Diagnose - übergreifende Analyse(adjustiert für Alter,

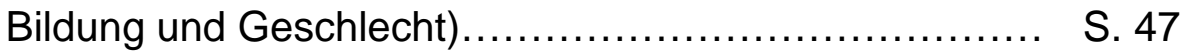




\section{Abbildungsverzeichnis}

Abbildung 1: $\quad$ Lage des orbitofrontalen Kortex (Kringelbach und Rolls 2004, S. 343)............................. S. 1

Abbildung 2: $\quad$ Zytoarchitektonische Karte der lateralen und orbitalen Oberfläche des Menschen (A) und des MakakeAffens (B) (Petrides und Pandya 2002, S.294)........ S. 2

Abbildung 3: Schema der funktionellen Konnektivität des orbitofrontalen Kortex (Kringelbach 2005, S. 700)...... S. 7

Abbildung 4: Funktionelles Modell des orbitofrontalen Kortex (Kringelbach 2005, S.700)...................... S. 8

Abbildung 5: $\quad$ Markierung der grauen Substanz OFC links............ S. 22

Abbildung 6: Markierung der grauen Substanz OFC links............ S. 22

Abbildung 7: Totales rechtes OFC Volumen bei Kontrollpersonen sowie schizophren Erkrankten mit negativer und positiver Familienanamnese

Abbildung 8: Relatives OFC Volumen in Prozent des gesamten Hirnvolumens bei Kontrollpersonen sowie schizophren Erkrankten mit negativer und positiver Familienanamnese 


\section{$8 \quad$ Literaturverzeichnis}

Altshuler L, Bookheimer S, Proenza MA, Townsend J, Sabb F, Firestine A, Bartzokis G, Mintz J, Mazziotta J,Cohen MS (2005): "Increased amygdala activation during mania: a functional magnetic resonance imaging study." Am J Psychiatry 162(6): 1211-1213.

Altshuler L, Bookheimer S, Townsend J, Proenza MA, Sabb F, Mintz J, Cohen MS 2008): "Regional brain changes in bipolar I depression: a functional magnetic resonance imaging study." Bipolar Disord 10(6): 708-717.

Anderson SW, Bechara A, Damasio H, Tranel D,Damasio AR (1999): "Impairment of social and moral behavior related to early damage in human prefrontal cortex." Nat Neurosci 2(11): 1032-1037.

Aouizerate B, Guehl D, Cuny E, Rougier A, Bioulac B, Tignol J,Burbaud P (2004): "Pathophysiology of obsessive-compulsive disorder: a necessary link between phenomenology, neuropsychology, imagery and physiology." Prog Neurobiol 72(3): 195-221.

Baare WF, Hulshoff Pol HE, Hijman R, Mali WP, Viergever MA,Kahn RS (1999): "Volumetric analysis of frontal lobe regions in schizophrenia: relation to cognitive function and symptomatology." Biol Psychiatry 455(12): 1597-1605.

Bachevalier J,Loveland KA (2006): "The orbitofrontal-amygdala circuit and selfregulation of social-emotional behavior in autism." Neurosci Biobehav Rev 30(1): 97-117.

Bagary MS, Symms MR, Barker GJ, Mutsatsa SH, Joyce EM,Ron MA (2003): "Gray and white matter brain abnormalities in first-episode schizophrenia inferred from magnetization transfer imaging." Arch Gen Psychiatry $\underline{60}(8)$ : 779-788. 
Bagary MS, Foong J, Maier M, duBoulay G, Barker GJ, Miller DH,Ron MA (2002):

"A magnetization transfer analysis of the thalamus in schizophrenia." J Neuropsychiatry Clin Neurosci 14(4): 443-448.

Barbas H,Pandya DN (1989): "Architecture and intrinsic connections of the prefrontal cortex in the rhesus monkey." J Comp Neurol 286(3): 353-375.

Barbas H,Blatt GJ (1995): "Topographically specific hippocampal projections target functionally distinct prefrontal areas in the rhesus monkey." Hippocampus $\underline{5}(6)$ : 511-533.

Bearden CE, Thompson PM, Dalwani M, Hayashi KM, Lee AD, Nicoletti M, Trakhtenbroit M, Glahn DC, Brambilla P, Sassi RB, et al. (2007): "Greater cortical gray matter density in lithium-treated patients with bipolar disorder." Biol Psychiatry $\underline{62(1): ~ 7-16 . ~}$

Bielecka AM, Obuchowicz E (2008): "Antiapoptotic action of lithium and valproate." Pharmacol Rep $\underline{60}(6)$ : 771-782.

Bonnici HM, William T, Moorhead J, Stanfield AC, Harris JM, Owens DG, Johnstone EC, Lawrie SM (2007): "Pre-frontal lobe gyrification index in schizophrenia, mental retardation and comorbid groups: an automated study." Neuroimage 35(2): 648-654.

Brodmann K (1909): "Vergleichende Lokalisationslehre der Grosshirnrinde in ihren Prinzipien dargestellt auf Grund des Zellenbaues."

Buchanan RW, Vladar K, Barta PE, Pearlson GD (1998): "Structural evaluation of the prefrontal cortex in schizophrenia." Am J Psychiatry 155(8): 1049-1055.

Carlsson ML (2001): "On the role of prefrontal cortex glutamate for the antithetical phenomenology of obsessive compulsive disorder and attention deficit hyperactivity disorder." Prog Neuropsychopharmacol Biol Psychiatry 25(1): 5-26. 
Carmichael ST, Price JL (1995a): "Sensory and premotor connections of the orbital and medial prefrontal cortex of macaque monkeys." J Comp Neurol 363(4): 642-664.

Carmichael ST, Price JL (1995b): "Limbic connections of the orbital and medial prefrontal cortex in macaque monkeys." J Comp Neurol 363(4): 615-641.

Carmichael ST, Price JL (1996): "Connectional networks within the orbital and medial prefrontal cortex of macaque monkeys." J Comp Neurol 371(2): 179207.

Carmichael ST, Clugnet MC, Price JL (1994): "Central olfactory connections in the macaque monkey." J Comp Neurol 346(3): 403-434.

Cavada C, Company T, Tejedor J, Cruz-Rizzolo RJ, Reinoso-Suarez F (2000): "The anatomical connections of the macaque monkey orbitofrontal cortex. A review." Cereb Cortex 10(3): 220-242.

Chemerinski E, Nopoulos PC, Crespo-Facorro B, Andreasen NC, Magnotta V (2002): "Morphology of the ventral frontal cortex in schizophrenia: relationship with social dysfunction." Biol Psychiatry 52(1): 1-8.

Chiavaras MM, Petrides M (2000): "Orbitofrontal sulci of the human and macaque monkey brain." J Comp Neurol 422(1): 35-54.

Chiavaras MM, LeGoualher G, Evans A, Petrides M (2001): "Three-dimensional probabilistic atlas of the human orbitofrontal sulci in standardized stereotaxic space." Neuroimage 13(3): 479-496.

Choi JS, Kang DH, Kim JJ, Ha TH, Lee JM, Youn T, Kim IY, Kim SI, Kwon JS (2004): "Left anterior subregion of orbitofrontal cortex volume reduction and impaired organizational strategies in obsessive-compulsive disorder." J Psychiatr Res 38(2): 193-199.

CIPS: "Internationale Skalen für Psychiatrie." Beltz Test, Weinheim 1986. 
Convit A, Wolf OT, de Leon MJ, Patalinjug M, Kandil E, Caraos C, Scherer A, Saint Louis LA, Cancro R (2001): "Volumetric analysis of the pre-frontal regions: findings in aging and schizophrenia." Psychiatry Res 107(2): 61-73.

Cotter D, Hudson L, Landau S (2005): "Evidence for orbitofrontal pathology in bipolar disorder and major depression, but not in schizophrenia." Bipolar Disord $\underline{7}(4)$ : 358-369.

Crespo-Facorro B, Kim JJ, Andreasen NC, O'Leary DS, Wiser AK, Bailey JM, Harris G, Magnotta VA (1999): "Human frontal cortex: an MRI-based parcellation method." Neuroimage 10(5): 500-519.

Critchley HD, Rolls ET (1996): "Hunger and satiety modify the responses of olfactory and visual neurons in the primate orbitofrontal cortex." J Neurophysiol 75(4): 1673-1686.

Crow TJ (2008): "The 'big bang' theory of the origin of psychosis and the faculty of language." Schizophr Res 102(1-3): 31-52.

Dawson G, Meltzoff AN, Osterling J, Rinaldi J (1998): "Neuropsychological correlates of early symptoms of autism." Child Dev $\underline{69}$ (5): 1276-1285.

Elliott R, Frith CD, Dolan RJ (1997): "Differential neural response to positive and negative feedback in planning and guessing tasks." Neuropsychologia 35(10): 1395-1404.

Elliott R, Ogilvie A, Rubinsztein JS, Calderon G, Dolan RJ, Sahakian BJ (2004): "Abnormal ventral frontal response during performance of an affective go/no go task in patients with mania." Biol Psychiatry $\underline{55}(12):$ 1163-1170.

Folstein MF, Folstein SE, McHugh PR (1975): "'"Mini-mental state". A practical method for grading the cognitive state of patients for the clinician." J Psychiatr Res 12(3): 189-198.

Förstl H: "Frontalhirn - Funktionen und Erkrankungen". Springer Medizin Verlag, Heidelberg 2005. 
Frey S, Petrides M (2000): "Orbitofrontal cortex: A key prefrontal region for encoding information." Proc Natl Acad Sci U S A 97(15): 8723-8727.

Frey S, Petrides M (2003): "Greater orbitofrontal activity predicts better memory for faces." Eur J Neurosci 17(12): 2755-2758.

Frey S, Kostopoulos P, Petrides M (2004): "Orbitofrontal contribution to auditory encoding." Neuroimage 22(3): 1384-1389.

Fuster JM: "The Prefrontal Cortex: Anatomy, Physiology, ans Neuropsychology of the Frontal Lobe." Raven Press, New York 1980.

Goldman AL, Pezawas L, Mattay VS, Fischl B, Verchinski BA, Zoltick B, Weinberger DR, Meyer-Lindenberg A (2008): "Heritability of brain morphology related to schizophrenia: a large-scale automated magnetic resonance imaging segmentation study." Biol Psychiatry 63(5): 475-483.

Gur RC, Mozley PD, Resnick SM, Gottlieb GL, Kohn M, Zimmerman R, Herman G, Atlas S, Grossman R, Berretta D, et al. (1991): "Gender differences in age effect on brain atrophy measured by magnetic resonance imaging." Proc Natl Acad Sci U S A 88 (7): 2845-2849.

Gur RE, Cowell PE, Latshaw A, Turetsky BI, Grossman RI, Arnold SE, Bilker WB, Gur RC (2000): "Reduced dorsal and orbital prefrontal gray matter volumes in schizophrenia." Arch Gen Psychiatry 57(8): 761-768.

Gurling HM, Critchley H, Datta SR, McQuillin A, Blaveri E, Thirumalai S, Pimm J, Krasucki R, Kalsi G, Quested D, et al. (2006): "Genetic association and brain morphology studies and the chromosome 8p22 pericentriolar material 1 (PCM1) gene in susceptibility to schizophrenia." Arch Gen Psychiatry 63(8): 844-854.

Harms MP, Wang L, Campanella C, Aldridge K, Moffitt AJ, Kuelper J, Ratnanather JT, Miller MI, Barch DM, Csernansky JG (2010): "Structural abnormalities in gyri of the prefrontal cortex in individuals with schizophrenia and their unaffected siblings." Br J Psychiatry 196(2): 150-157. 
Hoptman MJ, Volavka J, Weiss EM, Czobor P, Szeszko PR, Gerig G, Chakos M, Blocher J, Citrome LL, Lindenmayer JP, et al. (2005): "Quantitative MRI measures of orbitofrontal cortex in patients with chronic schizophrenia or schizoaffective disorder." Psychiatry Res 140(2): 133-145.

Hornak J, Rolls ET, Wade D (1996): "Face and voice expression identification in patients with emotional and behavioural changes following ventral frontal lobe damage." Neuropsychologia 34(4): 247-261.

Hornak J, Bramham J, Rolls ET, Morris RG, O'Doherty J, Bullock PR, Polkey CE (2003): "Changes in emotion after circumscribed surgical lesions of the orbitofrontal and cingulate cortices." Brain 126(Pt 7): 1691-1712.

Hulshoff Pol HE, Schnack HG, Mandl RC, Brans RG, van Haren NE, Baare WF, van Oel CJ, Collins DL, Evans AC, Kahn RS (2006): "Gray and white matter density changes in monozygotic and same-sex dizygotic twins discordant for schizophrenia using voxel-based morphometry." Neuroimage 31(2): 482-488.

Ilonen T, Taiminen T, Lauerma H, Karlsson H, Helenius HY, Tuimala P, Leinonen KM, Wallenius E, Salokangas RK (2000): "Impaired Wisconsin card sorting test performance in first-episode schizophrenia: resource or motivation deficit?" Compr Psychiatry 41(5): 385-391.

Ingvar DH, Franzen G (1974): "Abnormalities of cerebral blood flow distribution in

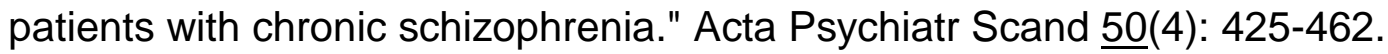

Kalayam B, Alexopoulos GS (1999): "Prefrontal dysfunction and treatment response in geriatric depression." Arch Gen Psychiatry $\underline{56}(8)$ : 713-718.

Karno M, Golding JM, Sorenson SB, Burnam MA (1988): "The epidemiology of obsessive-compulsive disorder in five US communities." Arch Gen Psychiatry 45(12): 1094-1099.

Kay SR, Fiszbein A, Opler LA (1987): "The positive and negative syndrome scale (PANSS) for schizophrenia." Schizophr Bull 13(2): 261-276. 
Kessler RC, Rubinow DR, Holmes C, Abelson JM, Zhao S (1997): "The epidemiology of DSM-III-R bipolar I disorder in a general population survey." Psychol Med 27(5): 1079-1089.

Kim JJ, Lee MC, Kim J, Kim IY, Kim SI, Han MH, Chang KH, Kwon JS (2001): "Grey matter abnormalities in obsessive-compulsive disorder: statistical parametric mapping of segmented magnetic resonance images." $\mathrm{Br} \mathrm{J}$ Psychiatry 179: 330-334.

Kringelbach ML (2005): "The human orbitofrontal cortex: linking reward to hedonic experience." Nat Rev Neurosci $\underline{6}(9)$ : 691-702.

Kringelbach ML, Rolls ET (2004): "The functional neuroanatomy of the human orbitofrontal cortex: evidence from neuroimaging and neuropsychology." Prog Neurobiol 72(5): 341-372.

Kwon JS, Kim JJ, Lee DW, Lee JS, Lee DS, Kim MS, Lyoo IK, Cho MJ, Lee MC (2003): "Neural correlates of clinical symptoms and cognitive dysfunctions in obsessive-compulsive disorder." Psychiatry Res 122(1): 37-47.

Lacerda AL, Hardan AY, Yorbik O, Vemulapalli M, Prasad KM, Keshavan MS (2007): "Morphology of the orbitofrontal cortex in first-episode schizophrenia: relationship with negative symptomatology." Prog Neuropsychopharmacol Biol Psychiatry 31(2): 510-516.

Liu X, Matochik JA, Cadet JL, London ED (1998): "Smaller volume of prefrontal lobe in polysubstance abusers: a magnetic resonance imaging study." Neuropsychopharmacology 18(4): 243-252.

Lopez-Larson MP, DelBello MP, Zimmerman ME, Schwiers ML, Strakowski SM (2002): "Regional prefrontal gray and white matter abnormalities in bipolar disorder." Biol Psychiatry 52(2): 93-100.

Manji HK, Moore GJ, Chen G (2000): "Clinical and preclinical evidence for the neurotrophic effects of mood stabilizers: implications for the pathophysiology and treatment of manic-depressive illness." Biol Psychiatry 48(8): 740-754. 
Mesulam MM, Mufson EJ (1984): "Neural inputs into the nucleus basalis of the substantia innominata (Ch4) in the rhesus monkey." Brain 107 (Pt 1): 253274.

Mitz AR, Godschalk M (1989): "Eye-movement representation in the frontal lobe of rhesus monkeys." Neurosci Lett 106(1-2): 157-162.

Molina V, Sanz J, Benito C, Palomo T (2004): "Direct association between orbitofrontal atrophy and the response of psychotic symptoms to olanzapine in schizophrenia." Int Clin Psychopharmacol 19(4): 221-228.

Molina V, Reig S, Sanz J, Palomo T, Benito C, Sanchez J, Pascau J, Desco M (2007): "Changes in cortical volume with olanzapine in chronic schizophrenia." Pharmacopsychiatry $\underline{40}(4)$ : 135-139.

Montgomery SA, Asberg M (1979): "A new depression scale designed to be sensitive to change." Br J Psychiatry 134: 382-389.

Moore GJ, Bebchuk JM, Wilds IB, Chen G, Manji HK (2000): "Lithium-induced increase in human brain grey matter." Lancet 356(9237): 1241-1242.

Mora A, Sabio G, Gonzalez-Polo RA, Cuenda A, Alessi DR, Alonso JC, Fuentes JM, Soler G, Centeno F (2001): "Lithium inhibits caspase 3 activation and dephosphorylation of PKB and GSK3 induced by $\mathrm{K}+$ deprivation in cerebellar granule cells." J Neurochem 78(1): 199-206.

Morecraft RJ, Geula C, Mesulam MM (1992): "Cytoarchitecture and neural afferents of orbitofrontal cortex in the brain of the monkey." J Comp Neurol 323(3): 341-358.

Nakamura M, Nestor PG, Levitt JJ, Cohen AS, Kawashima T, Shenton ME, McCarley RW (2008): "Orbitofrontal volume deficit in schizophrenia and thought disorder." Brain 131(Pt 1): 180-195.

Nery FG, Chen HH, Hatch JP, Nicoletti MA, Brambilla P, Sassi RB, Mallinger AG, Keshavan MS,Soares JC (2009): "Orbitofrontal cortex gray matter volumes 
in bipolar disorder patients: a region-of-interest MRI study." Bipolar Disord 11(2): 145-153.

Nolan CL, Moore GJ, Madden R, Farchione T, Bartoi M, Lorch E, Stewart CM, Rosenberg DR (2002): "Prefrontal cortical volume in childhood-onset major depression: preliminary findings." Arch Gen Psychiatry 59 (2): 173-179.

Oldfield RC (1971): "The assessment and analysis of handedness: the Edinburgh inventory." Neuropsychologia $\underline{9}(1)$ : 97-113.

Ongur D, Price JL (2000): "The organization of networks within the orbital and medial prefrontal cortex of rats, monkeys and humans." Cereb Cortex $\underline{10}(3)$ : 206-219.

Ongur D, An X, Price JL (1998): "Prefrontal cortical projections to the hypothalamus in macaque monkeys." J Comp Neurol 401(4): 480-505.

Pandya DN, Van Hoesen GW, Mesulam MM (1981): "Efferent connections of the cingulate gyrus in the rhesus monkey." Exp Brain Res 42(3-4): 319-330.

Pantelis C, Velakoulis D, McGorry PD, Wood SJ, Suckling J, Phillips LJ, Yung AR, Bullmore ET, Brewer W, Soulsby B, et al. (2003): "Neuroanatomical abnormalities before and after onset of psychosis: a cross-sectional and longitudinal MRI comparison." Lancet 361(9354): 281-288.

Peterson BS, Staib L, Scahill L, Zhang H, Anderson C, Leckman JF, Cohen DJ, Gore JC, Albert J, Webster R (2001): "Regional brain and ventricular volumes in Tourette syndrome." Arch Gen Psychiatry 58(5): 427-440.

Petrides M, Pandya DN (2002): "Comparative cytoarchitectonic analysis of the human and the macaque ventrolateral prefrontal cortex and corticocortical connection patterns in the monkey." Eur J Neurosci 16(2): 291-310.

Pritchard TC, Hamilton RB, Morse JR, Norgren R (1986): "Projections of thalamic gustatory and lingual areas in the monkey, Macaca fascicularis." J Comp Neurol 244(2): 213-228. 
Raine A, Lencz T, Bihrle S, LaCasse L, Colletti P (2000): "Reduced prefrontal gray matter volume and reduced autonomic activity in antisocial personality

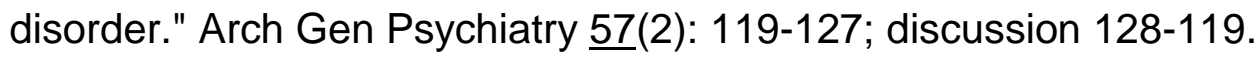

Ray JP, Price JL (1993): "The organization of projections from the mediodorsal nucleus of the thalamus to orbital and medial prefrontal cortex in macaque monkeys." J Comp Neurol 337(1): 1-31.

Rempel-Clower NL, Barbas H (1998): "Topographic organization of connections between the hypothalamus and prefrontal cortex in the rhesus monkey." J Comp Neurol 398(3): 393-419.

Rico B, Cavada C (1998): "Adrenergic innervation of the monkey thalamus: an immunohistochemical study." Neuroscience 84(3): 839-847.

Rolls ET (2004): "The functions of the orbitofrontal cortex." Brain Cogn 55(1): 1129.

Rolls ET, Baylis LL (1994): "Gustatory, olfactory, and visual convergence within the primate orbitofrontal cortex." J Neurosci 14(9): 5437-5452.

Rolls ET, Yaxley S, Sienkiewicz ZJ (1990): "Gustatory responses of single neurons in the caudolateral orbitofrontal cortex of the macaque monkey." $\mathrm{J}$ Neurophysiol 64(4): 1055-1066.

Rolls ET, Hornak J, Wade D, McGrath J (1994): "Emotion-related learning in patients with social and emotional changes associated with frontal lobe damage." J Neurol Neurosurg Psychiatry 57(12): 1518-1524.

Rolls ET, Critchley HD, Treves A (1996): "Representation of olfactory information in the primate orbitofrontal cortex." J Neurophysiol 75(5): 1982-1996.

Rolls ET, O'Doherty J, Kringelbach ML, Francis S, Bowtell R, McGlone F (2003): "Representations of pleasant and painful touch in the human orbitofrontal and cingulate cortices." Cereb Cortex 13(3): 308-317. 
Rotge JY, Guehl D, Dilharreguy B, Tignol J, Bioulac B, Allard M, Burbaud P, Aouizerate B (2009): "Meta-analysis of brain volume changes in obsessivecompulsive disorder." Biol Psychiatry 65(1): 75-83.

Rotge JY, Guehl D, Dilharreguy B, Cuny E, Tignol J, Bioulac B, Allard M, Burbaud P, Aouizerate B (2008): "Provocation of obsessive-compulsive symptoms: a quantitative voxel-based meta-analysis of functional neuroimaging studies." J Psychiatry Neurosci 33(5): 405-412.

Roth RM, Saykin AJ, Flashman LA, Pixley HS, West JD, Mamourian AC (2007): "Event-related functional magnetic resonance imaging of response inhibition in obsessive-compulsive disorder." Biol Psychiatry 62(8): 901-909.

Saß, Wittchen Zaudig, Eds: "Diagnostisches und Statistisches Manual Psychiatrischer Störungen DSM-IV" Hogrefe, Göttingen 1996.

Saxena S, Brody AL, Maidment KM, Dunkin JJ, Colgan M, Alborzian S, Phelps ME Baxter LR, Jr. (1999): "Localized orbitofrontal and subcortical metabolic changes and predictors of response to paroxetine treatment in obsessivecompulsive disorder." Neuropsychopharmacology 21(6): 683-693.

Scherk H, Falkai P (2006): "Effects of antipsychotics on brain structure." Curr Opin Psychiatry 19(2): 145-150.

Selemon LD, Goldman-Rakic PS (1999): "The reduced neuropil hypothesis: a circuit based model of schizophrenia." Biol Psychiatry 45(1): 17-25.

Small DM, Zald DH, Jones-Gotman M, Zatorre RJ, Pardo JV, Frey S, Petrides M (1999): "Human cortical gustatory areas: a review of functional neuroimaging data." Neuroreport 10(1): 7-14.

Small DM, Bender G, Veldhuizen MG, Rudenga K, Nachtigal D, Felsted J (2007): "The role of the human orbitofrontal cortex in taste and flavor processing." Ann N Y Acad Sci 1121: 136-151.

Smieskova R, Fusar-Poli P, Allen P, Bendfeldt K, Stieglitz RD, Drewe J, Radue EW, McGuire PK, Riecher-Rossler A, Borgwardt SJ (2009): "The effects of 
antipsychotics on the brain: what have we learnt from structural imaging of schizophrenia?--a systematic review." Curr Pharm Des 15(22): 2535-2549.

Spironelli C, Angrilli A, Stegagno L (2008): "Failure of language lateralization in schizophrenia patients: an ERP study on early linguistic components." J Psychiatry Neurosci 33(3): 235-243.

Suzuki WA, Amaral DG (1994): "Perirhinal and parahippocampal cortices of the macaque monkey: cortical afferents." J Comp Neurol 350(4): 497-533.

Toro CT, Hallak JE, Dunham JS, Deakin JF (2006): "Glial fibrillary acidic protein and glutamine synthetase in subregions of prefrontal cortex in schizophrenia and mood disorder." Neurosci Lett 404(3): 276-281.

Walker A (1940): "A cytoarchitectural study of the prefrontal area of the macaque monkey." J Comp Neurol (73): 59-86.

Wang HD, Dunnavant FD, Jarman T, Deutch AY (2004): "Effects of antipsychotic drugs on neurogenesis in the forebrain of the adult rat." Neuropsychopharmacology 29(7): 1230-1238.

Woods SW (2003): "Chlorpromazine equivalent doses for the newer atypical antipsychotics." J Clin Psychiatry 64(6): 663-667.

Young RC, Biggs JT, Ziegler VE, Meyer DA (1978): "A rating scale for mania: reliability, validity and sensitivity." Br J Psychiatry 133: 429-435.

Zatorre RJ, Jones-Gotman M, Evans AC, Meyer E (1992): "Functional localization and lateralization of human olfactory cortex." Nature $\underline{360}$ (6402): 339-340. 


\section{$9 \quad$ Anhang}

\subsection{PANSS}

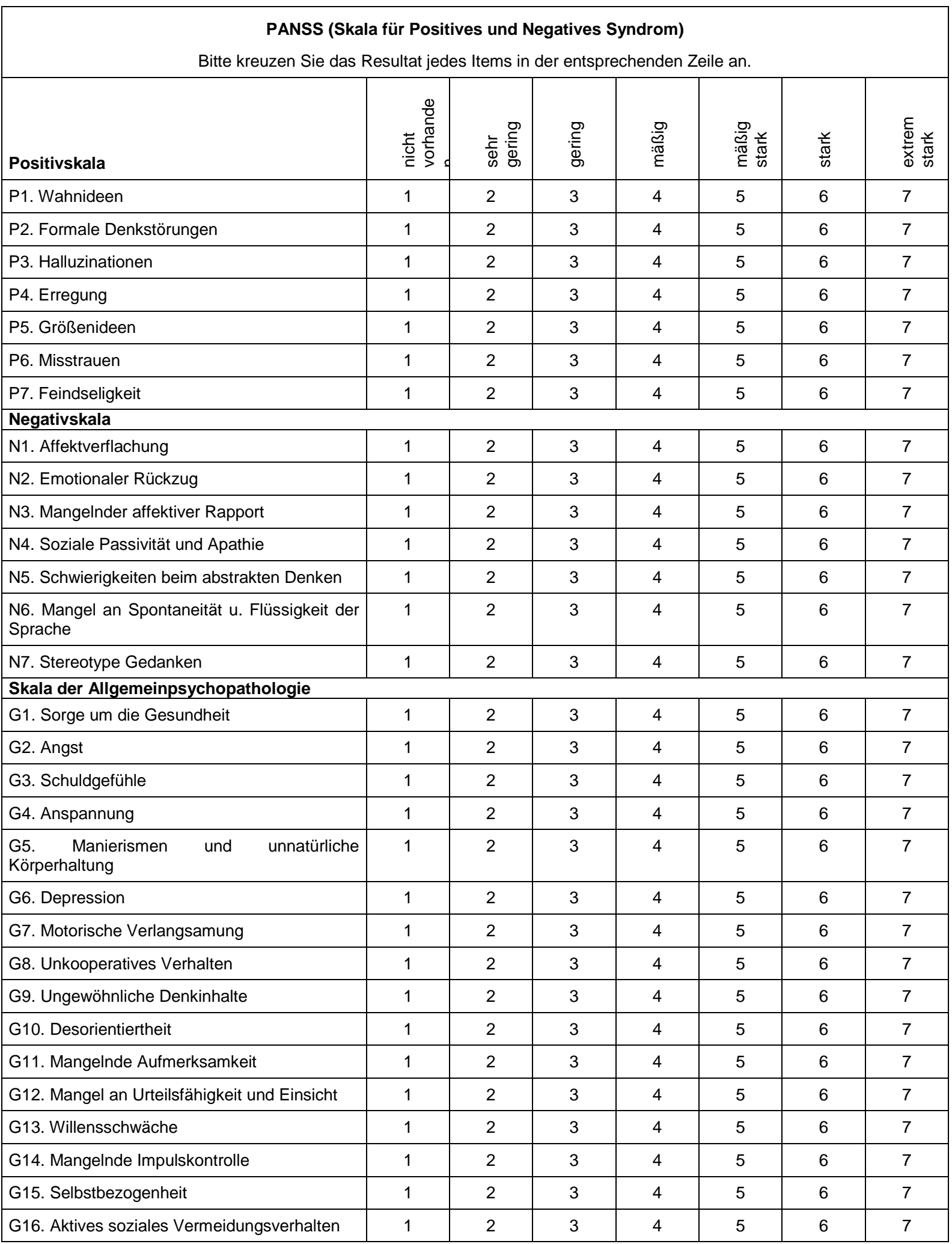




\section{$9.2 \quad$ MADRS}

\section{Anleitung: Bitte für jede Feststellung nur 1 Ziffer ankreuzen!}

\begin{tabular}{|c|c|c|c|}
\hline \multirow{2}{*}{\multicolumn{2}{|c|}{ 1. Sichtbare Traurigkeit }} & 0 & Keine Traurigkeit \\
\hline & & 1 & \\
\hline \multicolumn{2}{|r|}{ (mehr als eine gewöhnliche, vorübergehende Niedergeschla- } & 2 & Schaut niedergeschlagen aus, aber heitert ohne Mühe auf \\
\hline \multicolumn{2}{|r|}{ genheit), die sich in Sprache, Gesichtsausdruck und Körper- } & 3 & \\
\hline \multicolumn{2}{|r|}{ haltung äußern. } & 4 & Erscheint meistens traurig und unglücklich. \\
\hline \multirow{2}{*}{\multicolumn{2}{|c|}{ Einzuschätzen nach der Fähigkeit aufzuheitern. }} & 5 & \\
\hline & & 6 & Schaut immer unglücklich aus; höchst verzweifelt. \\
\hline \multirow[t]{9}{*}{2.} & \multirow{9}{*}{$\begin{array}{l}\text { Mitgeteilte Traurigkeit } \\
\text { Steht für Mitteilungen über depress. Erleben, ungeachtet } \\
\text { des äußeren Erscheinungsbildes. Schließt Niedergeschla- } \\
\text { genheit, Verzweiflung und das Gefühl ein, dass es weder } \\
\text { Hoffnung noch Hilfe geben kann. } \\
\text { Einzuschätzen danach, in welcher Intensität und Dauer und } \\
\text { in welchem Ausmaß äußere Ereignisse nach Berichten des } \\
\text { Patienten die Stimmung beeinflussen können. }\end{array}$} & 0 & Den Umständen entsprechende, gelegentliche Traurigkeit. \\
\hline & & 1 & \\
\hline & & 2 & Traurig oder bedrückt, aber heitert ohne Mühe auf. \\
\hline & & 3 & \\
\hline & & 4 & \multirow{2}{*}{$\begin{array}{l}\text { Beherrschendes Gefühl von Traurigkeit und Schwermut; } \\
\text { Gemütslage wird immer noch von äußeren Umständen } \\
\text { beeinflusst. }\end{array}$} \\
\hline & & & \\
\hline & & 5 & \\
\hline & & 6 & \multirow{2}{*}{$\begin{array}{l}\text { Anhaltende und unveränderte Traurigkeit, Unglücklichsein } \\
\text { und Verzweiflung. }\end{array}$} \\
\hline & & & \\
\hline \multirow[t]{9}{*}{3.} & \multirow{9}{*}{$\begin{array}{l}\text { Innere Anspannung } \\
\text { Steht für Gefühle schwer definierbaren Unwohlseins, } \\
\text { Gereiztheit, innerer Unruhe, psychischer Anspannungen } \\
\text { die bis zu Panik, Schrecken und unerträglichen Quelen } \\
\text { reichen können. }\end{array}$} & 0 & Gelassen, nur flüchtige innere Spannungen. \\
\hline & & 1 & \\
\hline & & 2 & Gelegentliches Gereiztsein und schwer definierbares Unwohlsein. \\
\hline & & 3 & \\
\hline & & 4 & \multirow{2}{*}{$\begin{array}{l}\text { Anhaltendes inneres Spannungsgefühl oder zeitweilig auftretende } \\
\text { Panik, die der Patient nur mit beträchtlicher Mühe meistern kann. }\end{array}$} \\
\hline & & & \\
\hline & & 5 & \\
\hline & & 6 & \multirow{2}{*}{$\begin{array}{l}\text { Nicht nachlassende, unerträgliche Furcht oder Qualen. } \\
\text { Überwältigende Panik }\end{array}$} \\
\hline & & & \\
\hline \multirow{8}{*}{ 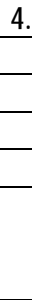 } & Reduzierter Schlaf & 0 & Normaler Schlaf \\
\hline & Steht für das Erleben einer reduzierten Schlafdauer oder & 1 & \\
\hline & \multirow{6}{*}{$\begin{array}{l}\text { Schlaftiefe, vergleichen mit dem normalen Schlaf- } \\
\text { erhalten des Patienten in gesunden Zeiten. }\end{array}$} & 2 & Geringfügige Schwierigkeit einzuschlafen oder etwas reduzierter, \\
\hline & & & leichter oder unregelmäßiger Schlaf. \\
\hline & & 3 & \\
\hline & & 4 & Reduzierter oder mindestens 2 Stunden lang unterbrochener Schlaf. \\
\hline & & 5 & \\
\hline & & 6 & Weniger als 2 oder 3 Stunden Schlaf \\
\hline 5. & Reduzierter Appetit & 0 & Normaler oder größerer Appetit. \\
\hline & Steht für das Gefühl, keinen Appetit zu haben, verglichen mit & 1 & \\
\hline & dem gesunden Zustand. & 2 & Etwas reduziert \\
\hline & Einzustufen nach dem Verlust des Verlangens nach Essen & 3 & \\
\hline & oder der Notwendigkeit, sich zum Essen zu zwingen. & 4 & Kein Appetit: Essen schmeckt nicht. \\
\hline & & 5 & \\
\hline & & 6 & Muss zum Essen überredet werden. \\
\hline 6. & Konzentrationsschwierigkeiten & 0 & Keine Schwierigkeiten beim Konzentrieren. \\
\hline & Steht für Schwierigkeiten, seine Gedanken zu sammeln, bis & 1 & \\
\hline & hin zum vollständigen Verlust der Konzentrationsfähigkeit. & 2 & Hat manchmal Schwierigkeiten, die Gedanken zu sammeln. \\
\hline & Einzustufen nach Intensität, Häufigkeit und Ausmaß der & 3 & \\
\hline & Konzentrationsstörung. & 4 & Schwierigkeiten, sich zu konzentrieren und seine Gedanken zu \\
\hline & & & sammeln, so dass die Fähigkeit, zu lesen und einem Gespräch zu \\
\hline & & & folgen, eingeschränkt ist. \\
\hline & & 5 & \\
\hline & & 6 & Kann nur mit größter Mühe lesen oder sich an einem Gespräch \\
\hline & & & beteiligen. \\
\hline 7. & Antriebsmangel & 0 & Keine Schwierigkeiten, in Gang zu kommen. Keine Trägheit. \\
\hline & Steht für die Schwierigkeit, in Gang zu kommen, oder für die & 1 & \\
\hline & Langsamkeit, mit den täglichen Aktivitäten anzufangen und & 2 & Schwierigkeiten, mit Aktivitäten anzufangen. \\
\hline & sie durchzuführen. & 3 & \\
\hline
\end{tabular}




\begin{tabular}{|c|c|c|}
\hline & 4 & Schwierigkeiten, mit einfachen Routinetätigkeiten zu beginnen; \\
\hline & & sie können nur mit Mühle ausgeführt werden. \\
\hline & 5 & \\
\hline & 6 & Völlige Antriebslosigkeit, Unfähigkeit, etwas ohne Hilfe zu tun. \\
\hline Gefühl der Gefühllosigkeit & 0 & Normales Interesse an Umwelt und anderen Menschen \\
\hline Steht für das subjektive Erleben von vermindertem Interesse & 1 & \\
\hline an der Umwelt oder an Aktivitäten, die sonst immer Spaß & 2 & Eingeschränktes Vermögen, an den gewohnten Interessen Freude \\
\hline gemacht haben. Die Fähigkeit, auf Situationen oder Menschen & & zu finden. \\
\hline emotional adäquat zu reagieren, ist eingeschränkt. & 3 & \\
\hline & 4 & Verlust des Interesses an der Umwelt, Verlust der Gefühle für \\
\hline & & Freunde und Bekannte. \\
\hline & 5 & \\
\hline & 6 & Das Erleben, emotional gelähmt zu sein, Unfähigkeit, Ärger, Trauer \\
\hline & & oder Freunde zu empfinden; völliges, evtl. sogar sogar schmerz- \\
\hline & & liches Unvermögen, etwas für nahe Verwandte oder Freunde zu \\
\hline & & empfinden. \\
\hline 9. $\quad$ Pessimistische Gedanken & 0 & Keine pessimistischen Gedanken \\
\hline Steht für Gedanken über Schuld, Mnderwertigkeit, Selbst- & 1 & \\
\hline vorwürfe, Versündigung, Reue und Ruin. & 2 & Fluktuierende Ideen über Versagen, Selbstverachtung und Selbst- \\
\hline & & vorwürfe. \\
\hline & 3 & \\
\hline & 4 & Dauernde Selbstanklagen oder bestimmte, aber immer noch \\
\hline & & rationale Vorstellungen über Schuld und Sühne. Anwachsender \\
\hline & & Pessimismus über die Zukunft. \\
\hline & 5 & \\
\hline & 6 & Wahnvorstellungen über Ruin, Reue und unverzeihliche Sünden: \\
\hline & & absurde und unerschütterliche Selbstbezichtigungen. \\
\hline 10. Suizidgedanken & 0 & Genießt das Leben oder nimmt es, wie es kommt. \\
\hline Steht für das Gefühl, dass das Leben nicht der Mühe wert ist, & 1 & \\
\hline dass ein natürlicher Tod willkommen wäre: Suizidgedanken & 2 & Lebensmüde. Nur flüchtige Selbstmordgedanken. \\
\hline und Vorbereitungen zum Selbstmord. Suizidversuche an sich & 3 & \\
\hline sollten die Bewertung nicht beeinflussen. & 4 & Wahrscheinlich wäre es besser, tot zu sein. Suizidgedanken \\
\hline & & sind häufig; Selbstmord wird als mögliche Lösung betrachtet, \\
\hline & & aber ohne konkrete Pläne oder Absichten. \\
\hline & 5 & \\
\hline & 6 & Konkrete Selbstmordpläne für den Fall, dass sich eine Gelegen- \\
\hline & & heit bietet. Aktive Vorbereitungen zum Suizid. \\
\hline $\begin{array}{l}\text { Bitte prüfen Sie, ob Sie alle erforderlichen Angaben } \\
\text { gemacht bzw. die jeweiligen Ziffern zutreffend } \\
\text { angekreuzt haben! }\end{array}$ & & \\
\hline
\end{tabular}




\subsection{CGI (Clinical Global Impressions)}

Ziehen Sie Ihren gesamten Erfahrungsschatz an dieser Art von Krankheit in Betracht, und geben Sie an, wie hoch Sie den jetzigen Grad der seelischen Erkrankungen des Patienten einschätzen.

$0 \quad$ Nicht zu beurteilen

1 Patient ist überhaupt nicht krank

2 Patient ist ein Grenzfall psychiatrischer Behandlung

$3 \quad$ Patient ist nur leicht krank

$4 \quad$ Patient ist mäßig krank

$5 \quad$ Patient ist deutlich krank

$6 \quad$ Patient ist schwer krank

7 Patient gehört zu den extrem schwer Kranken 


\subsection{GAF (Global Assessment of Functioning)}

Beurteile GAF-Score anhand der folgenden Beschreibungen für die letzte Woche

BEACHTE: Man bewerte die niedrigste Stufe der psychischen und sozialen Leistungsfähigkeit des Patienten in der letzten zurückliegenden Woche. Dabei wird die niedrigste zutreffende Bewertungsstufe der Leistungsfähigkeit aus einem hypothetischen Kontinuum des psychischen Krankseins ausgewählt, wenn z. B. das Verhalten des Patienten erheblich von Wahnvorstellungen beeinflusst wird (Stufe 21-30) so bewertet man dies mit einem Wert, der in dieser Bewertungsstufe liegt und zwar auch dann, wenn erhebliche Beeinträchtigungen auf verschiedenen Gebieten (Stufe 31-40) vorhanden sind; wenn nötig, so verwende man Zwischenstufen (z. B. 35, 58, 63).

\begin{tabular}{|c|c|}
\hline $100-91$ & $\begin{array}{l}\text { Hervorragende Leistungsfähigkeit in einem breiten Spektrum von Aktivitäten; } \\
\text { Schwierigkeiten im Leben scheinen nie außer Kontrolle zu geraten; wird von anderen } \\
\text { wegen einer Vielzahl positiver Qualitäten geschätzt. Der Patient hat keinerlei } \\
\text { Symptome. }\end{array}$ \\
\hline $90-81$ & $\begin{array}{l}\text { Keine oder nur minimale Symptome; gute Leistungsfähigkeit in allen Gebieten; } \\
\text { interessiert sich vielseitig; entwickelt viele Aktivitäten; gute soziale Anpassung; im } \\
\text { allgemeinen zufrieden mit dem Leben, übliche Alltagsprobleme oder -sorgen. }\end{array}$ \\
\hline $80-71$ & $\begin{array}{l}\text { Wenn Symptome vorliegen, sind diese vorübergehende oder zu erwartenden } \\
\text { Reaktionen auf psychosoziale Belastungsfaktoren (z. B. Konzentrationsstörungen nach } \\
\text { einem Familienstreit); höchstens leichte Beeinträchtigung der sozialen, beruflichen und } \\
\text { schulischen Leistungsfähigkeit (z. B. zeitweises Zurückbleiben in der Schule). }\end{array}$ \\
\hline $70-61$ & $\begin{array}{l}\text { Einige leichte Symptome sind vorhanden (z.B. depressive Stimmung, leichte } \\
\text { Schlaflosigkeit); oder es gibt Schwierigkeiten in den verschiedenen Leistungsbereichen, } \\
\text { aber im allgemeinen ist jedoch die Leistungsfähigkeit recht gut; einige bedeutsame } \\
\text { zwischenmenschliche Beziehungen werden aufrecht erhalten (die meisten Laien } \\
\text { würden den Patienten nicht als krank ansehen) }\end{array}$ \\
\hline $60-51$ & $\begin{array}{l}\text { Mäßig ausgeprägte Symptome (z. B. Affektverflachung, weitschweifige Sprache, } \\
\text { gelegentliche Panikattacken) sind vorhanden, oder es besteht eine mäßig } \\
\text { eingeschränkte Leistungsfähigkeit (z.B. wenige Freunde, Konflikte mit Arbeitskollegen } \\
\text { oder Bezugspersonen). }\end{array}$ \\
\hline $50-41$ & $\begin{array}{l}\text { Ernsthafte Symptome (z. B. Suizidgedanken, schwere Zwangsrituale, häufige } \\
\text { Ladendiebstähle) oder starke Leistungseinbußen bzw. starke Beeinträchtigung der sozialen, } \\
\text { beruflichen oder schulischen Leistungsfähigkeit (z. B. keine Freunde, Unfähigkeit, eine } \\
\text { Arbeitsstelle zu behalten) }\end{array}$ \\
\hline $40-31$ & $\begin{array}{l}\text { Schwere Beeinträchtigung auf verschiedenen Gebieten (vor allem Arbeit, familiäre } \\
\text { Beziehungen, Urteilsvermögen, Denken, Stimmung; z.B. Meiden der Freunde; } \\
\text { Vernachlässigung der Familie; nicht mehr in der Lage, den Haushalt zu bewältigen; gestörte } \\
\text { Realitätseinschätzung; gestörtes Kommunikationsverhalten manchmal unverständliche, } \\
\text { unlogische oder irrelevante Sprachgestaltung; oder ernsthafter Suizidversuch). }\end{array}$ \\
\hline $30-21$ & $\begin{array}{l}\text { Leistungsunfähigkeit in fast allen Gebieten (z.B. bleibt täglich im Bett); oder das Verhalten } \\
\text { ist erheblich beeinflusst durch Wahnvorstellungen und Halluzinationen; oder schwere } \\
\text { Beeinträchtigung der Kommunikation (z.B. manchmal im Denken inkohärent oder nicht } \\
\text { ansprechbar) oder des Urteilsvermögens (z.B. grob auffällige unangemessene } \\
\text { Handlungen). }\end{array}$ \\
\hline $20-11$ & $\begin{array}{l}\text { Benötigt irgendeine Art der Überwachung (Selbst- und Fremdgefährdung), um nicht sich } \\
\text { selbst oder andere zu verletzen, oder um eine minimale persönliche Hygiene } \\
\text { aufrechtzuerhalten (z.B. wiederholte Suizidversuche sind vorgekommen); wird häufig } \\
\text { gewalttätig; eine manische Erregung ist vorhanden; verschmiert die Faeces; oder massive } \\
\text { Beeinträchtigung der Kommunikationsfähigkeit (durchgehend inkohärent oder mutistisch). }\end{array}$ \\
\hline $10-1$ & $\begin{array}{l}\text { Braucht ständig Überwachung für mehrere Tage, um sich oder andere nicht zu verletzen; oder } \\
\text { macht keine Anstalten, eine minimale körperliche Hygiene aufrecht zu erhalten (es ist z.B. eine } \\
\text { intensive Pflege und eine besondere Überwachung durch das Personal notwendig); oder } \\
\text { ernsthafter Selbstmordversuch mit eindeutiger Tötungsabsicht. }\end{array}$ \\
\hline
\end{tabular}




\section{Danksagung}

An dieser Stelle möchte ich mich herzlichst bei Herrn Prof. Dr. med. Falkai (Direktor der Abteilung Psychiatrie und Psychotherapie, Universitätsklinikum Georg-August-Universität Göttingen) für die Überlassung des interessanten Themas bedanken.

Meinem wissenschaftlichen Betreuer, Herrn PD Dr. med. Thomas Wobrock (Abteilung Psychiatrie und Psychotherapie, Universitätsklinikum Georg-AugustUniversität Göttingen) sei für sein großes Interesse und die vielen praktischen Hinweise im Rahmen der Studie und des Schreibens der Arbeit, sowie für die intensive Betreuung in jeder Phase des Entstehens dieser Dissertation an dieser Stelle ganz besonderer Dank gesagt. 


\section{Lebenslauf}

Am 17.03.1984 wurde ich als drittes Kind des Oberstudienrates Karl- Wilhelm Meyer und der Lehrerin Margrit Bertram- Meyer in Hannover geboren.

In Pattensen bei Hannover verbrachte ich meine Kindheit und besuchte von 1990 bis 1996 die dortige Grundschule sowie Orientierungsstufe. Nachfolgend besuchte ich das Humboldt- Gymnasium in Hannover und beendete meine schulische Laufbahn mit dem Abitur im Jahr 2003. Anschließend folgte ein halbjähriger Auslandsaufenthalt in Sydney, Australien.

Zum Sommersemester 2004 begann ich mein Studium der Humanmedizin an der Georg- August- Universität in Göttingen. Im Frühjahr 2006 legte ich den ersten Teil der ärztlichen Gesamtprüfung ab. Während meines klinischen Studiums absolvierte ich Famulaturen und Praktika innerhalb verschiedenster Bereiche der Humanmedizin in Göttingen, Hannover, Vancouver/ Kanada sowie Galway /Irland.

Studienbegleitend arbeitete ich als Tutorin des makroskopischen Kursus der Anatomie innerhalb des Zentrums Anatomie der Georg- August- Universität.

Im Sommer 2007 begann ich meine Dissertationsarbeit unter der Leitung von Herrn Prof. Dr. med. Falkai, Direktor der Abteilung Psychiatrie und Psychotherapie der Universitätsklinikums der Georg-August-Universität Göttingen, und unter der Betreuung von Priv.-Doz. Dr. med. Thomas Wobrock.

Im Februar 2009 folgte das praktische Jahr mit Tertialen in Zürich (Chirurgie) und Göttingen (Pädiatrie und Innere Medizin).

Im Mai 2010 habe ich mein Studium der Humanmedizin mit dem zweiten Teil der ärztlichen Gesamtprüfung erfolgreich abgeschlossen und am 27.05.2010 die ärztliche Approbation erteilt bekommen. 
